\title{
The quark-gluon vertex in Landau gauge QCD: Its role in dynamical chiral symmetry breaking and quark confinement
}

\author{
Reinhard Alkofer ${ }^{\mathrm{a}}$, Christian S. Fischer ${ }^{\mathrm{b}, *}$, Felipe J. Llanes-Estrada ${ }^{\mathrm{c}}$, \\ Kai Schwenzer ${ }^{\mathrm{a}}$ \\ a Institut für Physik der Karl-Franzens Universität Graz, A-8010 Graz, Austria \\ ${ }^{\mathrm{b}}$ Institut für Kernphysik der Technische Universität Darmstadt, Schlossgartenstrasse 9, D-64289 Darmstadt, Germany \\ ${ }^{\mathrm{C}}$ Departamento de Física Teórica I de la Universidad Complutense, 28040 Madrid, Spain
}

\section{A R T I C L E I N F O}

\section{Article history:}

Received 21 April 2008

Accepted 3 July 2008

Available online 19 July 2008

\section{PACS:}

11.10.St

11.15. $-\mathrm{q}$

11.30.Rd

12.38.Aw

Keywords:

Non-perturbative QCD

Confinement

Dynamical chiral symmetry breaking

Quark-gluon vertex

Dyson-Schwinger equations

\begin{abstract}
A B S T R A C T
The infrared behavior of the quark-gluon vertex of quenched Landau gauge QCD is studied by analyzing its Dyson-Schwinger equation. Building on previously obtained results for Green functions in the Yang-Mills sector, we analytically derive the existence of powerlaw infrared singularities for this vertex. We establish that dynamical chiral symmetry breaking leads to the self-consistent generation of components of the quark-gluon vertex forbidden when chiral symmetry is forced to stay in the Wigner-Weyl mode. In the latter case the running strong coupling assumes an infrared fixed point. If chiral symmetry is broken, either dynamically or explicitly, the running coupling is infrared divergent. Based on a truncation for the quark-gluon vertex Dyson-Schwinger equation which respects the analytically determined infrared behavior, numerical results for the coupled system of the quark propagator and vertex Dyson-Schwinger equation are presented. The resulting quark mass function as well as the vertex function show only a very weak dependence on the current quark mass in the deep infrared. From this we infer by an analysis of the quark-quark scattering kernel a linearly rising quark potential with an almost mass independent string tension in the case of broken chiral symmetry. Enforcing chiral symmetry does lead to a Coulomb type potential. Therefore, we conclude that chiral symmetry breaking and confinement are closely related. Furthermore, we discuss aspects of confinement as the absence of long-range van der Waals forces and Casimir scaling. An examination of experimental data for quarkonia provides further evidence for the viability of the presented mechanism for quark confinement in the Landau gauge.
\end{abstract}

(c) 2008 Elsevier Inc. All rights reserved.

\footnotetext{
* Corresponding author.

E-mail address: christian.fischer@physik.tu-darmstadt.de (C.S. Fischer).
} 


\section{Introduction}

Despite the progress seen in the last years the mechanism for confinement of quarks and gluons into colorless hadrons still lacks a detailed understanding. Based on the old idea that infrared divergences prevent the emission of colored states from color-singlet states, see e.g., Refs. [1,2], different functional approaches have been employed to study the infrared behavior of QCD Green functions. It has turned out that Landau gauge is hereby the most suited one. Restricting oneself to the pure gauge sector it is even possible to make definite statements about the infrared behavior of all $n$-point functions $[3,4]$.

An important result of these investigations is the fact that the running coupling as determined from the Yang-Mills vertex functions shows an infrared fixed point [5-8]. This is obtained by balancing infrared suppressed and infrared-divergent proper Green functions: depending on the number of ghost and gluon legs, many of the one-particle irreducible Green's functions are indeed infrared singular, others are infrared vanishing.

Hereby especially the infrared suppression of the Landau gauge gluon propagator immediately raises the question about the cause of the linearly rising quark potential when expressed in terms of Green functions. Additionally, dynamical breaking of chiral symmetry requires an effective strength of the quark interaction which, with an infrared suppressed gluon propagator, necessitates an enhanced quark-gluon vertex [9].

Therefore, the quark-gluon vertex as the central link between the Yang-Mills and the quark sector of QCD is at the focus of many contemporary studies. It is also an important ingredient into the quarkantiquark interaction that is responsible for the formation and properties of bound states. On a perturbative level, the quark-gluon vertex has been studied in detail in arbitrary gauge and dimensions in [10]. In this work, however, we are interested in the non-perturbative properties of this vertex. Lattice studies have granted some first insights into the relative importance of several components of the vertex at intermediate momenta $[11,12]$. In an attempt to understand these lattice studies some of us $[13,14]$ and others [15-17] have presented model studies based on resummations of one-loop results. As we will explain below such a treatment is not sufficient, consequently, a fully self-consistent treatment is required.

An, at least at first sight, surprising result of such a self-consistent treatment is that dynamical chiral symmetry breaking takes place in quite a non-trivial way also in the quark-gluon vertex: this vertex non-perturbatively assumes components of a Dirac scalar type in a similar self-consistent fashion as the quark propagator does. Especially, the assignment of a definite Lorentz structure is then not plausible. In view of the fact that the Lorentz nature of confinement, apparently indicated by phenomenological spin splittings, has worried two generations of QCD practitioners (and is still of interest [18-22]) this result appears, a posteriori, less surprising.

One of our important findings to be reported in this paper is that these parts of the quark-gluon vertex have a considerable strength in the infrared. In particular infrared singularities arise not only in the uniform limit when all external momenta tend to zero, but also when only the gluon momentum vanishes. Interestingly, this soft-gluon singularity is, in contrast to those in the Yang-Mills sector [23], as strong as the uniform divergence. This implies, of course, a complicated structure of the effective low-energy quark-quark interaction with the potential to permanently confine quarks. The remarks made above provide more than ample motivation for a concise study of the Landau gauge quark-gluon vertex. As will become evident below such an investigation reveals many unexpected qualitative features.

This paper is organized as follows: in Section 2 we introduce our notations and discuss the structure of the functional equations for the quark-gluon vertex. In Section 3 we then develop an analytical infrared analysis for the vertex-DSE built upon our previous experience with the Yang-Mills sector of QCD. We discuss self-consistent power law solutions of these equations in the cases of broken and restored chiral symmetry, investigate the corresponding running coupling and determine possible dependencies of the infrared solutions on the quark masses. In Section 4 we then present our numerical results thus confirming our analytical study. The infrared-divergent behavior of the quark-gluon vertex found in our analysis implies a linear rising quark-antiquark potential extracted from the quark four-point function. This is demonstrated in Section 5. In the following Section 6 we deal with theo- 
retical aspects of this confining potential like Casimir scaling and $\mathrm{N}$-ality of the string tension. Phenomenological aspects are discussed in Section 7, before we end with a summary and conclusions. Many of the details of the corresponding calculations are relegated to various appendices.

\section{The quark-gluon vertex Dyson-Schwinger equation}

The fundamental dynamical element in our improved analysis of the quark dynamics in quenched QCD is the quark-gluon vertex. In this section we discuss its basic definition, the functional equations it obeys as well as suitable approximation schemes.

\subsection{Basics}

The dressed quark-gluon vertex

$$
\operatorname{ig} T_{i j}^{a} \Gamma^{\mu}\left(p_{1}^{2}, p_{2}^{2}, p_{1} \cdot p_{2}\right)=
$$

is given by a combination of the 12 independent tensors that can be built from the two independent momenta $p_{1}$ and $p_{2}$ and the Dirac gamma matrices. Since the pioneering paper by Ball and Chiu [24] it is somewhat standard to divide this basis into a part transverse to the gluon momentum, $T_{i=1 \ldots 8}$, and a so called "longitudinal" part $L_{i=1 \ldots 4}$. The latter is not really longitudinal as it contains both longitudinal and transverse parts. The resulting basis is complete but not orthonormal and is explicitly given in Eq. (A1) in the appendix. It contains the tree level tensor $\gamma_{\mu}$ that is a vector in Dirac space as well as scalar parts like $\mathrm{i}\left(p_{1}+p_{2}\right)^{\mu}$ that are not chirally symmetric and are analogous to a scalar mass term in the quark propagator. Introducing a set of scalar functions $\lambda_{i}, \tau_{i}$ of the three scalar variables $p_{1}^{2}, p_{2}^{2}$, $\left(p_{2}-p_{1}\right)^{2}$, the vertex reads

$$
\Gamma^{\mu}=\sum_{i=1}^{4} \lambda_{i} L_{i}^{\mu}+\sum_{i=1}^{8} \tau_{i} T_{i}^{\mu} .
$$

In the course of this paper we will give numerical results for the dressing functions $\lambda_{i}$ and $\tau_{i}$. However, to demonstrate certain properties of the vertex at some places we resort to ansätze for these functions. To avoid confusion in the diagrammatics we represent the quark-gluon vertex by an open circle

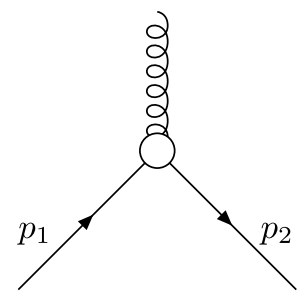

whenever we use an ansatz instead of the fully calculated vertex given in Eq. (1).

In addition we use the following diagrammatic expressions for the full propagators of $\mathrm{QCD}$. The dressed quark propagator is given by 


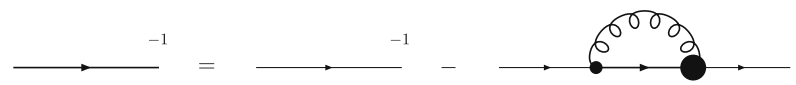

Fig. 1. DSE for the quark propagator including one bare and one proper quark-gluon vertex.

$$
\frac{i}{p}=\left(\mathrm{i} \not p \sigma_{\mathrm{v}}\left(p^{2}\right)+\sigma_{\mathrm{s}}\left(p^{2}\right)\right) \delta_{i j}^{\text {color }}
$$

with the vector part $\sigma_{\mathrm{v}}\left(p^{2}\right)$ and the scalar part $\sigma_{\mathrm{s}}\left(p^{2}\right)$. These functions can be expressed in terms of the quark mass function $M\left(p^{2}\right)$ and the quark wave function $Z_{f}\left(p^{2}\right)$ via

$$
\sigma_{\mathrm{v}}\left(p^{2}\right)=\frac{Z_{f}\left(p^{2}\right)}{p^{2}+M^{2}\left(p^{2}\right)} ; \quad \sigma_{\mathrm{s}}\left(p^{2}\right)=\frac{Z_{f}\left(p^{2}\right) M\left(p^{2}\right)}{p^{2}+M^{2}\left(p^{2}\right)}
$$

that are also expressed in this work in terms of the functions $A\left(p^{2}\right)=1 / Z\left(p^{2}\right)$ and $B\left(p^{2}\right)=Z\left(p^{2}\right) M\left(p^{2}\right)$. The dressed gluon propagator is given by

$$
{\underset{\mu}{a}}_{p}^{b}=P_{\mu v}(p) \frac{Z\left(p^{2}\right)}{p^{2}} \delta_{a b}^{\text {color }}
$$

with the gluon dressing function $Z\left(p^{2}\right)$ and the transverse projector $P_{\mu v}(p)=\left(\delta_{\mu \nu}-\frac{p_{\mu} p_{v}}{p^{2}}\right)$. Finally, the ghost propagator is given by

$$
\frac{a}{\mu}-\frac{b}{\nu}=-\frac{G\left(p^{2}\right)}{p^{2}} \delta_{a b}^{\text {color }} \text {. }
$$

It should be stressed that throughout this manuscript all propagators appearing in loop diagrams are to be taken as dressed, although we do not write the full circles in these cases so as not to clutter the diagrams. We also frequently refer to higher Green's function with more than three external legs. Here we generally denote one-particle irreducible (1PI) and connected functions by filled circles and ellipses, respectively, whereas connected or two-particle irreducible functions (2PI) are denoted by filled rectangles.

\subsection{Functional equations}

The Dyson-Schwinger equations (DSEs) are given as an infinite tower of coupled nonlinear integral equations, see [25-28] for reviews. Therefore, most explicit computations require a specification of an approximation scheme. For such a scheme it is desirable to respect all symmetries of the underlying theory. ${ }^{1}$

The simplest DSE in the quark sector is the one for the quark propagator given in Fig. 1 which involves one bare and one dressed quark-gluon vertex. This equation has been studied in numerous works in the past. Of particular relevance for our work is Ref. [9], where it has been shown that the vertex needs to be enhanced in the non-perturbative momentum region to provide for dynamical chiral symmetry breaking.

The quark-gluon vertex satisfies a full Dyson-Schwinger equation given in Fig. 2 (see [2] and references therein). This equation couples the proper quark-gluon vertex to higher connected four and five-point functions (the quark-gluon and quark-ghost scattering kernel, the two-quark-three-gluon scattering kernel, and the quark-quark scattering kernel). In terms of one-particle irreducible (1PI) Green's functions the DSE, Fig. 2, can be rewritten as given in Fig. 3. These equations can also be directly derived in graphical form as outlined in [29]. In both equations it is the external gluon leg that is connected to a bare vertex in the loop diagrams. Corresponding equations with external quark legs attached to the bare internal vertex exist. One possibility is given in Fig. 4. Certainly, in the full theory

\footnotetext{
${ }^{1}$ Since the Lagrangian density with minimum derivative number and all the symmetries of QCD is QCD itself, any truncation of the equations of motion either (1) breaks at least one symmetry, or (2) is not derivable from a Lagrangian density, or (3) includes higher derivative terms.
} 


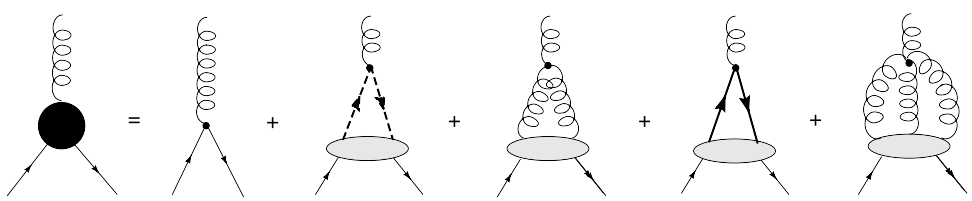

Fig. 2. The DSE for the quark-gluon vertex where the RHS is written in terms of connected Green's functions. All internal propagators are fully dressed, also in all following diagrams. Note symmetry factors $(1 / 2,1 / 6)$ and signs have been omitted.

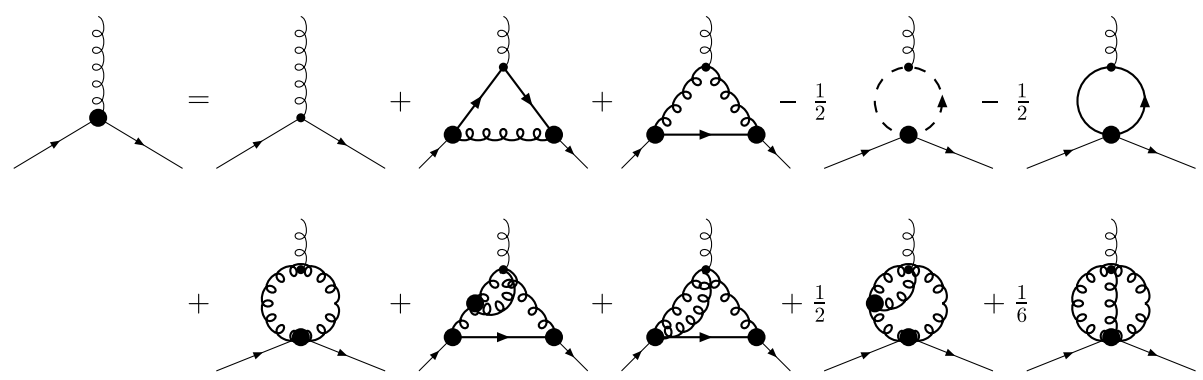

Fig. 3. First version of the DSE for the quark-gluon vertex written in terms of proper Green's functions.

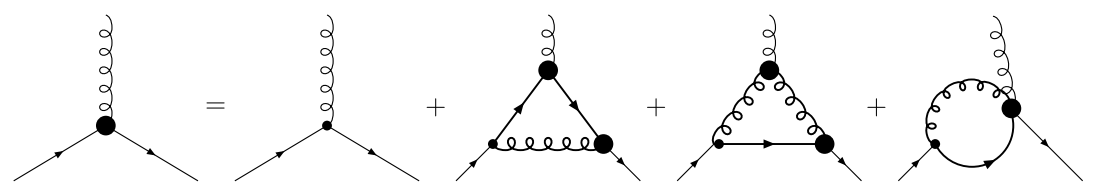

Fig. 4. Second version of the DSE for the quark-gluon vertex written entirely in terms of proper Green's functions.

both equations should give the same quark-gluon vertex. However, in a truncated theory, one of these two equations may be preferred over the other and more straightforward to solve. We deal with this point in Appendix B.

One way to ensure that an approximation scheme respects at least those symmetries that are represented linearly, is to derive it from an action formalism. This holds for schemes based on $n$-particle irreducible ( $n \mathrm{PI}$ ) actions [30]. Such actions depend not only on the averaged field value but also on higher averaged $n$-point correlators thereby generating DSE-like equations via stationarity conditions. They are generalizations of the Cornwall-Jackiw-Tomboulis (CJT) action [31-34] which represents the order $n=2$. Approximations based on the CJT action, which are also referred to as " $\Phi$-derivable", always involve fully dressed propagators whereas approximations based on an $n \mathrm{PI}$-action involve dressed correlation functions with up to $n$ legs.

There are basically two ways to truncate $n$ PI actions based either on loop or large- $N$ expansions. The loop expansion of the 3PI action of QCD has been studied in [30] up to 3-loop order. The result has the form of the standard CJT action which reads formally in Euclidean space

$$
\Gamma_{3 \mathrm{PI}}=S+\frac{1}{2} \mathrm{~S} \operatorname{Tr} \log (\mathcal{D})+\frac{1}{2} \mathrm{~S} \operatorname{Tr}\left(\mathcal{D} \mathcal{D}_{0}^{-1}\right)+\Gamma^{(2)},
$$

where STr is a super trace over the generalized two point function $\mathcal{D}$ for the supermultiplet $\phi$ that includes the fields in the QCD action $\phi=(A, \bar{c}, c, \bar{q}, q)$. The logarithmic term generates fully dressed 1-loop contributions whereas the 2PI-part $\Gamma^{(2)}$ of the action is represented graphically in Figs. 1-4 of Ref. [30]. Self-consistent equations for the Green's functions of the theory are then obtained by variation with respect to the full propagators and vertices. The interesting result is that the truncated selfconsistency equations are nearly identical to the DSE system truncated at the 3-point level when 


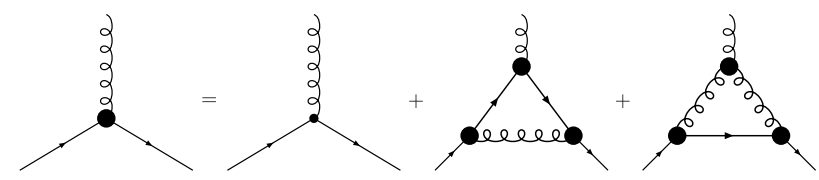

Fig. 5. Equation of motion of the quark-gluon vertex as derived from a 3PI effective action.

neglecting graphs with higher order vertices, but with the decisive difference that all vertices that enter the truncated self-consistency equations are dressed. We will later on confirm that this difference is not crucial when the 3PI equation is compared to the first version, Fig. 3, of the quark-gluon vertex DSE. In Section 3 we will devise a truncation scheme for this DSE which reproduces the infrared behavior of both the full DSE Fig. 2 and the 3PI equation. However, things are slightly more complicated for the second version, Fig. 4, of the quark-gluon vertex DSE. We discuss this point in more detail in Appendix B, where we argue that nevertheless all three equations lead to the same result in the infrared, in the presence of the "soft divergences" studied in Section 3.5 below.

Finally, we would like to mention that another important approach to 1PI Green's functions is given by the functional renormalization group equations (RGEs; for a detailed overview on this technique see $[29,35,36])$. These have been successfully used in the past as an alternative technique to study the infrared behavior of Yang-Mills theory and implications for confinement $[4,8,37,38]$. The functional RGE for the quark-gluon vertex comprises of one-loop diagrams with all vertices dressed. Since this property is also captured by the $n P I$ equations, we will not explicitly discuss these RGEs within this work.

\subsection{Comparison of Abelian and non-Abelian diagram}

The quark-gluon vertex has been investigated in great detail in perturbation theory. Here Davydychev, Osland and Saks gave results for arbitrary gauge and dimension to one loop order and confirmed the validity of the corresponding Slavnov-Taylor identity (STI) [10]. However, as the momentum scale decreases and QCD becomes more strongly coupled, perturbation theory becomes inappropriate. One then has to resort to resummations and finally solve the vertex DSE self-consistently. As a first step it is useful to assess the importance of the different diagrams in the DSEs. This can be done within a semi-perturbative construction based on loop counting in the spirit of a particular type of skeleton expansion [39] in which all propagators are dressed but the internal vertices are still bare. This approximation can also be obtained from a two-loop approximation of the 2PI effective action. It allows us to assess the importance of the diagrams in Fig. 4 on the basis of both, large $N_{c}$ counting and dynamical momentum dependences. In this approximation the DSE in Fig. 4 is given by the diagrams of Fig. 6, which are conveniently labeled the "non-Abelian diagram" and the "Abelian diagram". Whereas the latter diagram has a counterpart in the corresponding DSE for the fermion-photon vertex, the former has not.

These two diagrams and the corresponding semi-perturbative analysis is discussed in detail in Appendix $\mathrm{C}$ and allows to compare the relative strength of the Abelian and non-Abelian diagram numerically. From the color structure of the two diagrams one immediately notices that the Abelian diagram is suppressed by a factor of $N_{c}^{2}$ as compared to the non-Abelian one. Several authors

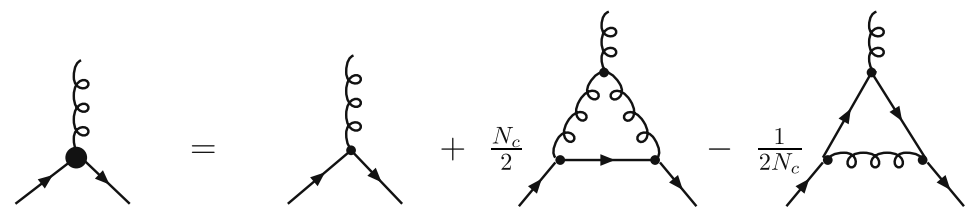

Fig. 6. Semiperturbative approximation to the quark-gluon DSE. The first loop diagram is labeled the "non-Abelian diagram" and the second one "Abelian diagram". All propagators are dressed. 


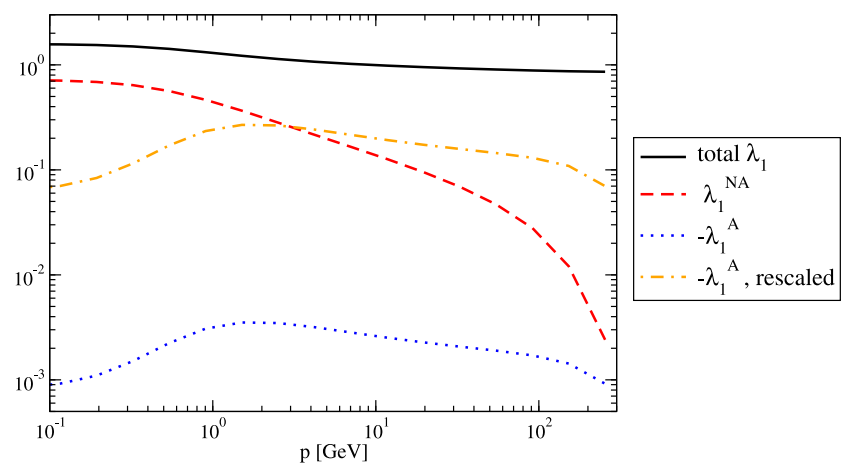

Fig. 7. Dressing factor $\lambda_{1}$ of the $\gamma_{\mu}$ vertex in the semi-perturbative construction with dressed propagators and bare internal vertices. We show the total dressing function $\lambda_{1}=Z_{1 F}+\lambda_{1}^{\mathrm{NA}}+\lambda_{1}^{\mathrm{A}}$ (solid line, renormalized to 1 at $\mu=13 \mathrm{GeV}$ in the soft-gluon kinematic section) as well as the separate contributions $\lambda_{1}^{\mathrm{NA}}$ and $\lambda_{1}^{\mathrm{A}}$ from the non-Abelian and Abelian triangle loops. Finally, we show the Abelian contribution rescaled by an arbitrary factor to compare the momentum dependence.

$[15,16,40,41]$ have recently employed truncations of the quark-gluon vertex based on the $N_{c}$-subleading Abelian one-loop diagram. This is necessary in the context of the Munczek-Nemirovsky model [42] where one substitutes the gluon propagator by a Dirac delta function. Naturally, this operation is not possible in the non-Abelian diagram due to the product of two-delta functions. However, those authors are aware that the Abelian construction is subleading by a factor of $N_{c}^{2}$ and has the wrong sign with respect to the dominant, non-Abelian construction. Therefore, as an ad-hoc fix to alleviate the problem, they have changed the sign of the Abelian diagram and adapted the overall strength to the order of magnitude expected from the non-Abelian graph. The hope is then that the momentum dependence of both one-loop graphs is similar and therefore a calculation of the non-Abelian part can be sidestepped.

We have checked this assumption; see Appendix $C$ for details. Here we only discuss the result for a typical momentum configuration given in Fig. 7. It can be clearly seen that differences remain even after arbitrary rescaling, so this rescaling factor must be dependent on the kinematics and adds to the systematic uncertainties. In general we find from our numerics that the $N_{c}^{2}$ suppression of the Abelian diagram with respect to the non-Abelian one is even amplified by the dynamics.

Another important difference between the Abelian and non-Abelian one-loop diagram (inherited by the semi-perturbative construction) is the quark mass dependence due to the quark propagators. The Abelian diagram contains two quark propagators, the non-Abelian only one. Therefore, upon increasing the quark mass, the diagram's leading pieces fall as $1 / M^{2}$ and $1 / M$, respectively. This behavior cannot be mocked by a constant factor unless this factor depends itself on the mass; but since different Dirac amplitudes depend differently on the quark mass, not all of them can be mocked simultaneously.

Finally, we comment on the potential of this semi-perturbative construction to induce dynamical chiral symmetry breaking in the quark-DSE. To this end we inserted our semi-perturbatively calculated vertex (Abelian plus non-Abelian diagram plus tree-level contribution) into the DSE for the quark propagator, Fig. 1 . As a result we obtained only chirally symmetric solutions with $B\left(p^{2}\right) \equiv 0$. This leads us to the conclusion that semi-perturbative truncations of the vertex-DSE are in general not suited to reproduce the patterns of dynamical chiral symmetry breaking. Consequently we now focus on a more self-consistent approach.

\section{Infrared analysis of the quark sector}

After the general structure of the quark-gluon vertex DSE and its diagrammatic contributions have been studied in the last section via semi-perturbative methods, we will study its interplay in the full set of DSEs of QCD. This is done within a self-consistent, purely analytical, infrared analysis of the coupled system of quark and vertex DSEs, which is later confirmed by our numerical solutions. 


\subsection{Yang-Mills sector}

To begin, we shortly summarize previous results for the infrared behavior of the Green's function in Landau gauge Yang-Mills theory. We discuss the analytical behavior of these functions when all external momenta go to zero. Here, we choose all momenta proportional to each other and require the largest one to be much smaller than the typical scales of the theory, i.e., $p^{2} \ll \Lambda_{\text {QCD }}^{2}$. Here $\Lambda_{\text {QCD }}$ is generated dynamically in the gluon sector of the theory via dimensional transmutation. Then a self-consistent and unique power-law solution of the whole (untruncated) tower of DSEs is given by [3,4]

$$
\Gamma^{n, m}\left(p^{2}\right) \sim\left(p^{2}\right)^{(n-m) \kappa},
$$

where $\Gamma^{n, m}$ stands for the infrared leading dressing function of a Green's functions with $n$ external ghost and antighost legs and $m$ external gluon legs. The exponent $\kappa$ is known to be positive $[7,43]$ but more restrictive limits have been suggested recently [23].

We stress that this infrared (IR) behavior of gauge correlation functions is not influenced by the dynamics of massive quarks as will be confirmed subsequently. We note, however, that due to the possibility of strong divergences in the vertices this does not follow directly from their IR decoupling $[9,44]$. In the heavy quark limit even the value of the scale $\Lambda_{\mathrm{QCD}}$ is determined completely by the gauge dynamics and obeys $\Lambda_{\mathrm{QCD}}^{2} \ll M^{2}$. In contrast, in the chiral limit, where $\Lambda_{\mathrm{QCD}}$ is the unique scale of the system, there could in principle be a different conformal scaling solution in the Yang-Mills sector triggered by quark-loop insertions. This is discussed in more detail elsewhere. Here we concentrate on quenched QCD, where (8) is certainly unaffected by the quark sector.

Examples of the general solution (8) are given by the inverse ghost and gluon dressing functions, $\Gamma^{1,0}\left(p^{2}\right)=G^{-1}\left(p^{2}\right)$ and $\Gamma^{0,2}\left(p^{2}\right)=Z^{-1}\left(p^{2}\right)$, respectively. They are related to the ghost and gluon propagators via Eqs. (5) and (6). The corresponding power laws in the infrared are

$$
G\left(p^{2}\right) \sim\left(p^{2}\right)^{-\kappa}, \quad Z\left(p^{2}\right) \sim\left(p^{2}\right)^{2 \kappa} .
$$

Since $\kappa$ is positive one obtains an infrared diverging ghost propagator, a behavior which is sufficient to ensure a well defined, i.e., unbroken, global color charge [50]. ${ }^{2}$

Note that in Landau gauge an explicit value for $\kappa$ can be derived from the observation that the dressed ghost-gluon vertex remains (almost) bare in the infrared [51]. One then obtains $\kappa=(93-\sqrt{1201}) / 98 \approx 0.595[7,8,52]$, which implies that the gluon propagator vanishes in the infrared. A direct consequence of this behavior are positivity violations in the gluon propagator and therefore the confinement of transverse gluons [5,53]. Another consequence of the solution (8) is the qualitative universality of the running coupling of Yang-Mills theory, see [3] and Section 3.7.

Another important example of the solution (8) is the three-gluon vertex, which later on will serve as input for our analytical and numerical calculations. Restricting ourselves here to dressings of the tree-level structure $\Gamma_{\mu}^{0}\left(p_{1}, p_{2}, p_{3}\right)$ (for a more detailed treatment see [54]), this vertex goes like

$$
\Gamma_{\mu}\left(p_{1}, p_{2}, p_{3}\right) \sim\left(p^{2}\right)^{-3 \kappa} \Gamma_{\mu}^{0}\left(p_{1}, p_{2}, p_{3}\right),
$$

in all kinematical sections where there is only one external scale $p^{2}$, i.e., for $p^{2} \sim p_{1}^{2} \sim p_{2}^{2} \sim p_{3}^{2}$.

As has been noted recently [23,54], there are additional kinematic divergences in the Yang-Mills 3 -point functions that arise when only a single gluon momentum becomes small which we will refer to as the "soft-gluon limit". The IR solution is parameterized by IR exponents $\delta$ for the leading correlation functions in the gauge sector. A lower index denotes the Greens function and the upper indices

\footnotetext{
${ }^{2}$ We emphasize that the Eq. (8) solves the untruncated system of DSEs and the corresponding equations from the functional renormalization group. There is, however, an ongoing discussion, fostered by recent lattice results $[45,46]$ and also continuum investigations $[47,48]$ whether there is an alternative solution to the DSEs with an infrared finite or only weakly diverging ghost propagator and a massive gluon. This discussion involves subtle points of effects of Gribov copies and other artifacts on the lattice as well as in the continuum gauge theory. Pending further clarifications we will ignore this discussion for the moment and proceed by exploring the consequences of Eq. (8) for quenched QCD. We are confident that the results of our investigation, presented below also provides direct support for the validity of the solution (8).
} 
Table 1

The IR exponents for the primitively divergent Green's functions of the unique IR scaling fixed point of Landau gauge Yang-Mills theory

\begin{tabular}{lllllllll}
\hline$\delta_{g h}$ & $\delta_{g l}$ & $\delta_{g g}^{u}$ & $\delta_{3 g}^{u}$ & $\delta_{4 g}^{u}$ & $\delta_{g g}^{g h}$ & $\delta_{g g}^{g l}$ & $\delta_{3 g}^{g l}$ & $\delta_{4 g}^{g l}$ \\
\hline$-\kappa$ & $2 \kappa$ & 0 & $-3 \kappa$ & $-4 \kappa$ & 0 & $1-2 \kappa$ & $1-2 \kappa$ & $1-2 \kappa$ \\
\hline
\end{tabular}

$\delta^{u}, \delta^{g l}$ and $\delta^{g h}$ denote the uniform, soft-gluon and soft-ghost limits. The unique scaling solution is given in Table 1. It involves mild kinematic singularities in the three-gluon vertex $\delta_{3 g}^{g l}$ when only one external gluon becomes soft.

\subsection{Including quarks: basics}

Based on the infrared solution (8) summarized in the last subsection we now extend our analysis to also include the quark sector of QCD. In the following we only consider quenched QCD and only briefly discuss possible effects of unquenching in Section 6.2.

As a starting point we analyze the Dyson-Schwinger equation of the quark propagator, given diagrammatically in Fig. 1. Stripping the color factor, the quark propagator Eq. (3) can alternatively be parameterized as

$$
S(p)=\frac{\mathrm{i} \not p+M\left(p^{2}\right)}{p^{2}+M^{2}\left(p^{2}\right)} Z_{f}\left(p^{2}\right),
$$

with the quark mass function $M\left(p^{2}\right)$ and the wave function $Z_{f}\left(p^{2}\right)$. In this notation the quark-DSE can be written as

$$
\begin{aligned}
& \frac{M\left(p^{2}\right)}{Z_{f}\left(p^{2}\right)}=Z_{2} Z_{m} m+g^{2} C_{F} Z_{1 F} \operatorname{tr} \int \frac{\mathrm{d}^{4} q}{(2 \pi)^{4}} \gamma_{\mu} \frac{M\left(q^{2}\right) Z_{f}\left(q^{2}\right)}{q^{2}+M^{2}\left(q^{2}\right)} \Gamma_{v}(k, q) P_{\mu v} \frac{Z\left(k^{2}\right)}{k^{2}}, \\
& \frac{1}{Z_{f}\left(p^{2}\right)}=Z_{2}+g^{2} C_{F} Z_{1 F} \operatorname{tr} \int \frac{\mathrm{d}^{4} q}{(2 \pi)^{4}} \not p \gamma_{\mu} \frac{\not Z_{f}\left(q^{2}\right)}{q^{2}+M^{2}\left(q^{2}\right)} \Gamma_{v}(k, q) P_{\mu \nu} \frac{Z\left(k^{2}\right)}{p^{2} k^{2}}
\end{aligned}
$$

with $k=p-q$, the Casimir $C_{F}=\left(N_{c}^{2}-1\right) /\left(2 N_{c}\right)$ and the renormalization factors $Z_{2}$ of the quark fields and $Z_{1 F}$ of the quark-gluon vertex. The symbol "tr" denotes the Dirac trace.

Now consider the loop integral on the right hand side of the DSE. When chiral symmetry is broken dynamically we have $M\left(p^{2}\right) \neq 0$ and the denominator of the internal quark propagator cannot be smaller than the generated quark mass $M\left(p^{2}\right)$. As an effect the integral is dominated by momenta larger than the quark mass and the resulting quark mass on the left hand side of the equation freezes out to a constant in the infrared, in self-consistent agreement with $M\left(p^{2}\right) \neq 0$. This infrared screening mechanism of the quark, observed in a broad range of works so far (see [9,55] and references therein), can only be overruled by an extremely singular quark-gluon vertex. We have not found a self-consistent solution for the vertex-DSE that supports such an extreme case and therefore discard this possibility. In general this screening mechanism will also make the quark wave function constant in the infrared, as again observed repeatedly in the literature.

We thus arrive at an IR finite solution of the quark propagator with

$$
M \equiv M\left(p^{2} \rightarrow 0\right) \quad \text { and } \quad Z_{f} \equiv Z_{f}\left(p^{2} \rightarrow 0\right),
$$

where we define the constant and non-vanishing limits by $M, Z_{f}$ and always refer to these limits in the following when no momentum arguments are given. The quark propagator can then be written as

$$
S(p)=\frac{\mathrm{i} \not p+M\left(p^{2}\right)}{p^{2}+M^{2}\left(p^{2}\right)} Z_{f}\left(p^{2}\right) \rightarrow \frac{\mathrm{i} \not p Z_{f}}{M^{2}}+\frac{Z_{f}}{M}
$$


for momenta $p^{2} \ll \Lambda_{\mathrm{QCD}}^{2}$.

In the following we show that a self-consistent solution of the quark-DSE and the DSE for the quark-gluon vertex can be found based on Eq. (15). To this end we now apply the power-counting method of $[3,4]$ to the Dyson-Schwinger equation for the full quark-gluon vertex, given diagrammatically in the first line of Fig. 8. In the second line the higher $n$-point functions of this equation have been expanded to lowest order in a skeleton expansion in terms of full propagators and vertices. The dressed quark-gluon vertex $\Gamma_{\mu}$ can be decomposed in a basis of 12 tensor structures [24] given explicitly in Eq. (A2) in the appendix.

For the internal loops of the skeleton expansion in Fig. 8 there are two possibilities:

(i) they are dominated by loop momenta $q_{\mu}$ of the order of the small external scale $p^{2}$; this then requires infrared singularities in the internal quark-gluon vertices,

(ii) the same screening mechanism applies as in the quark-DSE; for a small external scale $p^{2}$ the loop diagram is then dominated by loop momenta in the mid-momentum region $q^{2} \sim \Lambda_{\mathrm{QCD}}^{2}$ and consequently the vertex freezes out in the infrared.

We emphasize that both possibilities may lead to self-consistent solutions of the quark- and vertex-DSE, although numerically we only found solutions with the property (i). Whether this observation is truncation dependent or not needs to be investigated further in future work. Noting that the second possibility requires no further infrared analysis anyway we proceed to show that indeed self-consistent solutions can be found when (i) is realized.

In this scenario the dressing functions of internal Yang-Mills propagators and vertices can be approximated by the power laws given in Eq. (8). We therefore use Eq. (9) for the scaling of the ghost and gluon propagators. The ghost-gluon vertex scales as a constant in the infrared [51]. The internal quark propagator lines are replaced by (15).

We contracted the right hand side of the DSE with appropriate tensor structures to project out the various components of the vertex, cf. Eq. (A2). Thus, we eventually have to deal with scalar integrals only, which depend on powers of internal and external momenta. The evaluation of such diagrams has been described in [56]. Most important for our purpose is the fact that all powers of internal momenta transform to powers of external momenta after integration. This is also clear from simple dimensional consideration, provided there is only one external scale present. In our case this is ensured by the condition $p^{2} \ll \Lambda_{\mathrm{QCD}}^{2} \leqslant M^{2}$, which leaves only one scale $p^{2}$ in the deep infrared. The following analysis rests on the assumption that there is no second small scale present. In principle this assumption may not be true for heavy quarks, since then

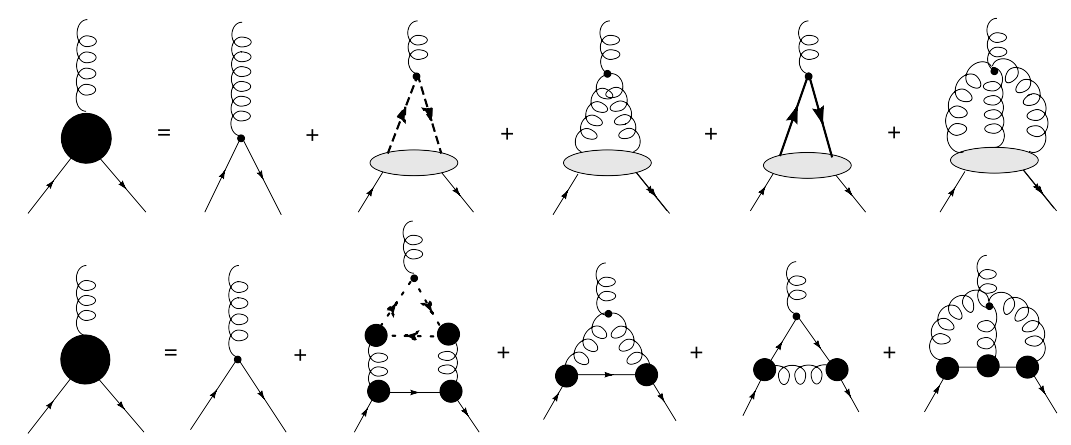

Fig. 8. Dyson-Schwinger equation for the quark-gluon vertex. In the first line we show the full equation [2]. The second line shows important terms of the lowest order of a skeleton expansion, that already reveal the correct power-counting in the infrared. All internal lines in the diagrams represent fully dressed propagators. Symmetry factors and signs have been omitted. The infrared counting analysis can be followed by referring to the second diagram in the right-hand-side of the second line, where one can abstract the ghost loop as if forcing a dressed three-gluon vertex. This is the same counting derived from the last diagram of Fig. 5. 
one has a potential additional small scale $\Lambda_{\mathrm{QCD}}^{2} / M$. We have investigated this possibility in our numerical calculations of the quark-gluon vertex and did not see any influence of such a quantity. The corresponding results of our quark mass study are presented in Section 4.3, where we discuss this issue further.

\subsection{How not to include quarks}

Before we come to our actual solution we would like to point out the complication that the Dirac structure induces. Suppose we only consider a single form factor $\lambda_{1}\left(p^{2}\right)$ with a single IR exponent $\delta_{q g}$ for the quark-gluon vertex corresponding to the tree-level tensor structure $\gamma_{\mu}$. Due to the Dirac-algebra the integral then picks up only contributions from the vector part of the internal quark propagator. The resulting vertex-DSE (IR-leading diagram only), that can be followed from Fig. 8 and its caption, is then given by

$$
\begin{aligned}
\lambda_{1}\left(p_{1}, p_{2}\right)= & \left(\frac{N_{c}}{2} g^{2}\right) \int \frac{\mathrm{d}^{4} q}{(2 \pi)^{4}} \frac{Z\left(k_{1}^{2}\right)}{k_{1}^{2}} \frac{Z\left(k_{2}^{2}\right)}{k_{2}^{2}} \frac{Z_{f}\left(q^{2}\right)}{q^{2}+M^{2}\left(q^{2}\right)} \Gamma^{0,3}\left(k_{1}, k_{2}\right) \\
& \times \lambda_{1}(p, q) \lambda_{1}(q, 2 p)\left(\frac{3 p^{2}}{2}-\frac{5 p^{4}}{4 k_{1}^{2}}-\frac{p^{4}}{4 k_{2}^{2}}+\frac{p^{6}}{4 k_{1}^{2} k_{2}^{2}}\right)
\end{aligned}
$$

with the momenta $k_{1}=p_{1}-q, k_{2}=q-p_{2}$ and the dressing $\Gamma^{0,3}$ of the three gluon vertex. Here we used tree-level tensor structure in the three-gluon vertex without loss of generality. After integration, all powers of internal momenta are converted into powers of external momenta for dimensional reasons (potential conversions into powers of the quark mass are subleading in the infrared and are therefore discarded here; we further justify this treatment a posteriori in our numerical Section 4.3).

Power counting in terms of the external momentum $p^{2}$ then results in

$$
\begin{aligned}
\left(p^{2}\right)^{\delta_{q g}} & \sim p^{4} \times\left[\frac{\left(p^{2}\right)^{2 \kappa}}{p^{2}}\right]^{2} \times \frac{Z_{f}}{M^{2}} \times\left(p^{2}\right)^{-3 \kappa} \times\left[\lambda_{1}\left(p^{2}\right)\right]^{2} \times p^{2} \\
& \sim Z_{f} \frac{\left(p^{2}\right)^{1+\kappa+2 \delta_{g g}}}{M^{2}} .
\end{aligned}
$$

Thus, a self-consistent power-law solution of this equation would entail that

$$
\delta_{q g}=-1-\kappa,
$$

i.e., a singular quark-gluon vertex in the infrared. This solution, however, only serves illustrational purposes, since it does not survive when the full Dirac structure of the vertex included in the other tensor structures is considered. This is shown in the next subsection.

\subsection{Including quarks: the correct way}

To see how the Dirac structure of the vertex can introduce additional complications we note that the tensor structures of the vertex group into two different sets of Dirac-scalar and vector parts, as detailed in Appendix A.1. We have to take into account that within the DSEs the two parts receive qualitatively different contributions from the various loop corrections and introduce distinct IR exponents $\delta_{q g v}$ and $\delta_{q g s}$ for the vector (qgv) and scalar (qgs) Dirac structures. One then arrives at a coupled set of equations with various contributions:

$$
\begin{aligned}
& \left(p^{2}\right)^{\delta_{q g v}} \sim \max \left\{Z_{f} \frac{\left(p^{2}\right)^{1+\kappa+2 \delta_{q g v}}}{M^{2}}, Z_{f} \frac{\left(p^{2}\right)^{1+\kappa+2 \delta_{q g s}}}{M^{2}}, Z_{f} \frac{\left(p^{2}\right)^{1 / 2+\kappa+\delta_{q g v}+\delta_{q g s}}}{M}\right\}, \\
& \left(p^{2}\right)^{\delta_{q g s}} \sim \max \left\{Z_{f} \frac{\left(p^{2}\right)^{1 / 2+\kappa+2 \delta_{q g v}}}{M}, Z_{f} \frac{\left(p^{2}\right)^{1 / 2+\kappa+2 \delta_{q g s}}}{M}, Z_{f} \frac{\left(p^{2}\right)^{1+\kappa+\delta_{g g v}+\delta_{q g s}}}{M^{2}}\right\},
\end{aligned}
$$


where the "max" indicates that the most singular term in the infrared is the leading one on the right hand sides of these equations. This equation can be solved by a range of solutions given by

$$
\delta_{q g v} \in[-1 / 2-\kappa,-\kappa], \quad \delta_{q g s}=-1 / 2-\kappa .
$$

As is demonstrated explicitly in Appendix D, this infrared solution for $\delta_{q g v}$ and $\delta_{q g s}$ does not change when also the tensor components of the quark-gluon vertex are taken into account. The above encountered behavior Eq. (19) is therefore an artifact due to the omission of the scalar Dirac components.

It is furthermore instructive to look closer into the mechanism that generates the solution (21) and recall the properties of the different tensor structures with respect to chiral symmetry. The corresponding transformations act on fermion fields via $\mathrm{e}^{\mathrm{i} \alpha \gamma^{5}} \psi$ with $\alpha$ labeling the transformation. A chirally symmetric quark propagator or quark-gluon vertex would therefore correspond to

$$
\left\{\gamma^{5}, \Gamma^{\mu}\right\}=0 \quad \text { and } \quad\left\{\gamma^{5}, S\right\}=0 .
$$

These relations are satisfied by all tensor structures with an odd number of gamma matrices. Those parts with an even number of gamma matrices can only be present when chiral symmetry is violated. From Eqs. (20) we find that the driving pieces for the self-consistent solution (21) of the vertex DSE are the ones with broken chiral symmetry. If these structures are left out in both the quark propagator and the vertex, one finds a different (infrared weaker) selfconsistent solution, as discussed in Section 3.6 below. $^{3}$ The fact that the presence or absence of chiral symmetry breaking terms generates different solutions of the vertex-DSE seems a remarkable and novel result to us. It underlines that dynamical chiral symmetry breaking is not restricted to the quark propagator alone but happens (consistently) in every Green's function with fermionic content. Before we discuss some aspects of the solution (21), it is important to check that it persists to all orders in the skeleton expansion used in Fig. 8. Higher order terms in this expansion can be generated by inserting diagrammatical pieces like

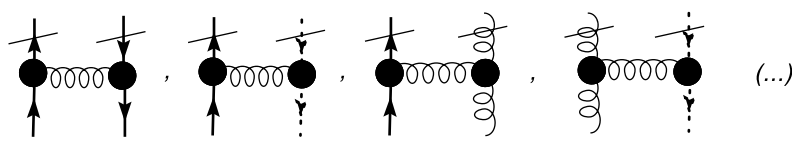

into lower order diagrams (all propagators are fully dressed). In Ref. [3] some of us showed that insertions involving pure Yang-Mills propagators and vertices do not change the overall infrared divergence of a given diagram. This is also true for the three pieces involving quark lines: the first one introduces two quark-gluon vertices, two quark propagators and one gluon propagator plus a new integration into a given diagram. This amounts to an additional power of

$$
Z_{f} \frac{\left(p^{2}\right)^{-1-2 \kappa+2 \kappa-1+2}}{M^{2}}=\frac{Z_{f}}{M^{2}}\left(p^{2}\right)^{0}
$$

in the external momentum $\left(p^{2}\right)$. A similar result is obtained for the other pieces. Thus, diagrammatic insertions of quark lines do not change the infrared behavior of a given diagram but introduce additional powers of $Z_{f} / M^{2}$. This implies that the solution (21) is valid to all orders in the skeleton expansion. It is therefore also an infrared solution of the full vertex-DSE.

Note that similar to the DSEs in the Yang-Mills sector it is the diagram containing the ghost triangle loop that dominates the right hand side of the equation in Fig. 8. Thus, the infrared-divergent

\footnotetext{
${ }^{3}$ Note in this respect that the (inconsistent) behavior Eq. (19) has been obtained from the chiral symmetry breaking part of the propagator while the vertex had only the symmetry preserving term $\gamma_{\mu}$.
} 
behavior of the quark-gluon vertex is an induced effect from singularities in the pure Yang-Mills theory.

Finally, we emphasize that the ghost triangle loop in the infrared leading diagram is also the leading infrared contribution to the fully dressed three-gluon vertex [3]. This offers an interesting opportunity for an approximation which enables our numerical analysis in Section 4. Since we are mainly interested in the infrared behavior of the quark-gluon vertex we may replace the ghost triangle sub-diagram by the dressed three-gluon vertex as shown in Fig. 9. The resulting diagram greatly resembles the "non-Abelian" diagram already discussed above in our semi-perturbative analysis, but with all bare vertices replaced by dressed ones. It is interesting to note that such a completely dressed non-Abelian diagram is also present in the equations of motion for the quark-gluon vertex from the functional renormalization group (FRG) and $n$ PI formulations (with $n>2$ ). This suggests that our infrared solution (21) also solves the corresponding FRG and nPI equations self-consistently. We have checked that this is indeed the case.

\subsection{Soft-gluon singularity in the quark-gluon vertex}

In addition to the main infrared singularity that appears when all scales in a given Green function are sent to zero, there can be kinematical singularities that appear in specific kinematic sections. The present counting rules relied on the implicit assumption that all external momenta $p_{i}$ scale as some common scaling variable $p$. However, in case any of the momenta vanishes identically this is not fulfilled. In the parameterization with a common scaling variable $p$ and dependencies on the other momenta given in terms of dimensionless momentum ratios $p_{i} / p \in(0, \infty)$ or angle cosines between momentum vectors $x_{i} \in(-1,1)$, the IR expression reads

$$
F\left(p_{i} / p, x_{i}\right)\left(p^{2}\right)^{\alpha}, \quad i=1, \ldots, n-1
$$

and these kinematic singularities appear as singularities of the function $F$. We have identified such a kinematic singularity for the quark-gluon vertex in the soft-gluon kinematic section where the gluon momentum $p_{3}=p_{2}-p_{1}=0$, whereas $p_{1}^{2}$ and $p_{2}^{2}$ may be large. In this configuration the singularity is even self-consistently enhanced and thereby is likely to constitute a stable solution of the DSE system. This singularity directly extends the uniform IR singularity discussed above and reported previously in [57] to this larger kinematic section.

The mechanism behind this singularity is based on the strong $\left(p^{2}\right)^{-3 \kappa}$ singularity in the three-gluon vertex when all its momenta vanish simultaneously. To see this consider the infrared leading diagram (9) of the quark-gluon vertex DSE.

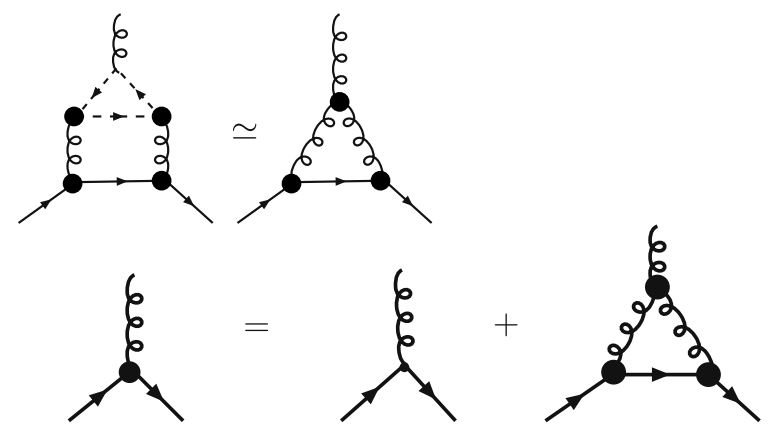

Fig. 9. The infrared leading diagram of the quark-gluon vertex DSE is shown in the first line. In the second line we show an approximation of the full quark-gluon vertex DSE that preserves all infrared features of the equation, while also capturing the leading $N_{c}$-parts of the ultraviolet properties. 


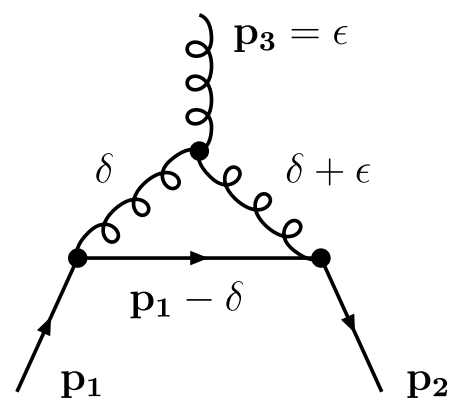

We choose a momentum configuration, where the external momenta $p_{1}^{2}$ and $p_{2}^{2}$ may be large, but the external gluon momentum $p_{3} \equiv \epsilon$ is small. This allows to route the loop momentum $\delta$ to expose the relevant phase-space where both internal gluon momenta, $\delta$ and $\delta+\epsilon$, are small. The incoming quark momentum is fixed at $p_{1}$ and the outgoing is $p_{2}=p_{1}+\epsilon$ : Now, if there are soft-gluon kinematic singularities in the quark-gluon vertex, these also appear in the internal quark-gluon vertices at this particular kinematical configuration and if these singularities are strong enough loop momenta around $\delta$ will dominate the integral (Fig. 10).

Then, leaving out finite factors, self-consistency of the soft-gluon divergence demands that the corresponding exponent $\delta_{q g}^{g l}$ fulfills

$$
\left(\epsilon^{2}\right)^{\delta_{q g}^{g l}} \propto \int \mathrm{d}^{4} \delta\left(\delta^{2}\right)^{2 \kappa-1}\left((\delta+\epsilon)^{2}\right)^{2 \kappa-1}\left(\delta^{2}\right)^{\delta_{q g}^{g l}}\left((\delta+\epsilon)^{2}\right)^{\delta_{q g}^{g l}}\left(\delta^{2}+\epsilon^{2}+(\delta+\epsilon)^{2}\right)^{-3 \kappa} \Gamma_{3 g}^{0},
$$

where $\left(\delta^{2}+\epsilon^{2}+(\delta+\epsilon)^{2}\right)^{-3 \kappa}$ stems from the dressing of the three gluon vertex. Note that the bare three-gluon vertex $\Gamma_{3 g}^{0}$ also contributes a power of momentum $\left(\epsilon^{2}\right)^{1 / 2}$ after integration.

Now, if $\epsilon \rightarrow 0$, the integral is inconsistently divergent in $\delta \rightarrow 0$. Therefore, $\epsilon$ needs to be kept finite, in which case the integral is regulated to the RHS of

$$
\left(\epsilon^{2}\right)^{\delta_{q g}^{g l}} \propto\left(\epsilon^{2}\right)^{\kappa+2 \delta_{q g}^{g l}+1 / 2}
$$

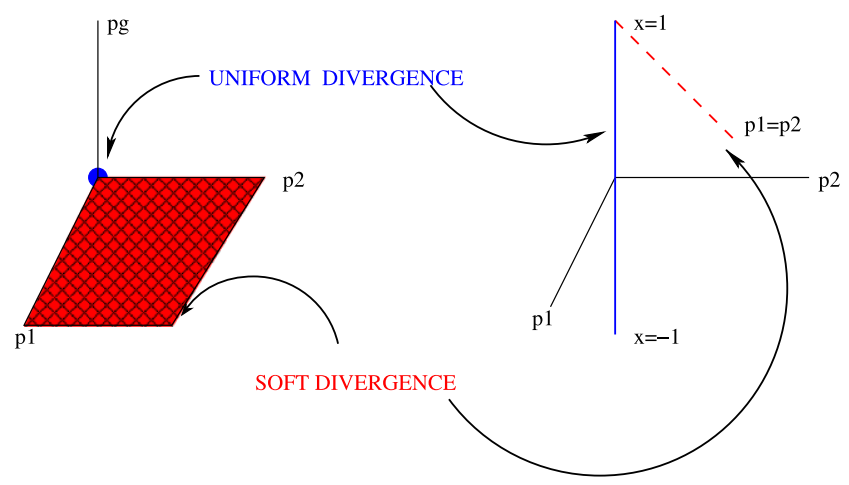

Fig. 10. Support of uniform versus soft IR divergences in the quark-gluon vertex. Left: if the quark-gluon vertex is expressed in terms of the three Lorentz invariants $p_{1}^{2}, p_{2}^{2}, p_{g}^{2}=\left(p_{1}-p_{2}\right)^{2}$, the uniform divergence corresponds to the point where all momenta simultaneously vanish, whereas the soft divergence lies on a plane in this three-dimensional space, since only the gluon momentum is required to vanish. This shows the larger phase-space (and therefore larger contribution to loop integrals) associated with soft divergences. Right: if one uses instead of $p_{g}$ a Euclidean spherical angle between $p_{1}$ and $p_{2}$, whose cosine $x \in(-1,1)$ is on the $O Z$-axis, the uniform divergence corresponds precisely to this segment. However, the soft divergence is now also a segment, at fixed $x=1$, running for $p_{1}=p_{2}$. Thus, a grid optimized to capture only the uniform-divergence in a numerical code will have difficulties to capture the soft-divergence too. 
and therefore it must be that

$$
\delta_{q g}^{g l}=-1 / 2-\kappa
$$

which indeed corresponds to a soft singularity with an equally large exponent in the vertex.

Below, we check explicitly that the counting of soft infrared divergences is not affected by the remaining diagrams in the quark-gluon vertex DSE. In fact the situation is similar to the kinematic section where all external momenta go to zero: in a Dyson-Schwinger formulation the non-Abelian diagram is the infrared leading one with all other diagrams being subleading. In an $n \mathrm{PI}$ formulation or within the FRG framework, where all vertices are dressed in general, we encounter a uniform scaling of the whole equation, i.e., all diagrams have the same infrared divergences.

The importance of the self-consistently enhanced soft-gluon divergence comes from its role for the confinement mechanism of quarks discussed below. A mechanism based purely on the uniform IR divergence as discussed above and proposed in [57] would require the external quarks to have vanishing Euclidean momenta-corresponding to light-like momenta in Minkowski space to trigger the divergence. This is only possible for chiral quarks, whereas any mass would act as a cutoff that shields the divergence. As known from the structure functions measured in deep inelastic scattering experiments [58], the quarks in hadrons have a broad momentum distribution which even vanishes at zero momentum for valence quarks. Similarly, constituent quarks inside charmonia or bottomonia have momenta of the order of or larger than $\Lambda_{Q C D}$ (typically of order $\alpha_{s} M_{Q}$ ). The soft-gluon divergence is realized independent of the quark kinematics and requires only the gluon momentum to become small. Therefore, this type of singularity has the potential to provide a static linear potential between heavy quarks since the momentum of an exchanged gluon is always small as far as the quarks are sufficiently spatially separated. This will be discussed in more detail in Sections 5 and 7.

Let us now analyze the IR power-counting for the full system of DSEs for the quark sector of quenched QCD. In contrast to the propagator which has only one external scale and is parameterized by a single IR exponent $\delta_{q}$, the quark-gluon vertex has two independent external momenta. There are three different limits that can be IR sensitive, namely the uniform limit when all momenta vanish and the soft-gluon and soft-quark limits when only one corresponding momentum vanishes. The corresponding IR exponents are distinguished by upper indices $\delta_{q g}^{u}$, $\delta_{q g}^{g l}$ and $\delta_{q g}^{q}$, respectively. Including all these possibilities in our analysis we can show that the uniform and soft divergences studied before indeed provide a solution of the full non-perturbative system of DSEs (for simplicity we display results only for the one-loop skeleton expansion, but the generalization to an arbitrary order should now be evident, and a skeleton-free proof of the dimensional counting along the lines of [59] possible).

In a case where scales of different order are present the IR scaling law can in principle involve all of them [23]. Since it results from the conformal pure gauge sector, the part in the scaling law that involves powers of the anomalous scaling exponent $\kappa$ always involves the characteristic scale $\Lambda_{\text {QCD }}$. When there are soft $s$ and hard $h$ external momenta, ${ }^{4}$ the scaling law can also involve ratios of them and finally the quark propagators introduce the quark mass $M$. We write all scaling laws in terms of the soft external momentum over $\Lambda_{Q C D}$ and skip any finite ratios of the different hard scales. From the point of view of power-counting the integral can be dominated by modes in the regions around all the external scales. Therefore, it has to be separated into two parts, namely an "infrared" part where the loop momenta are of the order of the soft external momentum $s \ll \Lambda_{\text {QCD }}$ that scales to zero and an 'ultraviolet' part where the loop momenta are either of the order of the quark mass $M>\Lambda_{\text {QCD }}$ or of the order of the hard momentum $h<\Lambda_{Q C D}$ that stays finite in the limit.

The explicit power-counting analysis is presented in Appendix E and yields the following limits:

\footnotetext{
${ }^{4}$ Note one could also speak perhaps more appropriately of "ultrasoft" and "soft" momenta but this might lead to confusion with NRQCD naming.
} 


$$
\begin{aligned}
& \lim _{p \rightarrow 0} S(p) \sim\left(p^{2}\right)^{\delta_{q}} \quad \text { with } \delta_{q}=0, \\
& \lim _{p, q, k \rightarrow 0} \Gamma_{\mu}(p, q, k) \sim\left(k^{2}+p^{2}+q^{2}\right)^{\delta_{q g}^{u}} \Gamma_{\mu}^{0}(p, q, k) \quad \text { with } \delta_{q g}^{u}=\left\{\begin{array}{c}
-\frac{1}{2}-\kappa \\
1-2 \kappa
\end{array},\right. \\
& \lim _{p \rightarrow 0} \Gamma_{\mu}(p, q, k) \sim\left(p^{2}\right)^{\delta_{q g}^{q}} \Gamma_{\mu}^{0}(p, q, k), \\
& \lim _{q \rightarrow 0} \Gamma_{\mu}(p, q, k) \sim\left(q^{2}\right)^{\delta_{q g}^{q}} \Gamma_{\mu}^{0}(p, q, k) \quad \text { with } \delta_{q g}^{q}=0, \\
& \lim _{k \rightarrow 0} \Gamma_{\mu}(p, q, k) \sim\left(k^{2}\right)^{\delta_{q g}^{g l}} \Gamma_{\mu}^{0}(p, q, k) \quad \text { with } \delta_{q g}^{g l}=\left\{\begin{array}{c}
-\frac{1}{2}-\kappa \\
1-2 \kappa
\end{array}\right.
\end{aligned}
$$

where $k$ is the gluon momentum and $p, q$ are the quark momenta. Here $\Gamma_{\mu}$ is the fully dressed quarkgluon vertex and $\Gamma_{\mu}^{0}$ are the undressed tensor structures given in Eq. (A2). Eq. (27) is the central result of our infrared analysis. It shows indeed that the strong dynamics in the quark sector can self-consistently induce both a uniform and a soft-gluon singularity in the quark-gluon vertex, that are of equal strength and which are fully compatible with each other.

\subsection{Restoring chiral symmetry}

The infrared power law solution found in the last subsection is valid in the chirally broken phase of quenched QCD. In the following we will study the chirally symmetric case, i.e., we will omit all those Dirac structures in the propagator and quark-gluon vertex that break chiral symmetry. We still restrict ourselves to the case of quenched QCD, i.e., we do not take into account the backreaction of the chiral quarks onto the Yang-Mills sector. The solution (8) is then still valid. In general, however, unquenching effects are certainly important for chiral quarks and can lead to a different solution in the gauge sector. This possibility will be discussed elsewhere.

With chirally symmetric Dirac structures only, the quark propagator and quark-gluon vertex are given by

$$
S_{\chi}(p)=\frac{\mathrm{i} \not p}{p^{2}} Z_{f}\left(p^{2}\right), \quad \Gamma_{\chi}^{\mu}(p)=\mathrm{ig}\left(\sum_{i=1,2} \lambda_{i}\left(p^{2}\right) L_{i}^{\mu}+\sum_{i=2,3,6,8} \tau_{i}\left(p^{2}\right) T_{i}^{\mu}\right)
$$

in the presence of only one external scale $p^{2}$; the chirally symmetric vertex structures in the general case are given explicitly in the numerical Section 4.5.

For the infrared analysis we project the vertex-DSE on the remaining tensor components. We then arrive at the reduced system for the IR exponents $\delta_{q \chi}$ and $\delta_{q g \chi}$ in the chiral limit

$$
\begin{aligned}
& \left(p^{2}\right)^{-\delta_{q \chi}+1 / 2} \sim \max \left\{\left(p^{2}\right)^{1 / 2},\left(p^{2}\right)^{2 \kappa+\delta_{q g \chi}+\delta_{q \chi}+1 / 2}\right\}, \\
& \left(p^{2}\right)^{\delta_{q g \chi}} \sim \max \left\{\left(p^{2}\right)^{0},\left(p^{2}\right)^{\kappa+2 \delta_{q g \chi}+\delta_{q \chi}}\right\},
\end{aligned}
$$

where $Z_{f}\left(p^{2}\right) \sim\left(p^{2}\right)^{\delta_{q \chi}}$ denotes a potential power law for the quark wave function in the infrared. Assuming for the moment that $Z\left(p^{2}=0\right)=Z_{f}$ stays constant in the infrared, similar to the chirally broken case discussed above, we then find the solution

$$
\delta_{q \chi}=0 ; \quad \delta_{q g \chi}=-\kappa,
$$

i.e., an infrared finite quark wave functions and an infrared-divergent vertex. Note, however, that the vertex is considerably less divergent than in the case of broken chiral symmetry above.

The behavior Eq. (31) is also realized in our numerical solutions, as shown in Section 4.2. We have also investigated the chiral quark-DSE for a range of possible behaviors of the quark-gluon vertex and found that this equation is always controlled by intermediate momenta. In the infrared this naturally leads to a constant $Z_{f}\left(p^{2}=0\right)=Z_{f}$ in agreement with our assumption from above.

In addition to the solution presented above, a pure power-counting analysis cannot exclude the possibility that both the propagator and the vertex scale trivially in the IR limit 


$$
\delta_{q \chi}=0 ; \quad \delta_{q g \chi}=0 .
$$

This possible alternative fixed point is interesting since it would yield an IR vanishing running coupling according to the discussion in the next subsection. A similar behavior has been found previously for the scaling regime of the ungapped magnetic sector at high density [60]. We defer a complete analysis of the fixed point structure for chiral quarks to a future work.

\subsection{The running coupling}

We now wish to investigate the running coupling $\alpha_{q g}$ that can be defined from the quark-gluon vertex. To this end we first recall the corresponding definitions for the couplings from the Yang-Mills sector. Here, renormalization group invariant couplings have been defined from either of the primitively divergent vertices of Yang-Mills-theory, i.e., from the ghost-gluon vertex ( $g g$ ), the three-gluon vertex $(3 g)$ or the four-gluon vertex $(4 g)$ via

$$
\begin{aligned}
& \alpha_{g g}\left(p^{2}\right)=\frac{g^{2}}{4 \pi} G^{2}\left(p^{2}\right) Z\left(p^{2}\right) \stackrel{p^{2} \rightarrow 0}{\sim} \text { const } / N_{c}, \\
& \alpha_{3 g}\left(p^{2}\right)=\frac{g^{2}}{4 \pi}\left[\Gamma^{0,3}\left(p^{2}\right)\right]^{2} Z^{3}\left(p^{2}\right) \stackrel{p^{2} \rightarrow 0}{\sim} \text { const } / N_{c}, \\
& \alpha_{4 g}\left(p^{2}\right)=\frac{g^{2}}{4 \pi} \Gamma^{0,4}\left(p^{2}\right) Z^{2}\left(p^{2}\right) \stackrel{p^{2} \rightarrow 0}{\sim} \text { const } / N_{c} .
\end{aligned}
$$

Using the DSE-solution (8) it is easy to see that all three couplings approach a fixed point in the infrared [3]. This fixed point can be explicitly calculated for the coupling Eq. (33). Employing a bare ghost-gluon vertex one obtains $\alpha_{g g}(0) \approx 8.92 / N_{c}$ [7]. A first step in the evaluation of the coupling of the four gluon vertex has been taken in [61], where the value $\alpha_{4 g}(0) \approx 0.0083 / N_{c}$ has been found.

A non-perturbative and renormalization group invariant definition of the corresponding coupling from the quark-gluon vertex is given by

$$
\alpha_{q g}\left(p^{2}\right)=\frac{g^{2}}{4 \pi} \lambda_{1}^{2}\left(p^{2}\right) Z_{f}^{2}\left(p^{2}\right) Z\left(p^{2}\right) \stackrel{p^{2} \rightarrow 0}{\sim} \frac{1}{p^{2}},
$$

where $\lambda_{1}$ dresses the $\gamma_{\mu}$-part of the vertex. With the exponents $\delta_{q g v}=\delta_{q g s}=-1 / 2-\kappa$, as obtained in our numerical solutions, we find by power-counting that the coupling is proportional to $1 / p^{2}$. Thus, contrary to the couplings from the Yang-Mills vertices, we find this coupling to be singular in the infrared, which corresponds to the old notion of infrared slavery $[1,62,63]$, that has been deemphasized for a decade or two.

If, however, quenched QCD is forced to the chirally symmetric phase as done in the previous subsection, we recover the fixed point behavior also in the coupling from the quark-gluon vertex. Then the weaker infrared singularity (31) of the vertex leads to

$$
\alpha_{q g \chi}\left(p^{2}\right)=\frac{g^{2}}{4 \pi} \lambda_{1}^{2}\left(p^{2}\right) Z_{f}^{2}\left(p^{2}\right) Z\left(p^{2}\right) \stackrel{p^{2} \rightarrow 0}{\sim} \text { const. }
$$

We thus uncovered a close connection between dynamical chiral symmetry breaking and two different possible behaviors of the coupling from the quark-gluon vertex. One obtains infrared slavery when chiral symmetry is broken and a fixed point when chiral symmetry is restored. We will see later on in Section 5 that this connection also extends to the quark-antiquark potential and thus suggests a novel mechanism linking confinement and dynamical chiral symmetry breaking in quenched QCD.

\subsection{Heavy-quark mass dependence of the quark-gluon vertex}

In the previous subsections we discussed in some detail the emergence of self-consistent infrared power laws in the quark-gluon vertex. From our analytical infrared analysis we derived an infrared behavior of $\left(p^{2}\right)^{-1 / 2-\kappa}$ in case of only one external scale $p$ that goes to zero and a soft-gluon infrared divergence of $\left(p_{g}^{2}\right)^{-1 / 2-\kappa}$ in case only the external gluon momentum $p_{g}$ goes to zero. We now investi- 
gate the behavior of the corresponding coefficients and in particular their dependence on the quark mass.

\subsubsection{Naive counting: $M(0) \sim m$}

To this end we start with the vertex constructed in one-loop perturbation theory. Then the internal vertices are not dressed and only feature a color charge $g$ that is universal and independent of the quark mass, with scale-running effects not yet manifest. In first-order perturbation theory, all dependence on the mass comes from the quark propagator, that can be written as

$$
S=\frac{1}{k-M}=\frac{k+M}{k^{2}+M^{2}} \underset{[M \rightarrow \infty]}{\longrightarrow} \frac{1}{M}+\frac{k}{M^{2}} .
$$

Therefore, in the infinite mass limit, the contribution with an even number of Dirac $\gamma$ matrices (the scalar piece) is suppressed by one power of the heavy quark mass, the contribution with an odd number (the vector piece), by two powers.

The behavior of the quark-gluon vertex in perturbation theory, given by Fig. 5 where the dressed vertices are replaced by bare ones, is then easy to obtain. The vector piece is dominant thanks to the bare (mass independent) diagram, and subsequently takes $1 / M^{2}$ corrections from both the Dirac structure $(\gamma / k \gamma) / M^{2}$ of the non-Abelian (second to last) diagram and the Dirac structure $(\gamma M \gamma M \gamma) / M^{4}$ of the Abelian diagram. Likewise the scalar piece has no zero order term, and takes $1 / M$ corrections from the structure $(\gamma M \gamma) / M^{2}$ of the non-Abelian diagram at one-loop. For brevity we will from now on use the symbol $V_{1}$ for the vector part of the vertex (odd number of gamma matrices) and $S_{1}$ for the scalar part (even number of gamma matrices), where the subscript indicates the number of gluon legs. Consequently we will use in the following $V_{0}$ to denote the vector part of the quark propagator and $S_{0}$ for its scalar part, i.e., we have

$$
V_{0} \sim 1 / M^{2}, \quad S_{0} \sim 1 / M
$$

We will read Feynman diagrams following the spin line from left to right. In this notation we can state for the mass dependence at one-loop order

$$
V_{1} \sim O\left(M^{0}\right)+g^{2} O\left(1 / M^{2}\right), \quad S_{1} \sim g^{2} O(1 / M),
$$

where the leading correction is in the scalar piece and arises from the $V_{1} S_{0} V_{1}$-combination in the nonAbelian diagram.

Notice that the Abelian diagram also gives subleading corrections through the $V_{1} V_{0} V_{1} V_{0} V_{1}$-combination and the $V_{1} S_{0} V_{1} V_{0} V_{1}$-combination. In terms of mass dependence these are further suppressed compared to the leading terms (40) and can be ignored. Thus, the conclusion would be that in perturbation theory, heavy quarks increasingly see a Coulombic potential from perturbative one-gluonexchange.

We will now proceed beyond perturbation theory and discuss the leading $N_{c}$, leading skeleton, selfconsistent equation for the vertex as given in Fig. 9. Let us first consider the Dirac amplitudes with an odd number of gamma matrices. The bare term is of order $O\left(M^{0}\right)$ whereas the one-loop skeleton graph on the right gives three types of contributions, namely $V_{1} V_{0} V_{1}, S_{1} S_{0} V_{1}$ and $S_{1} V_{0} S_{1}$. For the even amplitudes, as $S_{1}$, the bare term does not contribute and the one-loop skeleton graph yields structures $S_{1} V_{0} V_{1}, V_{1} S_{0} V_{1}$ and $S_{1} S_{0} S_{1}$.

The entire system is still consistent with the perturbative result, that is, with vertex counting

$$
V_{1} \sim M^{0}, \quad S_{1} \sim M^{-1} .
$$

But now in addition self-consistent solutions arise with

$$
\begin{array}{ll}
V_{1} \sim M^{0}, & S_{1} \sim M^{1} \\
V_{1} \sim M^{1}, & S_{1} \sim M^{1}
\end{array}
$$

for example, the first stemming from the $S_{1}=S_{1} S_{0} S_{1}$ term for the scalar piece and from the $S_{1} S_{0} V_{1}$, $S_{1} V_{0} S_{1}$ terms for the vector piece. Note that the system has no fixed-point solution with $S_{1} \sim M^{n}$ 
with $n>1$ (the $S_{1} S_{0} S_{1}$ term then runs away upon iteration) nor with $V_{1} \sim M^{n}$ with $n>1$. This still leaves us with the situation that, for a self-consistent solution both the scalar and vector pieces grow with the quark mass. As will be seen later on in Section 7 this yields an enhanced string-tension between heavy-quarks that grows quadratically with $M .^{5}$

Let us now back-feed these solutions to the quark-propagator DSE to ascertain whether any change in the perturbative $1 / M$ behavior of the propagator is to be expected. Since the inverse propagator is the amputated Green's function with two quarks and no gluons, by analogy it is obvious that the $1 / M$ counting for higher-point functions (with two quarks, $n$ gluons) is the same. Consider first the vector part of the propagator. We have $V_{0} \geqslant O(1)$ because of the presence of the unsuppressed vector term of the inverse bare propagator $k$ on the right hand side of the DSE. Substituting now $V_{1}=1, M$ in the $\gamma V_{0} V_{1}$ structure of the loop we obtain $M^{-2}$ or $M^{-2} M=M^{-1}$. Thus, the vector part of the propagator remains bare in the heavy-quark limit. Consider now the scalar part of the propagator equation. This gives $S_{0} \geqslant O(M)$ from the bare mass term of the propagator. If we substitute $S_{1}=M$ and $V_{0}=1$ into the self-energy, the relevant structures $\gamma S_{0} V_{1}$ and $\gamma V_{0} S_{1}$ are $O\left(M^{-1}\right)$ and $O\left(M^{0}\right)$, respectively, and therefore subleading compared to the bare mass term. Also, the solution with $V_{1}=1$ yields a power $M^{0}$. We are thus left with the purely perturbative counting in the presence of a heavy quark mass scale, that is,

$$
V_{0} \sim M^{0}, \quad S_{0} \sim M^{1},
$$

consistent with our assumption above.

For the quark-gluon vertex we are thus left with all the three possibilities (41)-(43). It is then a purely dynamical question which of these three possibilities are realized. As a matter of fact there is even a fourth possibility which we discuss now.

\subsubsection{Modified counting: $M(0) \nsim m$}

There is yet another logical possibility that solves the coupled system of propagator and vertex equations, and this is a propagator that is not suppressed by a power of the heavy quark current mass in the infrared, that is, with some degree of saturation in $M\left(p^{2}\right)=B / A$, a mass-independent constant. Substituting this in the vertex equation, it is obvious that self-consistency is reached by a quark-gluon vertex that is also mass-independent, i.e., we have

$$
V_{1} \sim M^{0}, \quad S_{1} \sim M^{0} .
$$

This case has interesting consequences. As will be discussed later on in Section 7, the string tension is then mass-independent. However, in the Schrödinger equation one cannot take the infinite-quark mass limit and simultaneously study the two quarks at infinite distance. The infinitely heavy-quark mass limit only makes sense at intermediate momentum where the propagator is still affected (suppressed) by the heavy quark mass.

As mentioned before, the four cases (41), (42), (43) and (45) are all self-consistent and it is a question of dynamics which one of them is realized. It may even be, that in different regions of the quark mass different scenarios are realized, i.e., one may, for example, encounter the scaling law (45) for physical masses up to the bottom quark mass, whereas the naive perturbative scaling (41) is realized for quark masses as large as the top quark.

In fact, as we will see in Section 4, the scenario (45) is favored by our numerics. The vertex is only mildly dependent on the current quark mass for masses at least up to the bottom quark.

However, this behavior does not persist for larger masses. Our numerical calculations show that for masses in the range of $20-40 \mathrm{GeV}$ the scaling goes over into the perturbative one given by Eq. (41).

\footnotetext{
${ }^{5}$ We presently ignore how the lattice data would rule out such a scenario. It is far from intuitive since lattice practitioners often fix the scale to the string tension, now scaling with the quark mass, and in the heavy mass limit all other splittings they could measure in heavy quarkonium also scale with the mass. Happily experimental data, as seen later, seems to rule this solution out.
} 


\subsection{The Slavnov-Taylor identity}

In this section we check that the infrared power-counting that we have devised satisfies the pertinent Slavnov-Taylor identity (STI) for the quark and gluon vertex. Of course, since our analysis largely hinged on dimensional reasoning one does not expect to find inconsistencies here.

In the following we assume all momenta to be small and proportional to each other, such that our infrared counting rules can be applied.

The Slavnov-Taylor identity for the quark-gluon vertex is explicitly given and derived in [64]. One needs to introduce a non-standard vertex $H^{a}(k, q)$ that corresponds to a ghost-to-quark off-diagonal self-energy. This of course is an auxiliary device that violates all of fermion number, color and momentum conservation. One can think of it as a quark-ghost source. This vertex can be obtained in terms of an equivalent "bare vertex" $g T^{a}$ via an inhomogeneous Bethe-Salpeter equation

$$
H^{a}(k, q)=g T^{a}+B^{a}(k, q)
$$

with the loop integral coupling it to the ghost-quark scattering kernel.

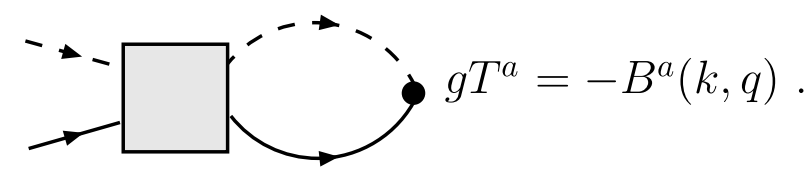

The momenta $k$ and $q$ correspond, respectively, to the incoming ghost and quark legs.

The function $H$ is infrared finite within our power-counting. To see this, expand the quarkghost scattering kernel in terms of a skeleton graph and count the anomalous dimensions of the lowest order using the general counting rule (8) and the infrared leading $1 / M$-terms in the quark-propagator. One arrives then at a total infrared anomalous dimension of $\kappa-1$. This power is precisely canceled by the $-\kappa-1$ from the ghost propagator in the diagram above and the +2 from the loop integration measure. Hence the total exponent is 0 and the function $H$ takes a finite constant value in the infrared.

The Slavnov-Taylor identity for the quark-gluon vertex reads

$$
p_{3}^{\mu} \Gamma^{a}\left(p_{1}, p_{2}\right) \times G^{-1}\left(p_{3}^{2}\right)=H^{a}\left(p_{1}, p_{2}\right) S^{-1}\left(p_{2}\right)-S^{-1}\left(p_{1}\right) H^{a}\left(p_{1}, p_{2}\right)
$$

in terms of the inverse quark propagator $S^{-1}$ and inverse ghost self-energy $G^{-1}$ (note this is written down as $1+b\left(p_{3}^{2}\right)$ in the original reference [64] but there it was assumed to be infrared finite as opposed to infrared suppressed). Since the quark propagator in the chirally symmetric broken phase is a constant and we just argued that $H$ is also an infrared constant, so is the right hand side of the STI.

The left hand side power-law is easy to count: $-1 / 2-\kappa$ from the quark-gluon vertex, $1 / 2$ from the explicit gluon momentum, and $+\kappa$ from the inverse ghost dressing $G^{-1}$ precisely cancel out to yield 0 . Thus, our infrared power-counting is consistent with the requisite gauge invariance. Note also that the argument is independent of whether the quark momentum is small or not, so it applies to either soft or uniform divergence counting, as long as the same counting is used for the quark-gluon vertex and the quark-ghost scattering kernel.

Finally, we discuss the case of the chirally symmetric solution. Here the $\mathrm{H}$-function is still constant since to lowest order in the skeleton expansion there are two powers of $\left(p^{2}\right)^{-1 / 2}$ less in the quarkgluon vertices but also two powers of $1 / p$ more in the quark propagators. The inverse propagator $S^{-1}$ on the right hand side of the STI is now given by i $\not p$, whereas the vertex dressing on the left hand side cancels the contribution of the ghost dressing function. So both the left and the right hand side of the equation are proportional to $p$ and the matching still works.

It is also easy to see how at least the perturbative and modified mass countings for the quark-gluon vertex, for heavy-quark masses, are consistent with the Slavnov-Taylor identity. In the modified counting, neither propagator nor quark-gluon vertex are dependent on the quark mass, therefore 
there is no running in either LHS or RHS of the STI. The perturbative counting can be understood from the Abelian Ward-Takahashi identity in QED

$$
\mathrm{i}\left(k_{\mu}-k_{\mu}^{\prime}\right) \Gamma_{\mu}\left(k, k^{\prime}\right)=S^{-1}(k)-S^{-1}\left(k^{\prime}\right),
$$

where for fixed $k, k^{\prime}$ and $M \rightarrow \infty$ the RHS appears to have a vector part of the fermion propagator $V_{0} \propto M^{0}$, and a scalar part $S_{0} \propto M^{1}$; however, neglecting momentum dependence, the scalar part on the RHS cancels $(M-M=0)$ and since the LHS stays mass-independent, because $\gamma_{\mu}$ is mass-independent, the mass independence of both sides coincides. Coming now to the QCD STI for the qqg vertex in perturbation theory, in this counting the $H^{a}$ kernel becomes a mass-independent constant at large mass (only the $g T^{a}$ bare term survives) and the $M-M$ cancellation happens again on the RHS. Thus, at least the perturbative and modified large-mass countings are seen to be consistent with the STI. For other mass countings the situation is more complicated and we will not discuss them further.

\section{Numerical results for the quark-gluon vertex}

Having discussed the infrared structure of the propagator and vertex DSEs analytically, it is now time to underpin our findings with numerical studies. To this end we solve the coupled system of the DSE for the quark propagator and the one for the quark-gluon vertex in the infrared-preserving approximation discussed around Fig. 9. For the convenience of the reader we display the resulting system again diagrammatically in Fig. 11. In the following we first discuss our numerical techniques and then present our results.

\subsection{Renormalization and numerical methods}

In our numerical calculations we use solutions of the coupled ghost and gluon propagator DSEs as input, cf. Appendix C.3. These have been renormalized at the scale $\mu^{2}=170 \mathrm{GeV}^{2}$, which we also use to renormalize the quark-gluon vertex and the quark propagator. The corresponding renormalization conditions for the quark wave function $Z_{f}\left(p^{2}\right)$ and the vertex $\Gamma_{v}\left(p_{1}^{2}, p_{2}^{2}, p_{3}^{2}\right)$ are

$$
\begin{aligned}
& Z_{f}\left(\mu^{2}\right)=1, \\
& \lambda_{1}\left(\mu^{2}, 2 \mu^{2}, 3 \mu^{3}\right)=1 .
\end{aligned}
$$

Note that only $\lambda_{1}$ appears in this condition, since the accompanying tensor $\gamma_{v}$ is the only divergent part of the quark-gluon vertex; all other dressing functions $\lambda_{2 \ldots 4}$ and $\tau_{1 \ldots 8}$ are finite in the ultraviolet (UV). Note also that we renormalize the vertex at the totally asymmetric momentum slice (cf. Appendix A.2), which is convenient for our numerical setup. The conditions (49) then unambiguously determine the renormalization constants $Z_{2}$ and $Z_{1 F}$ for the quark propagator and the quark-gluon vertex.

The loop integral on the right hand side of the DSE is treated employing standard techniques. We perform all four integrals numerically in hyperspherical coordinates using Gauss-Legendre integration; no angular approximations are done. The radial integral is treated on a logarithmic integration

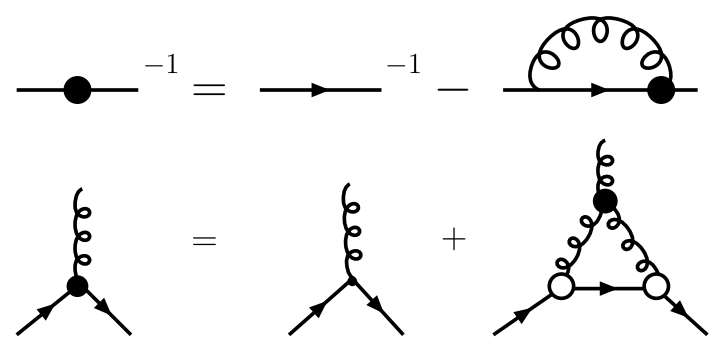

Fig. 11. The DSE for the quark propagator and the quark-gluon vertex as used in our numerical investigation. The open circles in the diagram on the right hand side of the vertex-DSE indicate that we use fit functions for these internal vertices, whereas the quark-gluon vertex in the quark-DSE is the one calculated from the vertex-DSE. 
grid with explicit infrared and ultraviolet momentum cutoffs. We verified that our results are independent of these cutoffs.

The methods to project the right hand side of the vertex equation onto the various tensor structures of the vertex are described in some detail in Appendix F. We evaluate the vertex from the vertex-DSE on a three-dimensional grid, which is adapted to the quark DSE. To this end we use the propagator fits (C9) and (C10) as our initial guess for the quark propagator. Upon backfeeding the vertex into the quark DSE, this equation is iterated until convergence. The resulting quark propagator is then again backfed into the vertex-DSE and so on until complete convergence of the coupled system of equations is achieved.

Due to the considerable numerical complexity we have not yet found a way to completely backfeed the vertex into its own DSE. We therefore employ only parts of its tensor structure for the two internal vertices in the non-Abelian diagram. These are represented by fit functions which are matched against the calculated vertex in the asymmetric kinematical momentum slice. From our infrared analysis in Section 3.4 we saw that in principle it is sufficient to back-feed the most important vector and scalar components into the equation to generate self-consistent infrared physics (at least on a qualitative basis). We therefore employ the two structures

$$
\Gamma_{\mu}\left(p_{1}, p_{2}\right)=\gamma_{\mu} \lambda_{1}\left(p_{1}, p_{2}\right)-\mathrm{i}\left(p_{1}+p_{2}\right)_{\mu} \lambda_{3}\left(p_{1}, p_{2}\right)
$$

for the internal quark-gluon vertices in the non-Abelian diagram. Here we will use the fit forms

$$
\lambda_{1}\left(p_{1}, p_{2}\right)=f^{\mathrm{IR}}(x) g_{1}^{\mathrm{UV}}(x), \quad \lambda_{3}\left(p_{1}, p_{2}\right)=\frac{1}{\sqrt{\left(p_{1}+p_{2}\right)^{2}}} f^{\mathrm{IR}}(x) g_{3}^{\mathrm{UV}}(x)
$$

with $x=p_{1}^{2}+p_{2}^{2}+p_{3}^{2}$. These include the proper asymptotics in the IR and UV

$$
f^{\mathrm{IR}}(x) \equiv\left(\frac{x}{d_{1}+x}\right)^{-\kappa-1 / 2}, g_{1}^{\mathrm{UV}}(x) \equiv\left(\frac{d_{1}}{d_{1}+x}+d_{2} \log \left[\frac{x}{d_{1}}+1\right]\right)^{-9 / 44}, g_{3}^{\mathrm{UV}}(x) \equiv\left(\frac{d_{2}}{d_{2}+x^{n_{1}}}\right)^{n_{2}}\left(\frac{d_{3}}{d_{3}+x}\right)^{2} .
$$

For the three-gluon vertex we use the tree-level tensor structure and represent the corresponding dressing function by

$$
\Gamma^{0,3}\left(p_{1}, p_{2}\right)=\left(\frac{x}{d_{1}+x}\right)^{-3 \kappa}\left(d_{3} \frac{d_{1}}{d_{1}+x}+d_{2} \log \left[\frac{x}{d_{1}}+1\right]\right)^{17 / 44}
$$

which reflects the strong $\left(p^{2}\right)^{-3 \kappa}$ singularity known from Yang-Mills theory, Eqs. (8) and (10) and a logarithmic ultraviolet tail with coefficients from resummed perturbation theory. In all fits the momentum argument is given by the sum of the three squared momenta from the external legs. The fit forms thus represent overall divergences only and do not take care of possible soft gluon divergences. We will modify the fits accordingly when we investigate this issue in Section 4.4 .

In general, backfeeding only parts of the vertex into the vertex equation is somewhat problematic. Since most of the tensor structures of the vertex contribute considerably to the loop integral of the non-Abelian diagram, omitting some of them leads to reduced interaction strength in the integral. As can be seen from our power-counting in Section 3.4 this fact does not affect the qualitative behavior of the resulting vertex dressing functions (i.e., their infrared exponents), but does influence the coefficients of the resulting power laws. As a result one faces a dilemma:

(i) One can insist that the input fit functions match both the output infrared exponents AND their coefficients. We found that this is only possible if our input for the three-gluon vertex is very strong, i.e., much stronger than one would expect from corresponding DSEs for the three-gluon vertex [54]. The missing interaction strength, presumably from the neglected ggg vertex pieces is then simulated by the strong three-gluon interaction. This setup allows one to systematically compare the resulting quark-gluon vertices for different quark masses. However, as we will see, it leads to unnaturally large scales, which cannot be true. 
Table 2

Input parameters for the fit functions (51) and (53) in the chiral limit

\begin{tabular}{lllcll}
\hline & $d_{1}$ & $d_{2}$ & $d_{3}$ & $n_{1}$ & $n_{2}$ \\
\hline$\lambda_{1}$ & 2.00 & 0.5 & - & - & - \\
$\lambda_{3}$ & 4.00 & 0.5 & 0.5 & 1 & -0.5 \\
$\Gamma^{0,3}$ & 1.00 & 0.40 & 10 & & \\
\hline
\end{tabular}

(ii) If we instead insist on correct physical scales, measured, e.g., by the chiral condensate from the quark propagator in the chiral limit, the missing interaction strength in the non-Abelian diagram does not permit a match to the scales from the input fits with the resulting vector and scalar vertex pieces. The missing interaction strength from the neglected vertex pieces in the loop diagram is then taken over by the input dressing functions, which are stronger than the calculated ones.

Naturally this quantitative problem would not exist if we were able to back-feed the complete vertex into the vertex-DSE. Postponing this considerable numerical effort for future work, we will use a combination of the possibilities (i) and (ii) here. We first investigate the chiral limit using the procedure (ii) and thus producing phenomenologically acceptable scales in the resulting vertex and quark propagator. For our quark mass study, however, we have to resort to the procedure (i). Since we are dealing with qualitative statements only, this compromise seems acceptable to us and we believe that the results we obtain are meaningful.

\subsection{The chiral limit}

Let us first have a look at the chiral limit and the corresponding structure of the quark-gluon vertex. The input parameters in our calculation for the fits (51) and (53) are given in Table 2. Here the ultraviolet behavior of the three-gluon vertex is controlled by the parameter $d_{2}$, which is chosen such that the vertex is normalized to the tree-level expression at the renormalization point $\mu^{2}=170 \mathrm{GeV}^{2}$. The corresponding ultraviolet coefficients $d_{2}, d_{3}, n_{1}$ and $n_{2}$ of the input functions $\lambda_{1}$ and $\lambda_{3}$ are chosen such that the calculated vertex dressings are matched by the input. The remaining infrared coefficients $d_{1}$ in $\lambda_{1,3}$ are chosen such that the relative strength of the corresponding calculated dressings is matched. Finally, the parameters $d_{1}$ and $d_{3}$ in $\Gamma^{0,3}$ are chosen such that the chiral condensate of the quark propagator reproduces the phenomenological value.

The resulting input and output vertex dressings for $\lambda_{1}$ and $\lambda_{3}$ are shown in Fig. 12 together with the resulting quark propagator and further vertex dressing functions. In the top right corner we compare the internal representations of the vector $\left(\gamma_{\mu}\right)$ and the scalar $\left(p_{\mu}\right)$ piece of the quark-gluon vertex with the calculated components. The most important observation here is the perfect matching of the generated infrared exponent $\left(p^{2}\right)^{-1 / 2-\kappa}$ of both the $\lambda_{1}$ and $\lambda_{3}$ components with the infrared exponents from the input fits. In fact the very same power law is developed by all 12 tensor structures $\lambda_{1 \ldots 4}$ and $\tau_{1 \ldots .}$. One then expects that backfeeding additional structures besides $\lambda_{1}$ and $\lambda_{3}$ would just reproduce the same infrared exponents as seen from Fig. 12 with backfeeding $\lambda_{1}$ and $\lambda_{3}$ only. We have explicitly checked that this is indeed the case. Thus, the numerical result confirms that all dimensionless vertex dressings share the same infrared divergence. ${ }^{6}$

For the quark mass function $M(p)$ we observe the characteristic rise towards small momenta that is typical for dynamical chiral symmetry breaking. Quite unexpectedly we also observe a turnover at very small momenta, which to our knowledge has not been seen yet in any other truncation scheme. Whether this turnover is a genuine effect independent of the truncation of the vertex-DSE remains to be investigated. Also for the quark wave function $Z_{f}(p)$ we observe a stronger decrease in the infrared than has been observed in previous truncation schemes (see e.g., [9]).

\footnotetext{
${ }^{6}$ In the notation of Appendix D and Ref. [57] this amounts to $\beta_{1}=\beta_{2}=\beta_{3}=\beta_{4}=-1 / 2-\kappa$.
} 

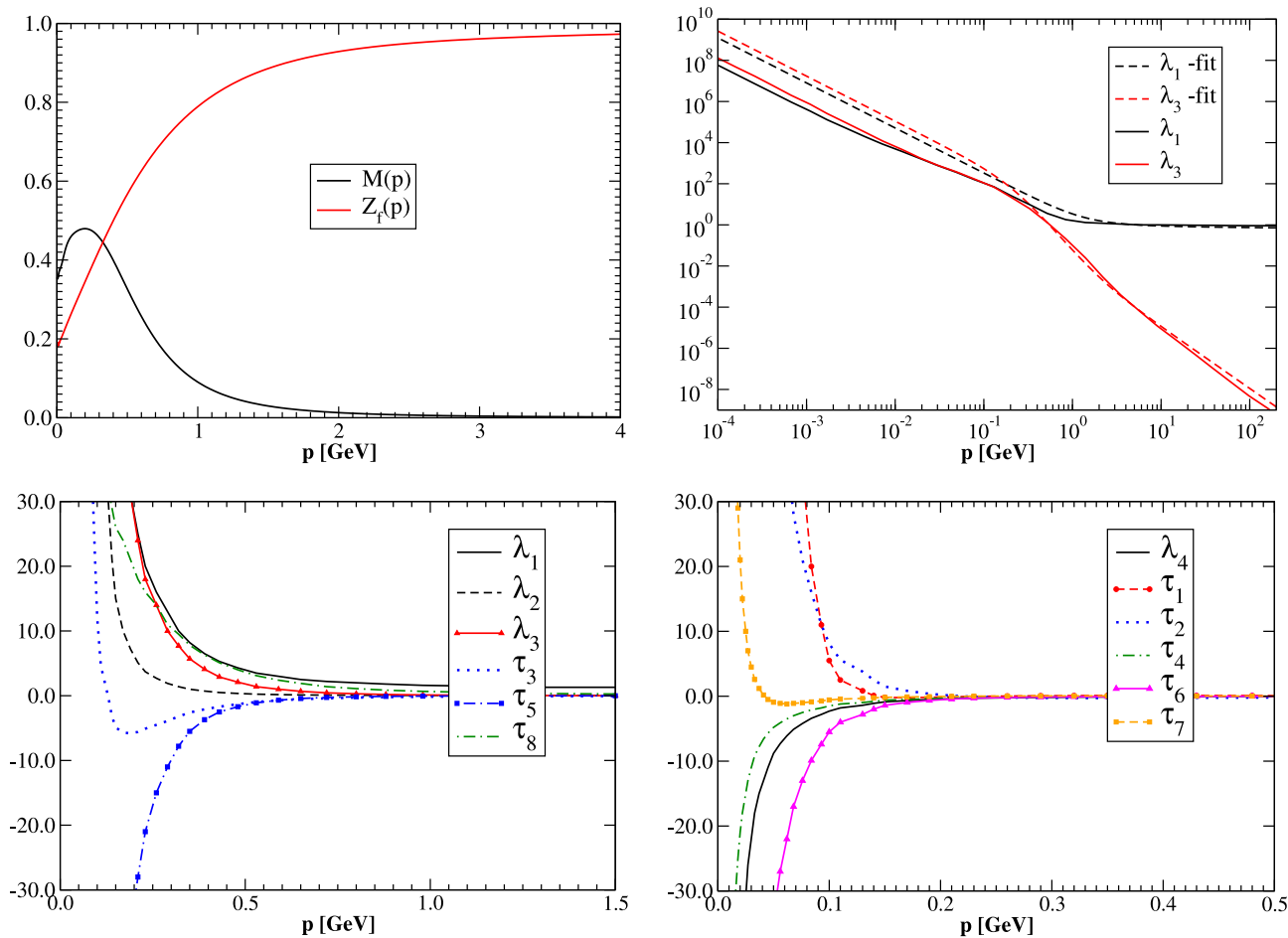

Fig. 12. Top left corner: the chiral quark propagator on a linear momentum scale. Note that the maximum seen in the quark mass function is not a strict prediction of our truncation and may or may not be a truncation artifact. Top right corner: internal representations of the vector and scalar vertex dressings (dashed lines) compared to the corresponding calculated vertex pieces (full lines). Bottom left corner: the six large tensor components of the vertex. Bottom right corner: the six small tensor components of the vertex. Note the different momentum-scale as compared to the plot of the large components. All vertex dressings have been multiplied by appropriate powers of momenta, given in Table 2, such that they correspond to a normalized version of the basis Eq. (2) and can be compared quantitatively. The dressings are plotted versus $p \equiv p_{1}$ and evaluated in the asymmetric momentum slice with $p_{3}^{2}=3 p_{1}^{2}$ and $p_{2}^{2}=2 p_{1}^{2}$.

As concerns the scale we did not match the input and output scales here but pursued scenario (ii) and sought to generate the quark-gluon vertex at a "physical" scale such that the chiral condensate has the correct magnitude. This condensate can be extracted from the quark propagator in the chiral limit according to

$$
-\langle\bar{\Psi} \Psi\rangle(\mu):=Z_{2} Z_{m} N_{c} \operatorname{tr} \int \frac{\mathrm{d}^{4} q}{(2 \pi)^{4}} S_{0}\left(q^{2}\right),
$$

where the trace is over Dirac indices, $S_{0}$ is the quark propagator in the chiral limit and $Z_{m}$ is the quark mass renormalization factor that can also be determined from the quark-DSE. We obtain

$$
\langle\bar{\Psi} \Psi\rangle_{\overline{M S}}^{0, N_{f}=0}(2 \mathrm{GeV})=-(260 \mathrm{MeV})^{3},
$$

where we have used $\Lambda_{\overline{M S}}=225 \mathrm{MeV}$. This value is in agreement with the values reported in the literature.

In the bottom line of Fig. 12 we compare the strengths of the different structures of the vertex. These are multiplied by appropriate factors to make them dimensionless; the factors are given in Table 3. Clearly some structures are more dominant in terms of infrared strength (i.e., coefficients of the universal power law $\left(p^{2}\right)^{-1 / 2-\kappa}$ ), the most important pieces being $\lambda_{1}, \lambda_{3}$ and $\tau_{5} .{ }^{7}$ There is no uniform behavior of the

7 This has already been noted in $[15,16]$. 
Table 3

Dimensionful factors with which the vertex dressings are multiplied to obtain dimensionless quantities

\begin{tabular}{|c|c|c|c|c|c|c|c|c|c|c|c|}
\hline$\lambda_{1}$ & $\lambda_{2}$ & $\lambda_{3}$ & $\lambda_{4}$ & $\tau_{1}$ & $\tau_{2}$ & $\tau_{3}$ & $\tau_{4}$ & $\tau_{5}$ & $\tau_{6}$ & $\tau_{7}$ & $\tau_{8}$ \\
\hline 1 & $4 p_{1}^{2}$ & $2 p_{1}$ & $2 p_{1}$ & $p_{1}^{3}$ & $2 p_{1}^{4}$ & $p_{1}^{2}$ & $4 p_{1}^{3}$ & $p_{1}$ & $2 p_{1}^{2}$ & $2 p_{1}^{3}$ & $2 p_{1}^{2}$ \\
\hline
\end{tabular}

various vertex dressings but some are strictly positive and monotonic, some are strictly negative and some change sign in the infrared. This behavior may or may not be an artifact of the chosen truncation scheme of the quark-gluon vertex DSE and will be investigated further in future work. Of all structures perhaps the most interesting one is the $\lambda_{3}$ piece, since it represents the leading chiral symmetry breaking component of the vertex. Its considerable infrared strength signals a large amount of dynamical chiral symmetry breaking not only in the quark propagator but also in the vertex structures. This is consistent with and in fact corroborates the strategy and results of our infrared analysis in Section 3.4 and Appendix D.

A two-dimensional plot of the dressing function $\lambda_{1}\left(p_{1}, p_{2}\right)$ at fixed angle $\arccos \left(\hat{p}_{1} \cdot \hat{p}_{2}\right)=0.1$ is shown in Fig. 13. One clearly observes that the infrared divergence of $\lambda_{1}$ occurs almost uniformly in the $p_{1}^{2}-p_{2}^{2}$-plane. This is in qualitative agreement with lattice calculations of the vertex [11] at those momenta available on the lattice so far. Unfortunately the volumes of these lattice simulations are by far not large enough to be able to confirm or refute the divergence of the vertex in the deep infrared.

Next we determine the running coupling from the quark-gluon vertex. For the convenience of the reader we repeat its definition here, (cf. Section 3.7):

$$
\alpha_{q g}\left(p^{2}\right)=\frac{g^{2}}{4 \pi} \lambda_{1}^{2}\left(p^{2}\right) Z_{f}^{2}\left(p^{2}\right) Z\left(p^{2}\right) .
$$

Although the right hand side of this definition is renormalization point invariant, it still depends on the renormalization scheme. The scheme we are using, Eq. (49), is adapted to the asymmetric kinematical momentum slice. We call this an "asymmetric MOM-scheme". The resulting running coupling as numerically calculated in this scheme is displayed in Fig. 14. Clearly one sees the usual logarithmic running of the coupling in the ultraviolet, with a value $\alpha_{q g}^{\mathrm{MOM}-\mathrm{asymm}}\left(M_{Z}^{2}\right)=0.118$ at the mass of the Zboson. (Note that the exact agreement of this result with the experimentally determined coupling in the $\overline{M S}$ scheme is incidental.) Towards lower momenta we see a gradual increase of the coupling, where the non-perturbative region $\alpha_{q g}^{\text {MOM-asymm }}>1$ is reached at about $p^{2}=0.75 \mathrm{GeV}^{2}$. The coupling then continually grows, seems to reach a plateau (corresponding to a similar plateau in $\lambda_{1}$ ) and then enters the scaling region in the infrared. In this region, below $p^{2}=(1 \mathrm{MeV})^{2}$ we see a $1 / p^{2}$ singularity

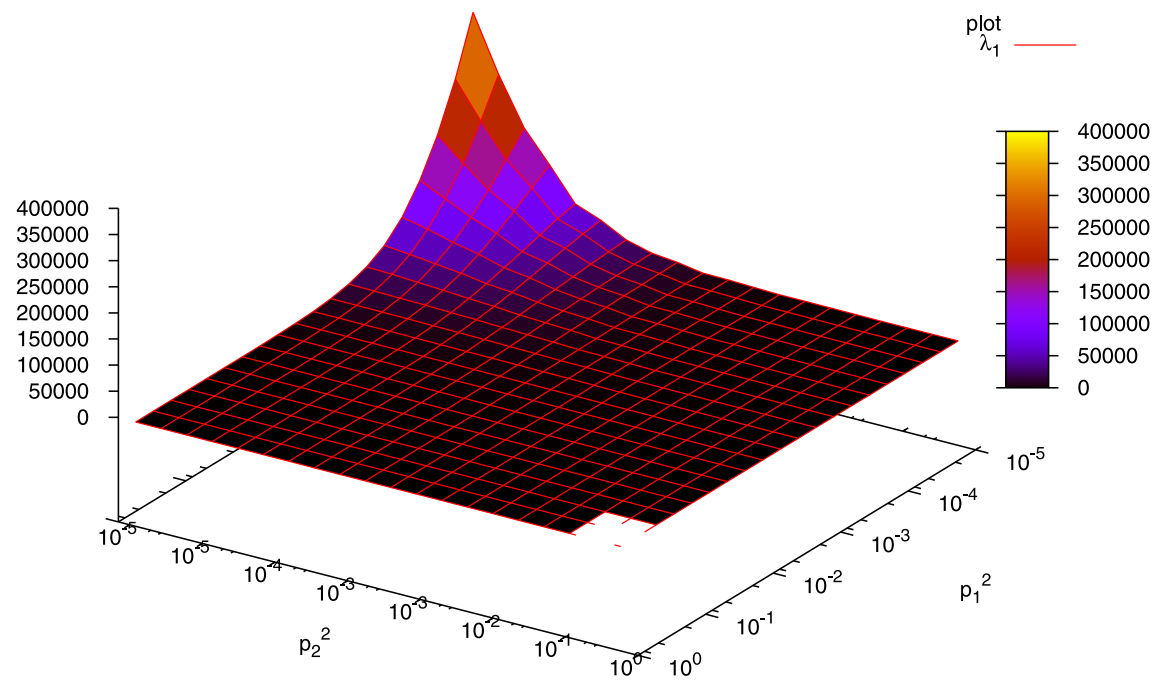

Fig. 13. The vertex dressing function $\lambda_{1}\left(p_{1}, p_{2}\right)$ at fixed angle $\arccos \left(\hat{p}_{1} \cdot \hat{p}_{2}\right)=0.1$. 

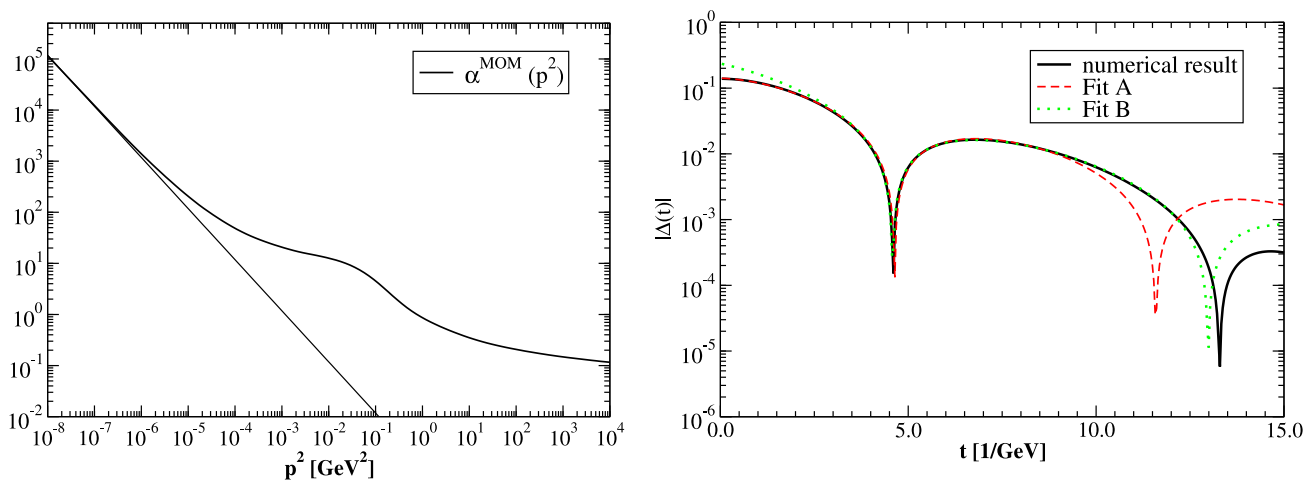

Fig. 14. Left diagram: the running coupling from the quark-gluon vertex in the asymmetric MOM-scheme with $\mu^{2}=p_{1}^{2}=p_{2}^{2} / 2=p_{3}^{2} / 3$. Infrared slavery in the form of a power-law is seen at extremely low-momenta, whereas the coupling is quite flat at intermediate to low-momenta, just as needed for conformal arguments [65]. Right diagram: absolute value of the Schwinger function of the scalar part of the quark propagator in the chiral limit. Clearly visible are positivity violations at a scale of about $5 / \mathrm{GeV} \sim 1 \mathrm{fm}$.

in agreement with our analytical analysis in Section 3.7. Finally, we investigate the possibility of positivity violations in the quark propagator. These are related to the analytic properties of the quark propagator which can in part be read off from the corresponding Schwinger function

$$
\sigma(t)=\int \mathrm{d}^{3} x \int \frac{\mathrm{d}^{4} p}{(2 \pi)^{4}} \exp (\mathrm{i} p \cdot x) \sigma_{\mathrm{S}, \mathrm{V}}\left(p^{2}\right)
$$

where $\sigma_{\mathrm{S}, \mathrm{v}}$ are the scalar and the vector parts, respectively, of the dressed quark propagator. (This method has a long history, see e.g., [53,66-68] and references therein). According to the Osterwalder-Schrader axioms of Euclidean field theory [69], this function has to be positive to allow for asymptotic states in the physical sector of the state space of QCD. Conversely, positivity violations in the Schwinger function show that the corresponding asymptotic states (if present) belong to the unphysical part of the state space. Thus, positivity violations constitute a sufficient condition for confinement. Our results for the Schwinger function of the chiral limit quark propagator are shown in the right diagram of Fig. 14. Shown is the absolute value of the Schwinger function on a logarithmic scale. The thick black curve denotes our numerical result for the Fourier transform (57). Clearly visible is a cusp in the Schwinger function at about $t \approx 5 \mathrm{GeV}^{-1} \approx 1 \mathrm{fm}$. Certainly, this is a typical scale where quark confinement is expected to occur.

A possible analytical structure for such a positivity violating quark propagator has been suggested in [70]. It reads

$$
\sigma(t)=\left|b_{0} \exp \left(-b_{1} t\right) \cos \left(b_{2} t+b_{3}\right)\right|,
$$

which corresponds to a pair of complex conjugate poles of the propagator in the time-like momentum plane. These poles correspond to a "quark mass" given by $m=b_{1} \pm \mathrm{i} b_{2}$. In our case we can fit this form either to the small time behavior of the numerical result or to the region between the first two cusps, as shown in the diagram. The results are then $m=305(5) \pm \mathrm{i} 453(10) \mathrm{MeV}$ for 'Fit $A$ ' and $m=351(5) \pm \mathrm{i} 374(10) \mathrm{MeV}$ for 'Fit B'. Here the errors reflect variations in the bounds of the fitted regions. ${ }^{8}$

\footnotetext{
8 This result demands a comment. In [53] two of us showed that the quark propagator is positive definite if an ansatz for the quark-gluon vertex is used which contains an Abelian part, satisfying the Abelian Ward-Takahashi identity, and an overall nonAbelian dressing function multiplying all tensors uniformly. The resulting DSE for the quark propagator looks similar in structure to the corresponding one for the fermion propagator in QED. Consequently one obtains an analytical structure of a singularity at the fermion mass with a branch cut attached, i.e., exactly the structure expected for a physical electron. It was then left open in Ref. [53] whether the exact quark-gluon interaction of QCD has such a structure, leading to a positive definite quark confined by another mechanism, or whether the quark-gluon interaction is sufficiently different from this ansatz so as to allow for positivity violations. Our results here point in exactly this direction. However, since the relative strength of the vertex components are somewhat truncation dependent further research is necessary to confirm this result.
} 
Table 4

Input parameters for the fit functions (51) and (53) and the current quark mass

\begin{tabular}{|c|c|c|c|c|c|c|c|}
\hline & & $d_{1}$ & $d_{2}$ & $d_{3}$ & $n_{1}$ & $n_{2}$ & $m\left(170 \mathrm{GeV}^{2}\right)$ \\
\hline \multirow[t]{2}{*}{$u$} & $\lambda_{1}$ & 3.51 & 0.5 & - & - & - & 0.003 \\
\hline & $\lambda_{3}$ & 7.20 & 500 & 1.2 & 1 & -1.25 & \\
\hline \multirow[t]{2}{*}{$s$} & $\lambda_{1}$ & 3.22 & 0.5 & - & - & - & 0.050 \\
\hline & $\lambda_{3}$ & 6.65 & 90 & 1.8 & 1 & -1.25 & \\
\hline \multirow[t]{2}{*}{ c } & $\lambda_{1}$ & 1.88 & 0.5 & - & - & - & 1.200 \\
\hline & $\lambda_{3}$ & 3.90 & 18 & 3.0 & 1 & -1.25 & \\
\hline \multirow[t]{2}{*}{$b$} & $\lambda_{1}$ & 2.50 & 0.5 & - & - & - & 4.200 \\
\hline & $\lambda_{3}$ & 5.20 & 0.006 & 0.4 & 4 & -0.3125 & \\
\hline$\Gamma^{0,3}$ & & 3.00 & 0.40 & 1200 & & & \\
\hline
\end{tabular}

The parameters are chosen such that the input fits to $\lambda_{1}$ and $\lambda_{3}$ match the calculated dressing as closely as possible in the asymmetric momentum slice.

\subsection{Mass dependence of the coupled system}

We now investigate the dependence of the quark-gluon vertex on the current quark mass. To compare the results for different quark masses in a meaningful way we match the input and output scales of the vector $\lambda_{1}$ and scalar $\lambda_{3}$ components of the vertex in a given momentum configuration; since the results are qualitatively similar for different kinematical slices, they are most easily presented using the asymmetric momentum slice (cf. Appendix A.2). This choice is not unique, since we saw in a previous subsection that the vertex is not uniform with the angle $\hat{p}_{1} \cdot \hat{p}_{2}$ in the infrared, whereas our input fits are. However, we checked that different choices would not affect the qualitative statements of this subsection, so the asymmetric slice can be chosen without loss of generality.

The input parameters in our calculation for the fits (51) and (53) and the current quark mass are given in Table 4. Again the ultraviolet scale of the three-gluon vertex is matched such that the vertex goes bare at the renormalization point $\mu^{2}=170 \mathrm{GeV}^{2}$. The infrared strength of this vertex is now considerably stronger than in the previous sections: the vertex now effectively takes over the missing strength from the vertex structures which are not backfed into the loop integral. The remaining parameters in the $\lambda_{1,3}$ input fits are then chosen to match their calculated counterparts. Since we are matching in a particular kinematic slice only, it does not make sense to seek for perfect matching here; we therefore concentrated on the infrared power law coefficients and the ultraviolet tails and left some discrepancies at the intermediate momenta. These discrepancies are irrelevant for the points we wish to make here.
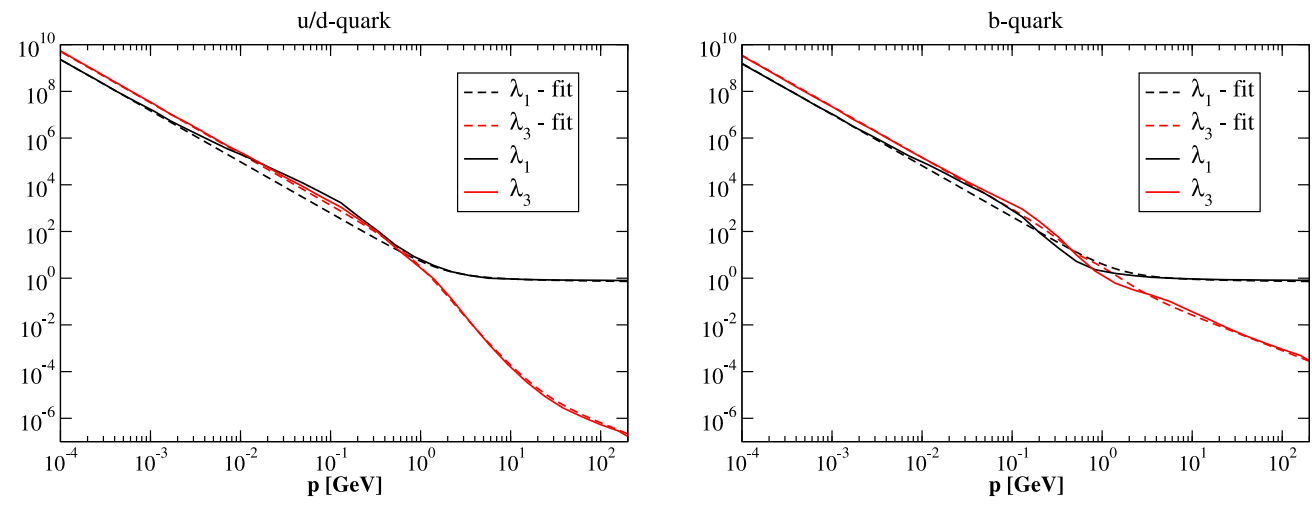

Fig. 15. Internal representations of the vector and scalar vertex dressings (dashed lines) compared to the corresponding calculated vertex pieces (full lines). Shown is the asymmetric momentum slice with $p_{3}^{2}=3 p_{1}^{2}$ and $p_{2}^{2}=2 p_{1}^{2}$. 
The resulting input and output vertex dressings for the two extreme cases ( $\mathrm{u} / \mathrm{d}$ and bottom quark) are shown in Fig. 15.

One observes the following:

- In the infrared the calculated vertex dressings reproduce the power laws from the internal fits regardless of the quark mass. This observation nicely confirms our infrared analysis from Section 3.4 and underlines the presence of universal power laws with mass independent exponents in the infrared.

- We now come back to a point already discussed at the end of Section 3.2: The presence of these mass independent infrared anomalous dimensions also shows that the potentially small scale $\Lambda_{\mathrm{QCD}} / M$ is not small enough (at least for physical quark masses) to disturb the power-counting in the infrared. In fact we do not see any effects from this scale in our numerical results at all. This again justifies a posteriori our infrared analysis in Sections 3.4 and 4.4.

- Comparing the coefficients of these power laws, given in Table 5, we see a much smaller dependence on the quark mass than could have been anticipated from the naive analysis in Section 3.8. Assuming some systematic errors one could interpret the results for both, the vector $\lambda_{1}$ and the scalar $\lambda_{3}$ dressing function as constant if not slightly decreasing with the quark mass. The value of the quark mass function at zero momentum is only slightly increasing with the current quark mass. Clearly, this pattern does not match any of the three possibilities found in our analytical analysis with "naive" counting in Section 3.8.1. Where does the naive counting of quark masses go wrong? The basic assumption of the naive counting is an increase of the infrared mass $M=M\left(p^{2} \ll \Lambda_{\mathrm{QCD}}^{2}\right)$ roughly proportional to the current quark mass. This is, however, not the case in our solutions as can be seen from Fig. 16. This could be in agreement with Section 3.8.2. Whereas the ultraviolet behavior of the quark mass functions indeed is proportional to the current quark mass (as strictly demanded from perturbation theory), the quark mass in the infrared is not.

Table 5

The infrared coefficients for the dressing functions $\lambda_{1,3}$ for different current quark masses $m\left(\mu^{2}\right)$ at the renormalization point $\mu^{2}=170 \mathrm{GeV}$

\begin{tabular}{lllll}
\hline & $u / d$ & $s$ & $c$ & $b$ \\
\hline$m\left(\mu^{2}\right)[\mathrm{MeV}]$ & 3 & 50 & 1200 & 4200 \\
$M(0)[\mathrm{MeV}]$ & 270 & 270 & 320 & 650 \\
$\lambda_{1}\left[\mathrm{GeV}^{1 / 2+\kappa}\right]$ & 3.95 & 3.60 & 2.00 & 2.73 \\
$\lambda_{3}\left[\mathrm{GeV}^{1 / 2+\kappa}\right]$ & 8.70 & 7.97 & 4.44 & 6.08 \\
\hline
\end{tabular}

Also given is the (approximate) value of the mass function at zero momentum.
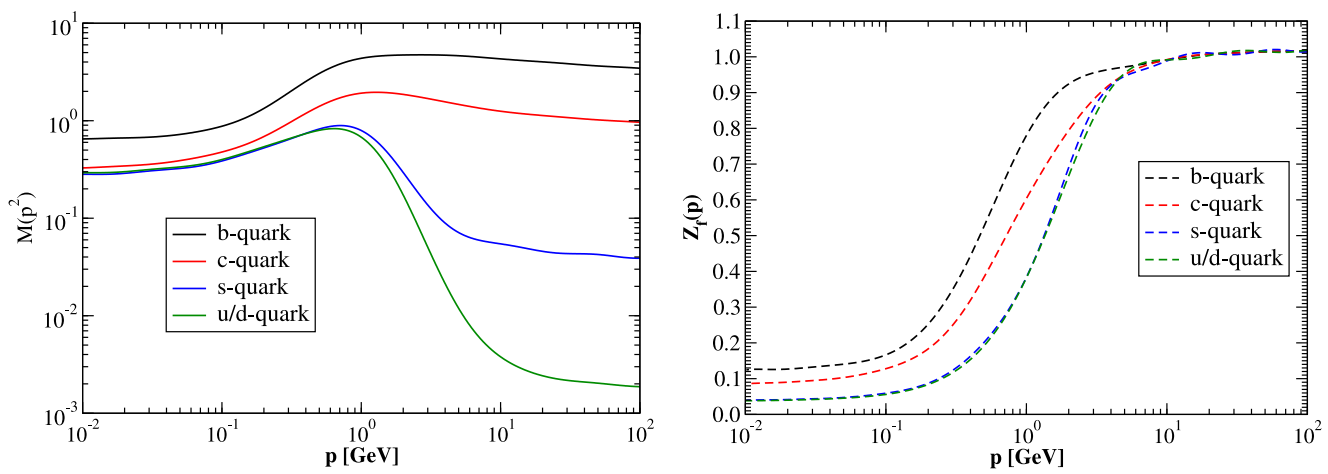

Fig. 16. Color online: The fully converged quark propagator from the coupled system of vertex and propagator DSEs for the different current quark masses (from bottom to top) $m_{u / d}\left(\mu^{2}\right)=3 \mathrm{MeV}, m_{s}\left(\mu^{2}\right)=50 \mathrm{MeV}, m_{c}\left(\mu^{2}\right)=1200 \mathrm{MeV}$ and $m_{b}\left(\mu^{2}\right)=4200 \mathrm{MeV}$ at the renormalization point $\mu^{2}=170 \mathrm{GeV}^{2}$. 
Whereas the current quark mass varies by a factor of $O\left(10^{3}\right)$ from the up- to the bottom-quark mass, the infrared mass $M$ varies only by roughly a factor of two. Together with the variation of $Z_{f}\left(p^{2}\right)$ in the infrared this mass dependence explains the result for the mass (in-)dependence of the quarkgluon vertex observed above.

- We also did calculations for very heavy quark masses in the range of $20-40 \mathrm{GeV}$. In this region, and presumably also for heavier quarks, the naive scaling again sets in, i.e., $M(0) \propto m$ and we see perturbative counting behavior for the vertex. It therefore seems that the region of physical quark masses is clearly distinct.

These two last observations are somewhat unexpected, since they deviate from the usual behavior found in the literature. It remains to be seen whether this effect persists if the quark-gluon vertex is back-coupled self-consistently into its own DSE. Nevertheless it is interesting to speculate of the possible consequences of this result. This will be done in Section 7, where we derive some qualitative aspects of the string tension related to an infrared-divergent quark-gluon vertex.

\subsection{Soft-gluon divergence}

In the last subsection we showed that indeed the quark-gluon vertex as calculated from our vertex-DSE has a strong divergence like $\left(p^{2}\right)^{-1 / 2-\kappa}$ in the infrared once all external scales go to zero, i.e., $p^{2} \sim p_{1}^{2} \sim p_{2}^{2} \sim p_{3}^{3} \rightarrow 0$. However, in our infrared analysis, Section 3.5 we also discovered an additional self-consistent infrared singularity when only the external gluon momentum goes to zero, i.e $p^{2} \sim p_{3}^{2} \rightarrow 0$, but $p_{1}^{2}$ and $p_{2}^{2}$ are kept constant. We now confirm this analytical result also numerically. To this end we change the internal dressing functions (51) to the following forms:

$$
\lambda_{1}\left(p_{1}, p_{2}\right)=f^{\mathrm{IR}}\left(p_{3}^{2}\right) g_{1}^{\mathrm{UV}}(x), \quad \lambda_{3}\left(p_{1}, p_{2}\right)=\frac{1}{\sqrt{\left(p_{1}+p_{2}\right)^{2}}} f^{\mathrm{IR}}\left(p_{3}^{2}\right) g_{3}^{\mathrm{UV}}(x)
$$

which have additional strength in the soft gluon point, i.e., they go like $\left(\left(p_{3}\right)^{2}\right)^{-1 / 2-\kappa}$, where $p_{3}=p_{2}-p_{1}$ is the gluon momentum. Similar as in Eq. (51) we have $x=p_{1}^{2}+p_{2}^{2}+p_{3}^{2}$ for the ultraviolet part of our fits. The parameters are unchanged, i.e., given in Table 2. For the quark propagator we use the chiral result of the last subsection. In Fig. 17 we plot a calculation based on these input forms. Shown is the output dressing function $\lambda_{1}$ in the kinematical section with $p_{1} \cdot p_{3}=0$. We fix $p_{1}=0.24 \mathrm{GeV}$ and vary $p_{2}^{2}$. The soft gluon point $p_{3}^{2}=0$ is then reached when $p_{1}^{2}=p_{2}^{2}$. As can be seen from the plot we clearly encounter a singularity in this limit. From a corresponding log-log plot we

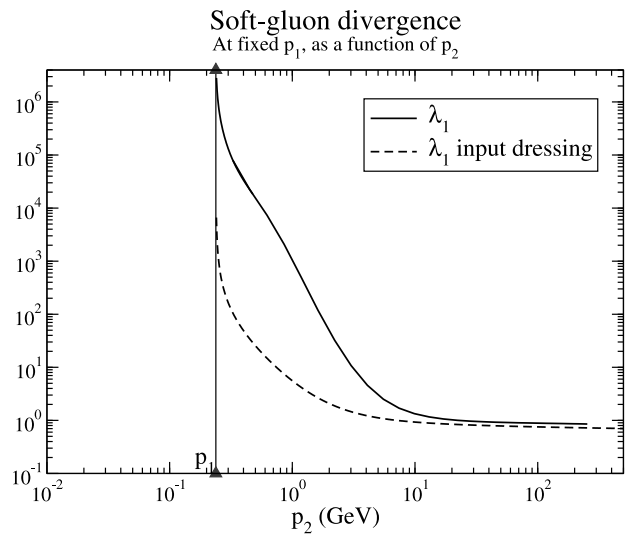

Fig. 17. Input and output of a coupled quark-propagator (totally self-consistent) to quark-gluon vertex (one loop iteration with internal dressings) showing that the soft-singularity is indeed a property of the self-consistent vertex equation with the nonAbelian diagram. 
extracted the exponent of the power law and found $\left(\left(p_{1}-p_{2}\right)^{2}\right)^{-1 / 2-\kappa}$ to good accuracy in agreement with our infrared analysis.

This observation has several important consequences. First, the limit $p_{3}^{2} \rightarrow 0$ is important for the confining potential, as detailed later in Section 7. Second, the soft-gluon endpoint $p_{3}^{2}=0$ is a preferred point chosen in several lattice calculations $[11,12]$. However, we just saw that the vertex is singular at precisely this kinematical section. This then begs the question: how can lattice calculations obtain results at this point.

If it is true that a soft-gluon power law develops, then at fixed gluon momentum $p_{3}=0$ one should obtain an infinite result no matter what quark momentum is chosen. Thus, a computation in the softgluon kinematic section is bound to be infinite. On the lattice, however, this infinity is regulated and one obtains a finite answer for all momenta $p^{2}=p_{1}^{2}=p_{2}^{2}$ at $p_{3}^{2}=0$. This finite answer should then vary with the volume and lattice spacing, provided there is indeed a soft-gluon singularity in the infinitevolume/continuum limit. We therefore propose to study the scaling properties of the vertex in the soft-gluon kinematical section. There is also one further subtle point here: all scaling effects may be wiped out if the vertex is also renormalized in this section (as partly done in [11,12]). Therefore, it is recommended to change the renormalization point to a non-divergent section, as for example our totally asymmetric kinematic section $p_{1}^{2}=1 / 2 p_{2}^{2}=1 / 3 p_{3}^{2}$. Then the graph of $\lambda_{1}$ in the soft-gluon kinematic section is not forced to pass by one, and the sensitivity to varying the grid is exposed.

This exercise is carried out in Fig. 18, where we indeed find a large sensitivity of the results to the grid in the presence of our soft-gluon divergence.

\subsection{Chiral symmetric solution}

Finally, we present numerical solutions for the case of unbroken chiral symmetry. This means we solve the coupled system of quark propagator DSE and vertex-DSE employing chirally symmetric tensor structures only. As detailed in Section 3.6 the quark propagator is then given by

$$
S_{\chi}(p)=\frac{\mathrm{i} \not p}{p^{2}} Z_{f}\left(p^{2}\right)
$$

In general kinematics the corresponding chirally symmetric quark-gluon vertex has the tensor structures $L_{1}, L_{2}, T_{2}, T_{3}, T_{6}$ and $T_{8}$ given in Eq. (A2) with dressing functions $\lambda_{1,2}$ and $\tau_{2,3,6,8}$. All other dressing functions vanish identically. This is reproduced by our numerical solutions.

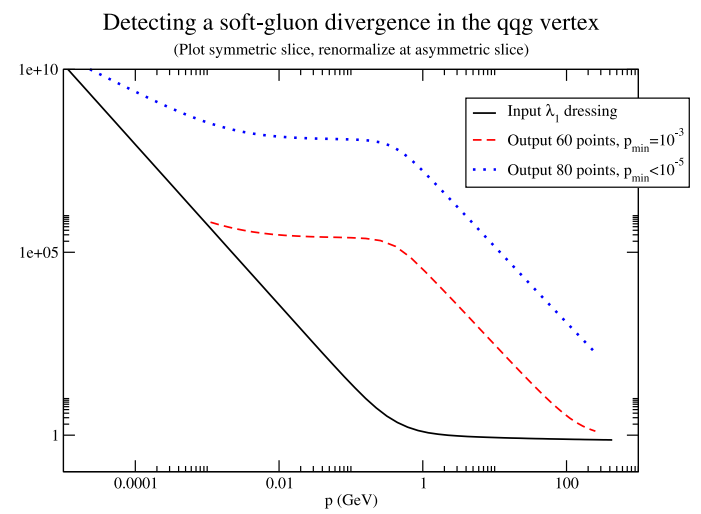

Fig. 18. Black solid line: input $\lambda_{1}$ dressing for the internal vertex with a soft-gluon singularity, plotted here against the momentum of the gluon in a safe kinematic section. The calculation also employs a $\lambda_{3}$ dressing, not shown. Red dashed line: the output $\lambda_{1}$ dressing after one iteration of the non-Abelian loop with the Curtis-Pennington quark propagator as input. The grid has 60 points and is cut-off in the infrared at about $1 \mathrm{MeV}$ (therefore the loop integral too). The plot is in the soft-gluon kinematic section where the analytical result is divergent. Therefore, the curve is an artifact of the grid regularization. Blue dotted line: same but with an 80-point grid and a lower cutoff of $0.01 \mathrm{MeV}$. As seen, the grid sensitivity is large when the renormalization point is not imposed on this kinematic section, forcing the curves to pass by 1 . This spurious effect might be at work in existing lattice calculations. 
Table 6

Input parameters for the fit functions (51) and (53) in the chiral limit

\begin{tabular}{llll}
\hline & $d_{1}$ & $d_{2}$ & $d_{3}$ \\
\hline$\lambda_{1}$ & 0.10 & 0.20 & 0.02 \\
$\Gamma^{0,3}$ & 1.00 & 0.40 & 1.00 \\
\hline
\end{tabular}
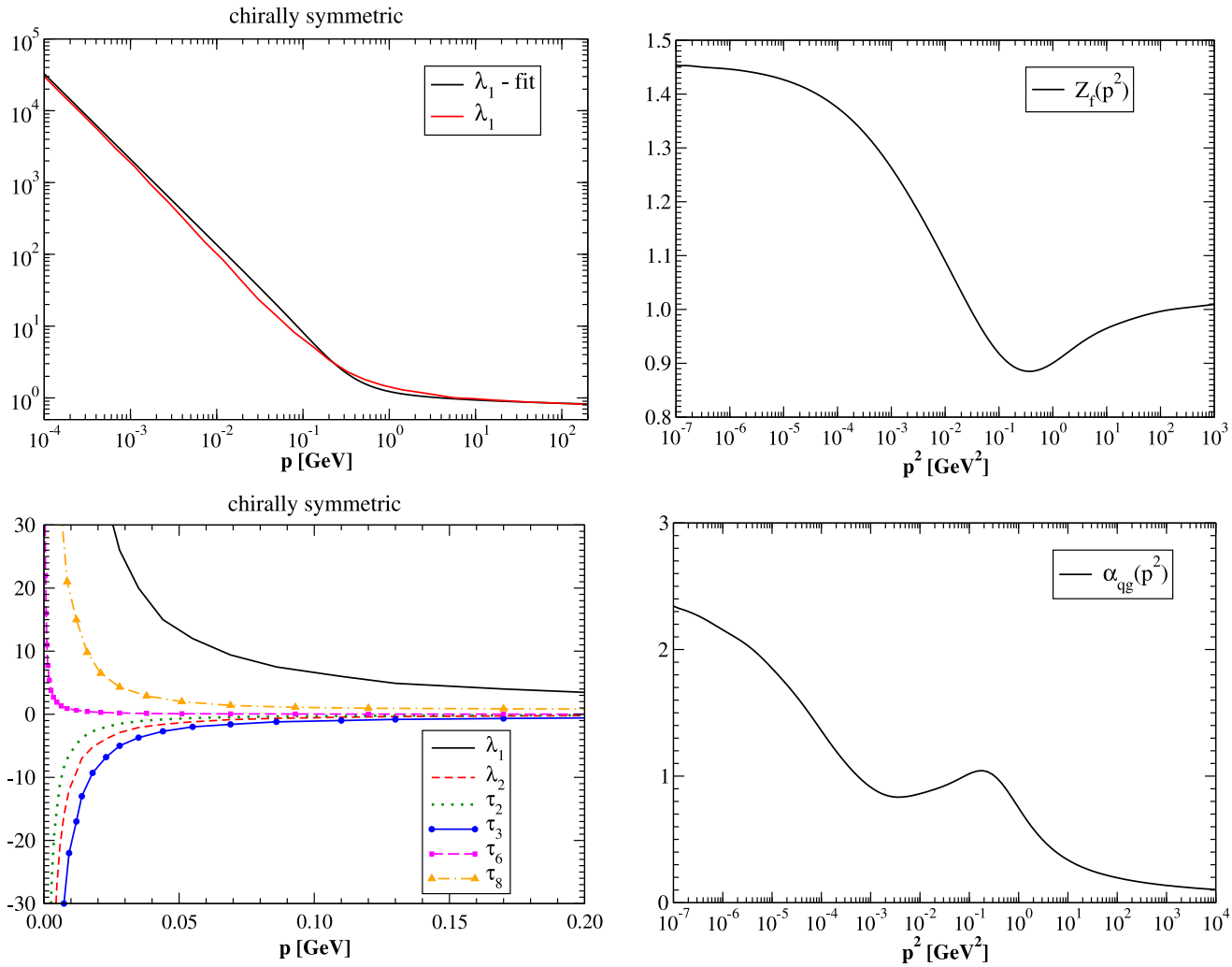

Fig. 19. All diagrams show results for the quark-gluon vertex, the quark propagator and the running coupling in the case where chiral symmetry is forced to be unbroken. Top left corner: internal and external (calculated) dressing function $\lambda_{1}$ in the asymmetric momentum slice with $p_{3}^{2}=3 p_{1}^{2}$ and $p_{2}^{2}=2 p_{1}^{2}$. Top right corner: the quark propagator function $Z_{f}\left(p^{2}\right)$. Bottom left corner: the six chirally symmetric tensor components of the quark-gluon vertex. Bottom right corner: the running coupling for the chirally symmetric case. Note that within numerical errors the coupling approaches a fixed point in the infrared in agreement with our analytical analysis. The bump at mid-momentum is model dependent and potentially an artifact due to scale mismatch between the gluon propagator and the vertex functions.

Since $\lambda_{3}$ is among the vanishing dressing functions we only work with $\lambda_{1}$ in the internal quarkgluon vertices. The corresponding fit function is given by

$$
\lambda_{1}\left(p_{1}, p_{2}\right)=\left(\frac{x}{d_{1}+x}\right)^{-\kappa}\left(d_{3} \frac{d_{1}}{d_{1}+x}+d_{2} \log \left[\frac{x}{d_{1}}+1\right]\right)^{-9 / 44} .
$$

Note that the infrared exponent is changed from $-1 / 2-\kappa$, as in Eq. (51), to $-\kappa$ and we have introduced an additional parameter $d_{3}$. The input for the three-gluon vertex is unchanged compared to (53). Again we matched the input and output values for $\lambda_{1}$ in the asymmetric momentum slice $p^{2}=p_{1}^{2}=p_{2}^{2} / 2=p_{3}^{2} / 3$. The resulting values for the parameters are given in Table 6 . Here the parameters for the three-gluon vertex allowed for the'natural' values $d_{1}=d_{3}=1$. These set the scale of the results. Certainly this scale is arbitrary, since we have no physical situation to compare with. 
Our results for the vertex and the quark propagator are displayed in Fig. 19. In the top left corner we show the matching between the internal fit form for $\lambda_{1}$ and our calculated result for the asymmetric momentum slice. The overall matching is very good, the slight deviations in the mid-momentum regime do not affect the point we would like to make: in the infrared there is a clear matching between the internal and external power laws once again justifying our analytical study from Section 3.6. The vertex indeed diverges in this case with $\left(p^{2}\right)^{-\kappa}$. The resulting quark dressing function $Z_{f}\left(p^{2}\right)$, displayed in the top right corner, shows a somewhat unexpected behavior. It starts with the expected perturbative running in the ultraviolet momentum region. Then it bends down towards smaller momenta in the mid-momentum region similar to the chirally broken case. It then, however, starts rising again and settles for a finite infrared value larger than one. Taken at face value such a behavior forbids a positive definite spectral function [71], i.e., such a quark propagator does not describe a physical quark. Since this behavior may or may not be an artifact of our truncation it creates no problem per se, since the situation we are investigating in this subsection has no counterpart in the real world. For our purposes it is sufficient to note that the quark dressing function $Z_{f}\left(p^{2}\right)$ indeed goes to a constant in the infrared, thus confirming our central assumption of the infrared analysis in Section 3.6.

In the bottom left corner of Fig. 19 we show the dressing of the quark-gluon vertex as a function of the momentum. Similar to the chirally broken case we find $\lambda_{1}, \tau_{3}$ and $\tau_{8}$ among the large vertex components, whereas $\lambda_{2}, \tau_{2}$ and $\tau_{6}$ are somewhat smaller. The running coupling in the chirally symmetric case is shown in the bottom right diagram of Fig. 19. In agreement with our infrared analysis we find something like a fixed point in the infrared. The scaling behavior is not as convincing as in the chirally broken case. To a large extent this may be to numerical uncertainties, which are much larger than in the chirally broken setup. We are working to improve this situation in the future. Note that the precise value of this fixed point depends on the input strength of the three-gluon vertex and is therefore not a prediction of our calculation. It is the mere existence of this fixed point that will be important for our analysis of the quark-antiquark potential later on.

\section{The quark-quark scattering kernel}

In the previous two sections we found an infrared-divergent, self-consistent solution for the quarkgluon vertex using both analytical and numerical methods. We now wish to investigate the consequences of such a behavior for the quark-quark scattering kernel, i.e., the Green's function that should contain the confining quark potential in the heavy quark limit of quenched QCD. We will first briefly discuss the relevance of chiral Ward identities, then discuss the properties of four-quark Green's function in a skeleton expansion (cf. Ref. [57]) and finally show that the results derived from this expansion can also be directly justified from the Dyson-Schwinger equation of the four-quark function.

\subsection{Quark-quark scattering kernel and chiral Ward identities}

In the heavy quark limit chiral symmetry breaking is dominated by the effects of the large bare quark mass. Thus, chiral Ward identities, associated with effects from the dynamical breaking of chiral symmetry, play only a minor role. Certainly, the opposite is true when quarks with small masses are considered. Then, the axial vector Ward-Takahashi identity enforces an intimate relation between the scattering kernel and the gap equation.

A systematic prescription to construct a scattering kernel that is consistent with a given quarkgluon interaction has been given by Munczek [72]. Beyond-rainbow-ladder approaches have been demonstrated, for example in [73], with semiperturbative-type vertex constructions. The basic idea is to take functional derivatives of the quark self-energy with respect to the quark propagator. In Appendix $G$ we detail a truncation scheme of the coupled system of Dyson-Schwinger equations for the quark-gluon vertex, the quark-gluon scattering kernel and the quark propagator DSE that is amenable to Munczek's prescription. We then give a formal expression for a resulting Bethe-Salpeter kernel that is both chirally symmetric and confining. The construction is formally straightforward but leads to a complicated system of equations whose explicit treatment is far beyond the scope of this work. Instead we proceed discussing the heavy quark limit of the scattering kernel, where we expect a skeleton expansion to deliver meaningful results. 


\subsection{Quark-quark scattering kernel in a skeleton expansion}

We investigate the infrared behavior of the full quark-quark four-point function in the heavy quark limit and in the presence of only one dynamical external scale (the distance between a quark and an antiquark). This function together with the first two terms of a skeleton expansion are given in Fig. 20. As in Section 4.4 we examine the infrared behavior of these graphs and perform a scaling analysis in terms of infrared anomalous dimensions.

The first term of Fig. 20 is non-perturbative one-gluon exchange, i.e., one-gluon exchange with a dressed propagator and two dressed quark-gluon vertices. Since we are working in the heavy quark limit with only one small external scale, i.e., the gluon momentum, we are probing the soft singularity of the quark-gluon vertex $\left(k^{2}\right)^{-1 / 2-\kappa}$. We have seen in Section 3.5 that this singularity appears in all tensor structures of the vertex. Thus, we do not need to distinguish between vector and scalar components here.

Together with the gluon propagator scaling $\left(k^{2}\right)^{2 \kappa-1}$ we thus arrive at a total scaling

$$
\text { term } 1 \sim\left(k^{2}\right)^{-2}
$$

of the first term in the skeleton expansion of the four-quark kernel. Now observe how adding the second rung modifies the first term. For the quark propagator we have $S=\frac{i \not p z_{f}}{M^{2}}+\frac{z_{f}}{M} \rightarrow \frac{z_{f}}{M}$ in the infrared which leads to

$$
\text { term } 2 \sim \text { term } 1 \times\left(Z_{f}^{2} \frac{\left(k^{2}\right)^{2+2 \kappa-1+2(-1 / 2-\kappa)}}{M^{2}}+\cdots\right) \sim \frac{Z_{f}^{2}}{M^{2}}\left(k^{2}\right)^{-2}
$$

for the second term. Here the dots represent subleading contributions. The first factor of $\left(k^{2}\right)^{2}$ comes from the four-momentum integral, then we have $\left(k^{2}\right)^{2 k-1}$ from the extra dressed gluon propagator and two scaling factors $\left(k^{2}\right)^{-1 / 2-\kappa}$ from the quark-gluon vertices. We thus obtain the same scaling behavior of the second term than for the first term. With the methods of Section 3 it can be shown that this behavior persists for all other terms of the skeleton expansion.

As a result we obtain for the four-quark kernel the power law $H\left(k^{2}\right) \sim\left(k^{2}\right)^{-2}$. This is in perfect agreement with the $1 / k^{4}$ behavior in the old infrared-slavery scenario. The crucial difference, however, is that in the former scenario the vertices have been assumed constant in the infrared, whereas the gluon was believed to be singular as $1 / k^{4}$. Here we have an infrared vanishing gluon compensated for by the strength of two infrared-divergent quark-gluon vertices.

Then, the well-known relation

$$
V(\mathbf{r})=\frac{1}{(2 \pi)^{3}} \int \mathrm{d}^{3} k H\left(k^{0}=0, \mathbf{k}\right) \mathrm{e}^{\mathrm{i} \mathbf{k r}}=\frac{1}{(2 \pi)^{3}} \int \mathrm{d}^{3} k \frac{\mathrm{e}^{\mathrm{i} \mathbf{k r}}}{\mathbf{k}^{4}} \sim|\mathbf{r}|
$$

between the static four-quark function $H\left(k^{0}=0, \mathbf{k}\right)$ and the quark potential $V(\mathbf{r})$ therefore gives a linearly rising potential by naive dimensional arguments. We therefore find confinement in the chirally broken phase of quenched QCD. A more refined treatment, as described in [74], leads to the same result. By construction already the first term in the skeleton expansion, i.e., non-perturbative one-gluon exchange displayed in Fig. 20, generates this result. Since the following terms in the expansion are equally enhanced in the infrared, the string tension will be built up by summing over an infinite number of diagrams. We will come back to this point below.

It is instructive to also have a look at the chirally symmetric case. The corresponding infrared solutions for the quark propagator and quark-gluon vertex have been derived in Section 3.6 and confirmed numerically in Section 4.5. They correspond to a vertex scaling of $\left(k^{2}\right)^{-\kappa}$ and a gluon propagator which

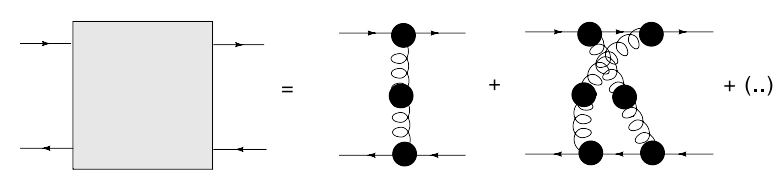

Fig. 20. The four-quark 1PI Green's function and the first terms of its skeleton expansion. 
still goes like $\left(k^{2}\right)^{2 \kappa-1}$. From the first term of the skeleton expansion for the quark-quark scattering kernel one obtains $H\left(k^{2}\right) \sim 1 / k^{2}$. If one would derive a potential for such an interaction one finds

$$
V(\mathbf{r})=\frac{1}{(2 \pi)^{3}} \int \mathrm{d}^{3} p H\left(k^{0}=0, \mathbf{k}\right) \mathrm{e}^{\mathrm{i} \mathbf{k r}}=\frac{1}{(2 \pi)^{3}} \int \mathrm{d}^{3} k \frac{\mathrm{e}^{\mathrm{ikr}}}{\mathbf{k}^{2}} \sim \frac{1}{|\mathbf{r}|},
$$

i.e., the well-known Coulomb potential. (Note, however, that the notion of a potential in chirally symmetric QCD is certainly not appropriate.) As we have seen in Section 3.7, also the resulting running coupling from the quark-gluon vertex is no longer diverging but goes to a fixed point in the infrared similar to the couplings from the Yang-Mills vertices. In a sense, the restoration of chiral symmetry is therefore directly linked with the disappearance of infrared slavery. This is one of the main results of this work.

\subsection{The four-quark Dyson-Schwinger equation}

We now proceed by justifying the above results directly from the Dyson-Schwinger equation for the four-quark 1PI Green's function, which is shown in Fig. 21. This equation describes the full amplitude that includes possible bound states, scattering as well as off-shell effects. In contrast to the skeleton expansion Fig. 20, the diagrams on the right hand side of Fig. 21 all involve a bare quark-gluon vertex. Since this vertex is not infrared-divergent it is therefore not obvious how both equations can give a similar infrared behavior for the four-quark function. Indeed, when considering only the graphs in the first line of Fig. 21 an infrared power counting similar to that in Section 3 yields scaling laws

$$
\left(p^{2}\right)^{2}\left(\left(p^{2}\right)^{-\kappa-1 / 2}\right)^{3}\left(\left(p^{2}\right)^{2 \kappa-1}\right)^{2}=\left(p^{2}\right)^{-3 / 2+\kappa},
$$

independently of the quark kinematics. Here, the second term arises from the three dressed quarkgluon vertices and the third one from the two gluon propagators, whereas the quark propagators proved to be scale invariant. These diagrams therefore cannot be responsible for generating a confining quark-antiquark potential.

However, these diagrams are not the leading ones in the infrared. To see this, consider the last graph in the second line of the DSE, Fig. 21. A skeleton expansion of this graph involves, among others, the two-loop graphs shown in Fig. 22. In complete analogy to the analysis of the second ("t-channel") version of the DSE discussed in Appendix B this two-loop integral has a kinematic region, shown on the left side (a) of Fig. 22, where the vertex correction given by the "small" loop is dominated by hard loop momenta of the order of the quark mass and not by the infrared regime. This graph (a) represents the proper kinematics to study the confining properties of bound states. Here the four-momenta of the massive quarks in a meson are finite and do not scale to zero. However, the exchanged gluon momentum would become soft when the two quarks are sufficiently spatially separated. In this infrared limit the counting for the whole graph involves four dressed quark-gluon vertices in the soft-gluon limit $p \rightarrow 0$ where these vertices are divergent according to our analysis in Section 3.5. This then yields

$$
\left(p^{2}\right)^{2}\left(\left(p^{2}\right)^{-\kappa-1 / 2}\right)^{4}\left(\left(p^{2}\right)^{2 \kappa-1}\right)^{2}=\left(p^{2}\right)^{-2}
$$

i.e., a strong singularity in agreement with the one found in the skeleton expansion. As discussed above, this singularity has the potential to permanently confine quarks.

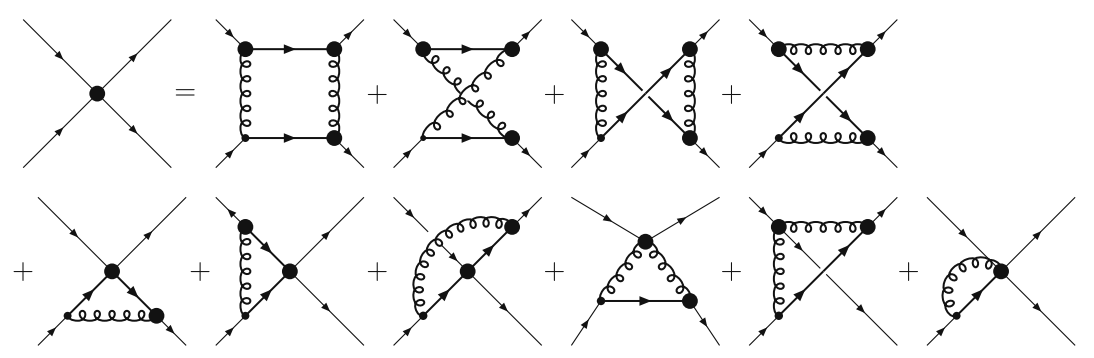

Fig. 21. The DSE for the four-quark 1PI Green's function. 

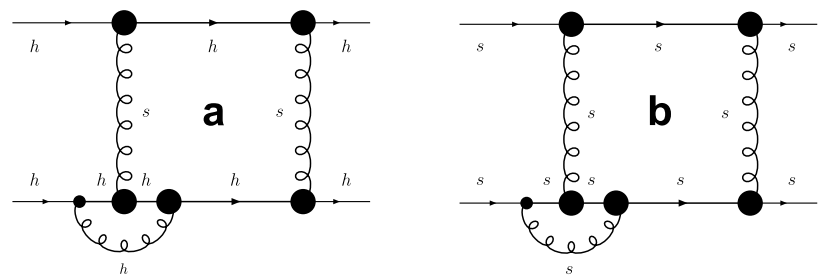

Fig. 22. Contribution to the skeleton expansion of the last term in the four-quark DSE, Fig. 21. Soft and hard momenta are denoted by an $s$, respectively, $h$.

Now consider the right graph (b) were the quark momenta are also soft and scale to zero. In Minkowski space this would correspond to the case of light quarks close to the on-shell limit, i.e., in some sense the opposite case to that where the quenched approximation we consider here is justified. Nevertheless let us study the power-counting in this case. Naively the right graph shown in Fig. 22 seems to yield an equally strong divergence as the preceding one. However, there is no large quark mass here, so that the integral is dominated by small modes. Consequently all dressing functions are probed in their scaling region and it is the uniform limit of the quark-gluon vertices that is relevant here. The resulting power law is similar to the one of Eq. (66), i.e., not sufficiently divergent to trigger a linear rising potential. Comparing to the analysis of diagram (a) above, we see that (i) it is not the uniform infrared limit of the quark-gluon vertex that is responsible for quark confinement but the soft-gluon limit and (ii) it is impossible to confine light quarks close to the on-shell limit with this mechanism, whereas heavy quarks are confined.

Unfortunately the four-quark DSE requires two-loop graphs and is far too involved to be useful for any explicit analysis of heavy bound states. Therefore, in practical calculations one has to resort to the skeleton expansion given in the last subsection. Nevertheless, we wish to emphasize again that the strong interaction between heavy quarks seen in this expansion is indeed also a feature of the underlying functional equation.

\section{Theoretical aspects of confinement}

\subsection{Absence of long range forces}

It is sometimes argued that a strong, confining, quark-quark scattering kernel based on non-perturbative one-gluon exchange is not possible because it gives rise to van der Waals forces between color-singlet hadrons. Confinement, however, implies the absence of these forces. In nature this corresponds to nuclear forces described by an exponentially suppressed potential of the form

$$
V_{11}(R) \propto \mathrm{e}^{-m_{\pi} R}
$$

at medium to large distances. Prominent long range forces also are not observed in quenched lattice simulations [75]. We therefore have to address the question whether the strong quark-quark scattering kernel found in this work necessarily implies power-law suppressed $R^{-\alpha_{s}}$ van der Waals forces between hadrons. As will be argued in the following, this is not the case.

Let us begin with simple examples, building up to the more general case. First note that a single gluon exchange between color-singlet hadrons is forbidden by color algebra. Next consider a twogluon exchange between the two singlets. A corresponding diagram is, e.g., given by

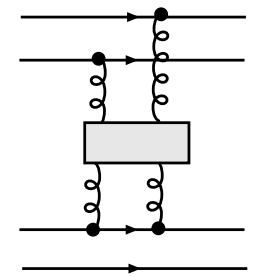


where the two gluons combine to a color-singlet exchange. Such diagrams are non-vanishing and indeed expected to drive pomeron exchange [76]. However, they could only lead to van der Waals forces in the absence of a mass gap, implying a massless glueball in contradiction with lattice gauge theory. Consequently such diagrams should give rise to a Yukawa potential $V_{11} \sim \mathrm{e}^{-M_{G} R}$ in the static limit, which is suppressed at long distances $R$ by the glueball mass $M_{G}$.

One can naturally employ a strong one-gluon exchange mechanism if simultaneously quarks are exchanged to balance color, namely

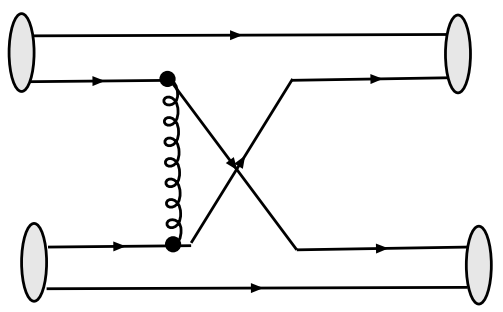

where we have explicitly shown the Bethe-Salpeter amplitudes coupling quarks to the physical hadron singlets. If we evaluate this diagram in the center of mass frame the two quark and gluon vertices and all propagators are evaluated in the small momentum scaling region. As a consequence, the infrared counting would give a power of $\left(p^{2}\right)^{-2}$ from the exchange kernel, which is countered by powers of $\left(p^{2}\right)^{4+4 B S}$ from the two momentum integrations and the Bethe-Salpeter amplitudes. However, these amplitudes are finite in the infrared $(B S=0)$, since the Bethe-Salpeter equation

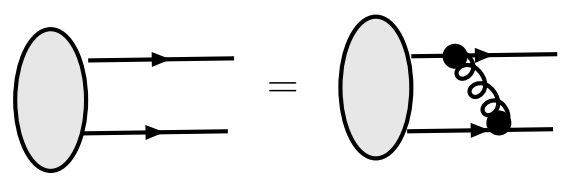

displays a cancellation of the type $\left(p^{2}\right)^{2-2}$ between powers of momenta from the enhanced quarkquark scattering kernel and the internal momentum integration. Finite Bethe-Salpeter amplitudes are also required from physical considerations within the context of core nucleon-nucleon repulsion [77]. We therefore conclude that also the amplitude (70) does not lead to colored van der Waals forces.

This above analysis is quite general. For the purpose of analyzing long-range forces we can limit ourselves to color-singlet exchanges as the only non-vanishing amplitudes. However, in these diagrams the absence of long-range residual forces can be guaranteed by the sufficient condition that all color singlet, full scattering kernels in the theory are gapped, that is, they have no pole with zero mass. This condition is natural in that no massless particle is present in the experimental spectrum. Of course its validity remains to be shown in full Quantum Chromodynamics. Our point here is only that enhanced one-gluon exchange potentials do not necessarily generate van der Waals forces between singlet hadrons. The detailed behavior of the colorsinglet kernels that appear in diagrams like (69) and (70) remain to be investigated in future work.

Finally, it is worth examining the original Hamiltonian argument for long-ranged colored forces given in [78]. One divides the Hilbert space into two sectors, $P$ and $Q$, where $P$ projects over states in which two interacting quark and gluon clusters are in a color-singlet configuration and $Q$ is the part in which the clusters are in non-singlet color configurations (the total state still being a singlet). Then the Hamiltonian can be exactly reduced to the color-singlet $P$ sector as

$$
P H_{\text {eff }}(E) P=P H P+P H Q \frac{1}{E-Q H Q} Q H P .
$$


The presence of an infrared enhanced confining potential $\sigma R$ in the unconfined-color sector $Q H Q$ appears to yield a power-suppressed $P H_{\text {eff }} P \propto 1 / R$ van der Waals potential in the singlet-singlet (confined) sector. However, the correct procedure to obtain non-relativistic inter-hadron interactions from the Landau gauge Green's functions is to perform a non-relativistic reduction not of the quark-quark but of the hadron-hadron scattering kernel. Since the kernel is gapped in the infrared, such a procedure would lead to Yukawa interactions

$$
\frac{1}{k^{2}+M^{2}} \rightarrow \frac{1}{\mathbf{k}^{2}+M^{2}} \rightarrow \mathrm{e}^{-M R}
$$

It is certainly not correct to perform a non-relativistic reduction of a confining quark-quark scattering kernel and then attempt to construct a hadron-hadron interaction Hamiltonian from it. Thus, the formal arguments of Gavela et al. do not apply to our approach.

\section{2. $N$-ality dependence of the string tension}

There are good arguments from both continuum and lattice QCD that the string tension $\sigma$ between static (anti-) quarks follows scaling laws that are dependent on the distance $R$ between the quark and antiquark and the representation of the gauge group. At intermediate distances $R$ the string tension between quarks in the representation $r$ follow the so called Casimir scaling, i.e., $\sigma_{r}=C_{r} / C_{F} \sigma_{F}$, where the subscript $F$ denotes the fundamental representation and $C_{r}, C_{F}$ the respective Casimir operators. At asymptotically large distances, however, the scaling law changes to $\sigma_{r}=f(k) \sigma_{F}$, where $f(k)$ is a function of the $\mathrm{N}$-ality $k$ of the representation $r$ (see $[79,80]$ for reviews).

Casimir scaling is exactly derivable from one-gluon-exchange type forces, thus the first term of the skeleton expansion in Fig. 20 agrees with this behavior. All other diagrams involve quark propagators. From our discussions in Sections 3.8 and 4.3 we recall that for really heavy quarks we find the perturbative mass dependence of the quark-gluon vertex and the propagators implying that all these diagrams are suppressed by powers of the quark mass $M$. Thus, approaching the static limit we recover Casimir scaling in our quark-antiquark potential, as we should [81].

Next we discuss the $\mathrm{N}$-ality dependence of the asymptotic string tension. The $\mathrm{N}$-ality of a given representation is the number of boxes in its Young tableau, modulo N. For SU(3) one has $k=1$ for the fundamental representation (one box for the quark) and $k=0$ for the adjoint representation $(3(\bmod 3)=0)$ for the gluon or octet fermion. In string-type models inspired in lattice gauge theory such as the Flux tube model [82-84], the string is postulated to snap if the hadron may decay to two color-singlet clusters. The energy stored in the flux tube has to exceed the threshold for the hadron-hadron combined energy, and thus this happens only at large distances. It then follows from color algebra that the string between two fundamental sources in a triplet/ anti-triplet representation can never snap in quenched approximation, since sea quarks are absent. Only gluons in the adjoint representation can be produced from the vacuum and these cannot combine with fundamental quarks to yield a color-singlet hadron. However, the octet-octet string can break, via two-gluon production, one of them combining with each source to make a color singlet. Thus, it is apparent that the string tension indeed scales with the $\mathrm{N}$-ality of the representation.

It is instructive to discuss how this works in the Green's functions approach to Landau gauge QCD. Certainly, the quark-quark scattering kernel with one gluon exchange cannot be responsible for this type of behavior, since it also confines fermions in the adjoint representation, leading to Casimir scaling. However, this is only a four-point Green's function and by far not the only contribution to the computation of the string tension, say, via the Wilson loop. The following two graphs represent the first skeleton diagram of the 6-point Green's function that make N-ality scaling conceivable. The left diagram corresponds to fermions in the adjoint representation, the right diagram in the fundamental representation. 

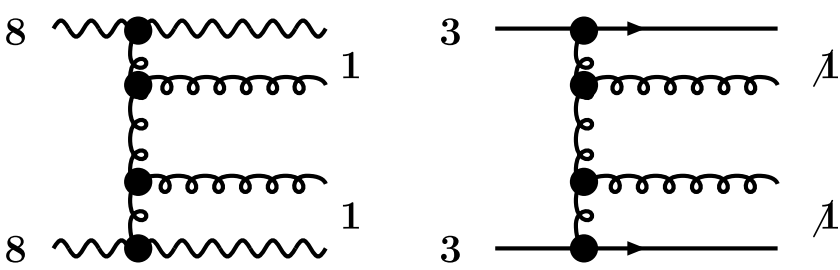

If we send all scales simultaneously to zero as $P \rightarrow 0$, both diagrams are strongly infrared enhanced and with the help of our counting we establish a power law $\left(P^{2}\right)^{-3-2 \kappa}$. However, in this case, all colored particles are simultaneously being separated, and we would still expect confinement to be at work. If now we separate the two clusters, keeping a finite momentum between the components of each cluster, most vertices and propagators have finite momenta running through, and only the middle gluon rung contributes to an infrared exponent of $\left(p^{2}\right)^{-1+2 \kappa}$, therefore being suppressed.

It is obvious that a full evaluation of the string tension from the Wilson loop requires the interplay of many such higher Green's functions, with complicated color structure. The interplay of these to yield the correct $\mathrm{N}$-ality is a complete other project in itself. However, it is obvious from these comments that demanding $\mathrm{N}$-ality to arise from our quark-quark scattering kernel alone is not appropriate. It should (as it correctly does) yield Casimir scaling.

\section{Phenomenological aspects of confinement}

\subsection{Kernel non-relativistic reduction}

We would now like to examine the limit of our quark-quark scattering kernel when the quark mass is large and see how close we can get to a Schrödinger description. Before we proceed, let us remark that within full QCD a description of the quark-antiquark interaction in terms of a time independent potential is not entirely adequate [85-87]. ${ }^{9}$ The time-independent Schrödinger equation following from Coulomb-gauge QCD would be formulated in a Fock space with explicit transverse gluons. However, considering the strict non-relativistic reduction of the quark-quark scattering kernel in Landau gauge, i.e., seeking a Schrödinger equation for a two-body problem, we need to insist on the elimination of all gluonic degrees of freedom. This then leads to a strongly energy-dependent kernel, which is equivalent to a time-dependent potential in coordinate space.

Nevertheless it is instructive to go through the exercise, since there may be a window of quark masses where a potential could be of use (while certainly important effects from virtual states with transverse gluons are expected too). This allows us to make contact with time-honored phenomenological and lattice computations in the static limit.

\subsection{Salpeter equation}

The main problem in deriving a Salpeter equation for a bound meson state are energy singularities in the loop momentum $k$ of the Bethe-Salpeter equation. In QED, ladder and cross-ladder diagrams in the Bethe-Salpeter kernel conspire to cancel these singularities, as detailed in Appendix I. However, due to factors from the color algebra such cancellations are unlikely to also occur in QCD. If one insists on maintaining the simplicity of the ladder approximation one therefore faces singularities in $k_{0}$ from both the quark propagators and the kernel (gluon propagators and vertices). In non-relativistic reduc-

\footnotetext{
${ }^{9}$ One argument is: within such a description the extension of a heavy meson at fixed radial quantum number $n_{r}$ would become vanishingly small in the static limit, i.e., $\langle r\rangle_{n_{r}} \rightarrow 0$, and see exclusively the Coulomb part of the potential. This part being scaleless all Balmer splittings $m_{n_{r+1}}-m_{n_{r}}$ would scale with the quark mass $M$. However, the glueball masses $m_{G}$ would not. So the states neighboring any one $n_{r}$ heavy quarkonium state would not be the corresponding $n_{r \pm 1}$ state, but instead a state with mass $m_{n_{r}}+m_{G}$. We thank Gunnar Bali for this observation.
} 
tions it has been common practice [88-91], to ignore the $k_{0}$ dependence of the kernel and Bethe-Salpeter wavefunctions and perform the pole integrals by putting only the intermediate quarks on-shell. The Bethe-Salpeter equation is thus inconsistently evaluated with $k_{0}=0$ for the kernel and $k_{0} \neq 0$ for the propagating quarks. This procedure is justified empirically by demanding finite support for the BS-amplitude when the quarks are off-shell.

The resulting Salpeter equation has many nice features such as color confinement (by sending colored objects to infinite mass outside the spectrum) in the presence of infrared enhancement kernel. It also respects chiral symmetry and it can be obtained as a collective excitation (Random Phase Approximation) in a Hamiltonian framework [92,93].

In the following we go through the derivation of the Salpeter equation and discuss how it can be used to generate a static potential from our relativistic quark-quark interaction kernel. Here we follow Bicudo and Ribeiro [89] (see also Itzykson and Zuber, [71], for an analogous derivation without propagator dressing functions) by defining directly a positive (negative) Salpeter function from the BetheSalpeter amplitudes to emit (absorb) a quark-antiquark pair from the meson, evaluated at zero energy and integrated over the propagators

$$
\phi^{+}(\mathbf{k}) \equiv \int \frac{\mathrm{d} k_{0}}{2 \pi} S_{q}\left(\mathbf{k}, P_{0} / 2+k_{0}\right) S_{\bar{q}}\left(-\mathbf{k}, P_{0} / 2-k_{0}\right) \phi^{+}(\mathbf{k}, 0)
$$

(and analogously for $\phi^{-}$). The Bethe-Salpeter equations then reduce to the coupled Salpeter equations for the two amplitudes $\phi^{ \pm}$, the first of which becomes, now including the spin index,

$$
\begin{aligned}
\phi^{+}(\mathbf{k})_{\lambda_{1}, \lambda_{2}}= & {\left[\int \frac{\mathrm{d} k_{0}}{2 \pi} S_{q}\left(\mathbf{k}, P_{0} / 2+k_{0}\right) S_{\bar{q}}\left(-\mathbf{k}, P_{0} / 2-k_{0}\right)\right] } \\
& \times\left(\int \frac{\mathrm{d} \mathbf{k}^{\prime}}{(2 \pi)^{3}} u_{i}^{\dagger}\left(\mathbf{k}, \lambda_{1}\right) v_{n}^{\dagger}\left(-\mathbf{k}^{\prime}, \lambda_{4}\right) K_{i j m n}\left(\mathbf{k}-\mathbf{k}^{\prime}\right) v_{j}\left(-\mathbf{k}, \lambda_{2}\right) u_{m}\left(\mathbf{k}^{\prime}, \lambda_{3}\right) \phi^{+}\left(\mathbf{k}^{\prime}\right)_{\lambda_{3}, \lambda_{4}}\right. \\
& \left.-\int \frac{\mathrm{d} \mathbf{k}^{\prime}}{(2 \pi)^{3}} u_{i}^{\dagger}\left(\mathbf{k}, \lambda_{1}\right) u_{n}^{\dagger}\left(-\mathbf{k}^{\prime}, \lambda_{3}\right) K_{i j m n}\left(\mathbf{k}-\mathbf{k}^{\prime}\right) v_{j}\left(-\mathbf{k}, \lambda_{2}\right) v_{m}\left(-\mathbf{k}^{\prime}, \lambda_{3}\right) \phi^{-}\left(\mathbf{k}^{\prime}\right)_{\lambda_{3}, \lambda_{4}}\right),
\end{aligned}
$$

where the interaction part can be represented graphically with an instantaneous Bethe-Goldstone diagram (note fermions and anti-fermions are distinguished, anti-fermion always the bottom line)

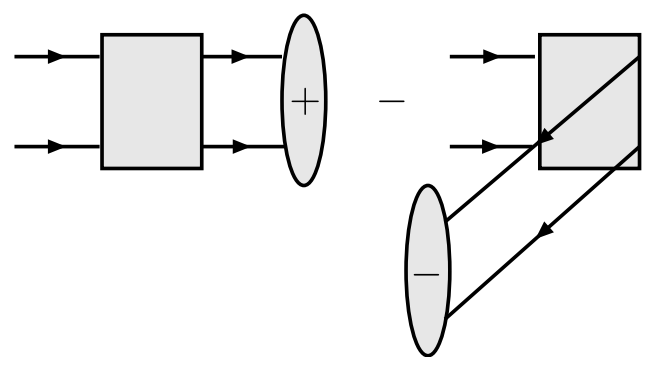

The integral over the propagators in the first line yields

$$
\frac{1}{M-E_{q}-E_{\bar{q}}}
$$

and puts the quarks "on-shell". One should note however that in the presence of a strong kernel, $E_{q}=\sqrt{A^{2}(\mathbf{q}) \mathbf{q}^{2}+B^{2}(\mathbf{q})}$ is actually divergent $[89,94]$ (when the same approximations are applied to the propagator Dyson-Schwinger equation) and these divergences in the self-energies cancel only for color-singlet states. 
To take the strict static limit one neglects $\phi^{-} \ll \phi^{+}$(this approximation is equivalent to TammDancoff and breaks chiral symmetry). Then Fourier transforming to coordinate space by means of

$$
V(r)=\int \frac{\mathrm{d} \mathbf{q}}{(2 \pi)^{3}} V(q) \mathrm{e}^{\mathrm{i} \mathbf{q r}}
$$

and

$$
E_{i}(r)=\int \frac{\mathrm{d} \mathbf{q}}{(2 \pi)^{3}} \mathrm{e}^{\mathrm{i} \mathbf{q r}}\left(E_{i}(q)-E_{i}(0)\right)
$$

one finally obtains a Schrödinger-type equation (the infrared cancellation for color singlets is written explicitly)

$$
M \psi(r)=\int \mathrm{d}^{3} \mathbf{r}^{\prime}\left[E_{q}\left(\mathbf{r}-\mathbf{r}^{\prime}\right)+E_{\bar{q}}\left(\mathbf{r}-\mathbf{r}^{\prime}\right)\right] \psi\left(r^{\prime}\right)+\left[V(r)-E_{q}(\mathbf{q}=0)-E_{\bar{q}}(\mathbf{q}=0)\right] \psi(r) .
$$

The potential $V(r)$ in this equation will be considered further in the next subsection.

The insight one gains from the Salpeter construction so devised is that the static potential obtained from a Born approximation is correct (modulo the infrared subtraction as needed for bound state equations with self-energies included). ${ }^{10}$ Thus, one can test the quark-quark kernel constructed in Landau gauge QCD by evaluating the static potential in the Salpeter approximation. However, it is difficult to estimate the systematic error of this procedure. One way to estimate this is to cross-check with the Gross approximation, detailed in Appendix I.2. Relativistic corrections to the potential of $O\left(v^{2}\right)$ could be investigated by appropriately expanding the spinors contracting the kernel and employing a Fierz rearrangement. These could also be evaluated in the Salpeter approach and compared to results from the lattice [85]. In general, the systematic error of the Salpeter equation is controllable in $1 / M$ if one can independently establish that the BS wave amplitude has limited support in the quark off-shellness. ${ }^{11}$

\subsection{Salpeter string tension}

We are now using the results of the last subsection to relate phenomenology with the fundamental building blocks of our quark-quark scattering kernel, i.e., the quark-gluon vertex and the gluon propagator. To this end we will take the limit of our kernel with the quark legs going on-shell, yielding $q_{0}=0$ for the exchanged energy (ignoring possible singularities of the amputated kernel). The resulting non-relativistic potential is the appropriate one to be used in the context of the Salpeter (RPA) equation and is known to be about $\sigma=\frac{3}{4} 0.18 \mathrm{GeV}^{2}[92,95]$.

We denote the momenta of the incoming quarks by $p_{1}, k_{1}$ and the outgoing $p_{2}, k_{2}$. The momentum transferred by the gluon rung is $q$. We choose the center of mass frame, where $\mathbf{p}_{1}=-\mathbf{k}_{1}$. We let $W$ be the total energy and $p_{1}^{0}=W / 2+E / 2, k_{1}^{0}=W / 2-E / 2$ the energy of the two incoming quarks ( $E$ ' for the outgoing). From the on-shell conditions for the quarks one finds that $E=E^{\prime}=0$ in the center of mass frame. The gluon momentum is given by $q=(0, \mathbf{q})$.

$$
p_{1}=\left(\frac{W}{2}, \mathbf{p}_{\mathbf{1}}\right)
$$

The one-rung skeleton amputated kernel (77) then becomes

\footnotetext{
${ }^{10}$ Moreover, one learns that in deriving Eq. (76) a collective mode, namely the back-propagating Salpeter wavefunction, has been neglected. Taking this mode into account gives rise to additional energy-dependent hyperfine-terms proportional to $1 / M^{2}$ in the non-relativistic Schrödinger equation.

11 We thank A. Szczepaniak for calling our attention to this point.
} 


$$
K_{\mathrm{IR}}=g^{2} C_{F} \sum_{i, j=1}^{12} \Gamma_{i}^{\mu}\left(p_{1}, p_{2}\right) \lambda_{i}\left(M^{2}, \mathbf{q}^{2}\right) D_{\mu v}(q) \Gamma_{j}^{v}\left(k_{1}, k_{2}\right) \lambda_{j}\left(M^{2}, \mathbf{q}^{2}\right),
$$

where the Casimir $C_{F}=\frac{N^{2}-1}{2 N}$ stems from the color trace and the quark-gluon vertex is denoted by $\Gamma_{\mu}=\sum_{i=1}^{12} \Gamma_{i}^{\mu} \lambda_{i}$.

Next we contract each vertex of the kernel with external spinor wavefunctions $\left(u^{\dagger} \Gamma^{\mu} u\right) / Z_{2}$ (should the kernel be part of a loop, the propagator attached to the right vertices needs to be decomposed as $S=u u^{\dagger} /\left(D_{+}\right)+v v^{\dagger} /\left(D_{-}\right)$, and we keep the positive energy pole). Note that the spinors need to be multiplied with the inverse of the wavefunction renormalization constant $Z_{2}$ in order to make the construction renormalization-group invariant. With the intent of obtaining the central potential we average over initial and sum over final spins. This means that at each vertex we have $\frac{1}{2} \sum_{\lambda_{1} \lambda_{2}} \operatorname{Tr}\left(u_{\lambda_{2}} u_{\lambda_{1}}^{\dagger} \Gamma^{\mu}\right) / Z_{2}$. In the extreme non-relativistic limit, the $1 / M$ lower components vanish. In terms of Pauli bispinors we are then left with

$$
\frac{1}{2} \sum_{\lambda_{1} \lambda_{2}}\left(\begin{array}{c}
\chi_{\lambda_{2}} \\
0
\end{array}\right)\left(\begin{array}{ll}
\chi_{\lambda_{1}}^{\dagger} & 0
\end{array}\right)=\frac{1}{2}\left(\begin{array}{llll}
1 & 1 & 0 & 0 \\
1 & 1 & 0 & 0 \\
0 & 0 & 0 & 0 \\
0 & 0 & 0 & 0
\end{array}\right) \frac{1}{2}\left(\frac{\square+\gamma_{0}}{2}\right)\left(\square+\sigma^{y z}\right) .
$$

Herein, we have made a choice of quantization axis for the spin so that rotational invariance is no more manifest. If necessary this can be avoided by employing a generic vector $\hat{n}$. Plugging this spinor combination into Eq. (78) and tracing each of the two vertices (with 12 tensors each) is conveniently carried out with the help of a symbol manipulation program such as FORM [96]. We furthermore define $p_{1} \equiv p$ and perform the Wick-rotation to Euclidean space.

Employing momentum conservation, $k_{2}=k_{1}+q, p_{2}=p_{1}-q$, only terms with the four "longitudinal" $\lambda_{i}$ pieces remain, with all transverse tensors $\tau_{i}$ contributing to subleading parts of the potential. For the $\lambda_{i}$ we may write

$$
\lambda_{i}\left(M^{2}, M^{2}, q^{2}\right)=A_{i}\left(q^{2}\right)^{-1 / 2-\kappa},
$$

using our results from previous sections. After some algebra we are left with

$$
\begin{aligned}
K_{\mathrm{IR}}^{\prime}= & \frac{g^{2} C_{F} Z}{q^{4}\left(Z_{2}\right)^{2}}\left(A_{1}^{2}+2 A_{1} W\left(A_{2}-A_{3}\right)+4\left(W A_{2}-A_{3}\right)^{2}\left(4(\mathbf{p} \cdot \hat{\mathbf{q}})^{2}-\mathbf{p}^{2}-\frac{W^{2}}{4}\right)\right. \\
& \left.-4 A_{4}^{2}\left((\hat{\mathbf{q}} \times \mathbf{p})_{x}^{2}-\left(\mathbf{p}^{2}-p_{x}^{2}\right)\right)\right),
\end{aligned}
$$

where the factor $Z$ is the coefficient of the power law $Z\left(p^{2}\right)=Z \times\left(p^{2}\right)^{2 \kappa}$ of the gluon propagator in the infrared. Note that the total energy $W \sim M$ and $p \propto 1 / M$ such that the last term is suppressed for large quark masses and the frame dependence disappears from the central potential. To extract the central, static potential in the center of mass frame, we keep the first three terms and take $W=2 M$. Comparing (80) to the Fourier transform of a linear potential $\sigma r \rightarrow-8 \pi \sigma / q^{4}$, we read off

$$
\sigma=\frac{\alpha_{s} Z}{2\left(Z_{2}\right)^{2}}\left(A_{1}^{2}+4 M A_{1}\left(2 M A_{2}-A_{3}\right)-4 M^{2}\left(2 M A_{2}-A_{3}\right)^{2}\right)
$$

and $\alpha_{s}=g^{2} / 4 \pi$.

This string tension has to match the one extracted from the quarkonium static potential, $\sigma_{\exp }=(0.424 \mathrm{GeV})^{2} . Z_{2}$ is very close to 1 and $Z \simeq 20(\mathrm{GeV})^{-4 \kappa}$ can be read-off the solution for the vacuum polarization DSE, with a renormalization point $\mu \simeq 13 \mathrm{GeV}$, such that $\alpha_{s}(\mu) \simeq 0.5$. Ignoring $A_{2}$ and $A_{3}$, we then have a phenomenological estimate $A_{1} \simeq 0.16 \mathrm{GeV}^{1+2 \kappa}$ where the energy units are GeV but the precise mass dimension depends on $\kappa$, and is here fixed from the gluon propagator $Z$ to $\kappa=0.595$.

Of course, this number is just an order-of-magnitude guess, since the string tension obtained from the quark-quark 2PI needs to be obtained by summing a skeleton series as previously discussed in length. The second term in the skeleton is the crossed-rung, one-loop diagram that causes a correction to the one-gluon string tension 


$$
\sigma \simeq \sigma^{1 g}\left(1-\frac{1}{6} \frac{\alpha_{s} A_{1}^{2} Z}{M^{2}} c\right)
$$

where $c$ is a pure number containing integration factorials, phase space and angular factor. We explicitly show the color factor, and $M$ is a quark mass scale showing convergence of the skeleton expansion in its presence. The corrections to the string tension can be organized as

$$
\sigma \simeq \sigma^{1 g}\left(1-c_{1} \frac{\sigma^{1 g}}{M^{2}}+\cdots\right)
$$

To conclude this subsection, we note in passing that in the light of our calculation the longstanding discussion on whether confinement is of a vector $\gamma \gamma$ or of a scalar nature seems oversimplified. Each of the quark-gluon vertices in our expansion of the quark-quark scattering kernel contains a full set of 12 Dirac tensors with vector, scalar and tensor structures. After contraction with the gluon propagator one obtains a large number of terms and non-trivial cancellations can arise. The detailed structure of the resulting quark-anti-quark interaction needs to be investigated further and is certainly of great interest due to recent improved lattice data [97].

\subsection{Mass dependence of the string tension}

In this subsection we consider the possible mass dependence on the string tension from a phenomenological point of view. To this end it seems sufficient to consider the non-relativistic $q \bar{q}$ Schrödinger equation, which contains some of the features exploited in more elaborate schemes as for example pNRQCD [98]. The reduced radial wave function $u(r)$ satisfies

$$
\frac{\hbar^{2}}{2 \mu} u^{\prime \prime}(r)+\left[E-\lambda r^{v}-\frac{l(l+1) h^{2}}{2 \mu r^{2}}\right] u(r)=0
$$

with a power-law potential $V(r)=\lambda r^{v}$ and the reduced quark mass $\mu=M_{Q} / 2$. In particular, $v=1$ and $\lambda=-\sigma$ corresponds to the linear potential, $v=-1$ and $\lambda=\alpha_{s}$ to the (attractive) Coulomb potential, and the limit $v \rightarrow 0$ can be used to represent a logarithmic confining potential. The equation can be transformed to a scaleless form [99,100] by performing the change of variables $(r, E) \rightarrow(\rho, \varepsilon)$ :

$$
r=\rho\left(\frac{h^{2}}{2 \mu|\lambda|}\right)^{\frac{1}{2+v}}, \quad E=\varepsilon\left(\frac{h^{2}}{2 \mu|\lambda|}\right)^{\frac{-2}{2+v}} \frac{h^{2}}{2 \mu} .
$$

Therefore, all the mass and parameter scaling of the spectrum and momenta (inverse length) needs to reside in Eq. (85). For the Coulomb potential $v=-1$ one obtains

$$
p \propto \mu \alpha_{s}, \quad E \propto \mu \alpha_{s}^{2},
$$

stating that all splittings grow with the quark mass at large $M_{Q}$ since the (low-lying) states become smaller and see mostly the short-range Coulomb potential of pQCD. A linear potential, however, causes splittings slowly decreasing with the quark mass and growing with the string tension

$$
p \propto(\mu \sigma)^{1 / 3}, \quad E \propto \mu^{-1 / 3} \sigma^{2 / 3} .
$$

In Fig. 23 we show splittings between the $2 s$ and $3 s$ quarkonia for $\Upsilon, \psi, \phi$ and $\rho \pi$ systems. Ignoring $s-d$ wave mixing a typical assignment for these states are $\Upsilon(10355), \Upsilon(10023), \psi(4040), \psi(3686)$, $\phi(2170), \phi(1680)$. There is no candidate for $I=0 \omega(3 s)$ so we give instead the $I=1 \pi, \rho$ splitting using $\rho(1900), \rho(1465), \pi(1800), \pi(1300)$. However, one has to bear in mind that large errors in the mass determination and the general failure of a non-relativistic quark model for light quarks make these splittings less useful. The thick line in our plot represents a scaling based on Eq. (85) where a mass independent string tension is assumed, i.e., $v=1$.

With respect to these data we now discuss whether a strong mass dependence of the string tension is possible. The $\phi$ and $\psi$ states fall nicely on the $\mu^{-1 / 3}$ dependence suggested by a linear 


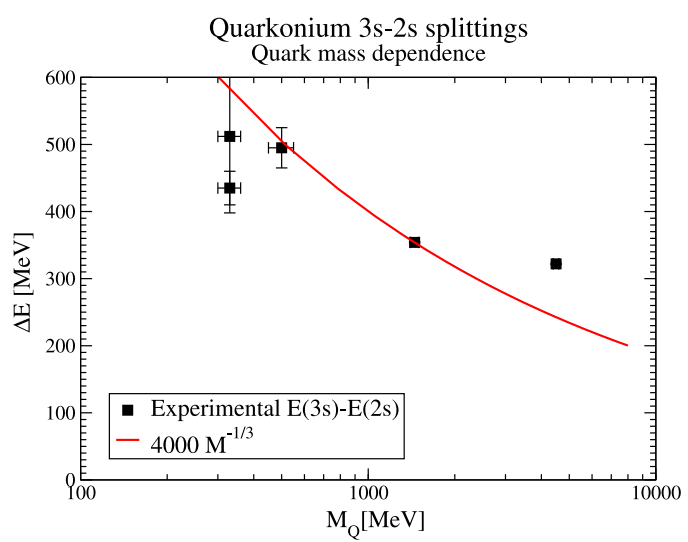

\begin{tabular}{|cc|cc|}
\hline Charmonium & $E-E_{J / \psi}$ & Bottomonium & $E-E_{\Upsilon}$ \\
\hline$\eta_{c}$ & -117 & $\eta_{b}$ & unknown \\
$J / \psi$ & 0 & $\Upsilon$ & 0 \\
$\chi_{c 0}(1 p)$ & 318 & $\chi_{b 0}(1 p)$ & 399 \\
$\chi_{c 1}(1 p)$ & 414 & $\chi_{b 1}(1 p)$ & 433 \\
$h_{c}$ & 429 & $h_{b}$ & unknown \\
$\chi_{c 2}(1 p)$ & 459 & $\chi_{b 2}(1 p)$ & 452 \\
\hline$\eta_{c}(2 s)$ & $540(4)$ & $\eta_{b}(2 s)$ & unknown \\
$\psi(2 s)$ & 589 & $\Upsilon(2 s)$ & 563 \\
$\psi(3770)$ & 675 & $\Upsilon(1 d)$ & 701 \\
$X(3872)$ & 768 & No equivalent & known \\
$\chi_{c 2}(2 p)$ & 832 & $\chi_{b 2}(2 p)$ & 809 \\
$X(3940)$ & $843(5)$ & $\chi_{b 0}(2 p)$ & 772 \\
$X(3945)$ & $848(11)$ & $\chi_{b 0}(2 p)$ & 795 \\
\hline$\psi(4040)$ & $942(1)$ & $\Upsilon(3 s)$ & 895 \\
$\psi(4160)$ & $1056(3)$ & $\Upsilon(2 d)$ & unknown \\
$\psi(4260)$ & $1166(12)$ & $\Upsilon(4 s)$ & 1119 \\
$\psi(4320)$ & 1223 & $\Upsilon(3 d)$ & unknown \\
$\psi(4415)$ & $1324(4)$ & $\Upsilon(5 s)$ & 1405 \\
$\psi(4660)$ & 1563 & $\Upsilon(4 d)$ & $1559(8)$ \\
\hline
\end{tabular}

Fig. 23. Left diagram: the dependence of the splitting $E(3 s)-E(2 s)$ on the quark mass. The experimental assignments for $u \bar{u}-$, $s \bar{s}-, c \bar{c}$ - and $b \bar{b}$-states are educated guesses based on the non-relativistic quark model. The thick line represents a scaling based on Eq. (85) where a mass independent string tension is assumed, i.e., $v=1$. Right table: charmonium and bottomonium spectra (quoted are the splittings to the $J / \psi(3097)$ and $\Upsilon(9460)$ ). Data from [58], with new states reported from [101-104].

potential with a mass-independent string tension (thick line). However, as also noted, e.g., in [105], charmonium and bottomonium have a congruent spectrum, i.e., the splittings are almost the same in both systems. This suggests either (i) a logarithmic potential, (ii) a soft mass-dependence of the string tension of a linear potential of the type $\sigma \propto \sqrt{M_{Q}}$, (iii) the beginning of a transition to the Coulomb regime around the $\Upsilon$.

On the other hand, our analytical analysis of the mass dependence of the quark-gluon vertex in Section 3.8 yielded three possibilities: either the vertex-dressing is proportional to $M_{Q}$ which results in $\sigma \propto M_{Q}^{2}$, or it is proportional to $1 / M_{Q}$, yielding $\sigma \propto M_{Q}^{-2}$, or it is independent of the quark mass which gives $\sigma \propto M_{Q}^{0}$. The first case together with Eq. (87) would give a Coulomb-like behavior $E \propto M_{Q}$ that is clearly ruled out by the data. The decreasing self-consistent solution $\sigma \propto 1 / M_{Q}^{2}$ would give $E \propto M_{Q}^{-5 / 3}$ that is too strong a decrease for the data.

On the other hand, the third solution has a quark-mass-independent string tension, scaling as the square of the QCD scale, just like in pure Yang-Mills theories [106]. This behavior could explain the experimental splittings if the Coulomb potential has a large effect in the bottomonium spectrum. It would certainly be desirable to confirm this behavior in a lattice computation of the dependence of the string tension with the quark mass.

Moreover, many of the experimental splittings given in the table in Fig. 23 agree with a quark mass-independent scaling, including many of the exciting new states. Of course, in the absence of coupled open decay channels one would like to make the comparison for the highest possible states, since the linear potential would then clearly dominate, but the open channels make the loss of precision high in the spectrum comparable to the loss of precision due to the Coulomb potential for low-lying states. Also, some of the states could be hidden exotics in nature, that is, composed of wavefunctions $q \bar{q} g, q \bar{q} q \bar{q}$ and one needs careful on-resonance data to be able to apply the Franck-Condon principle to distinguish them $[107,108]$.

\section{Summary and conclusions}

In this work we performed a thorough investigation of dynamical chiral symmetry breaking in the quark-gluon interaction of quenched Landau gauge QCD. We considered the coupled dynamical system of Dyson-Schwinger equations for the quark propagator and the quark-gluon vertex and deter- 
mined self-consistent solutions both analytically and numerically. We identified the infrared leading diagrams in this equation and found these to agree with the leading ones in an $1 / N_{c}$ counting scheme. This result is alternative to a number of treatments of the vertex-DSE (see e.g., [15,16,40,41]), that picked out the subleading, Abelian parts of the vertex-DSE. The most important results from our self-consistent treatment are

- The qqg vertex uniform singularities have, in a milder form, been proposed in the past, in BallChiu-type constructions based on the Slavnov-Taylor Identities. We now find an overall (all external scales go to zero) as well as a collinear (the external gluon momentum goes to zero) infrared divergence of the vertex scaling with $\left(p^{2}\right)^{-1 / 2-\kappa}$, provided we take into account dynamical chiral symmetry breaking. This includes corresponding chirally non-symmetric tensor structures in the quark propagator and the quark-gluon vertex, as e.g., Dirac scalars. In our numerical analysis we find a scalar part of the vertex which is of the same strength in the infrared as the conventional $\gamma_{\mu}$-part.

- This infrared divergence implies an infrared-divergent running coupling (defined from the quarkgluon vertex) in contrast to the infrared-finite fixed point behaviors of the couplings in the YangMills sector of the theory. Our findings agree with the old notion of infrared slavery [1], although the mechanism at work is different. In early works it was believed that infrared slavery is caused by an infrared-divergent gluon propagator. In contrast we find an infrared vanishing (or finite) gluon, that is accompanied by an infrared-divergent quark-gluon vertex. Both quantities together drive the coupling to infinity in the zero momentum limit. ${ }^{12}$

- The collinear infrared divergence also triggers an infrared-divergent one-gluon exchange kernel between two quarks, leading to a linear rising static quark potential which implies quark confinement.

- If chiral symmetry is restored by hand, i.e., if the chiral-symmetry-breaking tensor structures in the quark propagator and the quark-gluon vertex are arbitrarily set to zero, we find a different self-consistent solution of the quark propagator and vertex-DSEs: the vertex then has an infrared singularity of order $\left(p^{2}\right)^{-\kappa}$ which is reduced in strength compared to the chirally broken case.

- This reduced infrared strength in the chirally symmetric case leads an infrared constant coupling from the quark-gluon vertex (i.e., similar to the couplings in the Yang-Mills sector) and to a Coulomb-type static quark-antiquark potential, which is not confining. We have therefore uncovered a mechanism which directly links confinement to dynamical chiral symmetry breaking and vice versa.

These are the rigorous results reported in this paper. We have also, in a less rigorous but nevertheless meaningful way, discussed some aspects and consequences of our findings. In particular we gave a prescription to construct a confining and chiral symmetry preserving BetheSalpeter kernel, which includes the infrared divergences found in our analysis. We discussed the mass dependence of the resulting string tension in our approach and found a surprising behavior for the quark mass functions: taking all tensor structures of the quark-gluon vertex into account it seems that the infrared results for the quark mass $M\left(p^{2}=0\right)$ are much less sensitive to the current quark mass than anticipated previously. Whereas there is a change of $O\left(10^{3}\right)$ in the current quark mass from the up- to the bottom quark, we only find changes of a factor 2-3 in $M\left(p^{2}=0\right)$ (somewhat larger changes could be expected in different truncations). As a result we find a string tension which has no simple scaling law with respect to the quark mass. This result seems to be consistent with phenomenological expectations from the splittings in quarkonia.

Finally, we discussed some aspects of the confining nature of our result. We presented arguments in favor of the absence of long-ranged van der Waals forces even in the presence of a strong singularity, such as the one suggested, in the quark-gluon interaction. In a nutshell, the

\footnotetext{
${ }^{12}$ Certainly, there is no Landau pole at intermediate momenta in a non-perturbative treatment of the running coupling. We do find a plateau at mid-to-low momentum, although in a model-dependent manner.
} 
reason here is the absence of color-non-singlet exchanges between color-singlet objects. The remaining color-singlet exchanges then always are gapped such that the interaction vanishes at low momenta. This issue deserves further studies in the future. In addition we shortly discussed the Casimir scaling property of one-gluon exchange and potential candidates of Green's functions which should lead to the $\mathrm{N}$-ality behavior of the string tension at large distances in unquenched QCD.

Finally, it is hard to see how the picture of confinement via a static linear potential may be also adequate for light quarks (see for example [109]). In this respect it is reassuring that we find positivity violations for the light quark propagator, which in turn is a sufficient condition for the confinement of light quarks. At the current technical level of treating the DSE of the quark-gluon vertex this statement is truncation dependent. It remains to be seen, whether this finding can be corroborated in a truncation independent way.

In this work we gave a qualitative picture of what could be a confinement mechanism for quarks in Landau gauge QCD. Although we tried to make our arguments as rigorous as possible, much work remains to be done. In particular it remains to be shown that the quantitative aspects of the picture match expectations from phenomenology. Here it seems desirable to develop a calculational scheme to determine the quark-antiquark interaction in quantitative detail and confront the results with the experimental findings as, e.g., the spectra of charmonia and bottomonia.

\section{Acknowledgements}

The observation that the leading diagram in the vertex Dyson-Schwinger equation is the non-Abelian piece of Eq. (C1) surfaced in conversations with Mandar Bhagwat, Craig Roberts and Peter Tandy. We have enjoyed discussion with many other colleagues such as Gunnar Bali, Stanley Brodsky, Jeff Greensite, Kurt Langfeld, Stefan Olejnik, Jan Pawlowski, Hugo Reinhardt, Lorenz von Smekal, Adam Szczepaniak, Daniel Zwanziger, and several graduate students for the extended duration of this project. We thank Richard Williams for a critical reading of the manuscript. F. J. Llanes-Estrada thanks the members of the institutes of theoretical physics at Tübingen and Graz, and the Institute for Nuclear Physics at Darmstadt for their hospitality and acknowledges travel support from the DAAD, Univ. Complutense, Ministerio de Educacion y Ciencia and project Grants FPA 2004-02602, 2005-02327, PR27/05-13955-BSCH (Spain). This work was also supported in part by Grant M979-N16 of the Austrian Research Foundation FWF, by the DFG under contract AL 279/5-1\&2 and by the Helmholtz-University Young Investigator Grant VH-NG-332.

\section{Appendix A. Structure of the quark-gluon vertex}

The quark-gluon vertex is in general a function of two independent four-momenta as well as its Lorentz and color indices. The tensor decomposition expresses it in a finite basis of the internal spaces given in Section A.1. This also reduces the momentum dependence of the form factors to three independent momentum squares. We will study several different kinematics defined in Section A.2.

\section{A.1. Tensor basis}

In Landau gauge the quark-gluon vertex has 12 different tensor structures conveniently written as

$$
\Gamma^{\mu}=\sum_{i=1}^{4} \lambda_{i} L_{i}^{\mu}+\sum_{i=1}^{8} \tau_{i} T_{i}^{\mu},
$$

where the $T_{i}$ denote parts transverse to the gluon momentum. The general basis for the quark-gluon vertex we employ here has been given by Ball and Chiu [24]. It reads 


$$
\begin{aligned}
& L_{1}^{\mu}=\gamma^{\mu} \\
& L_{2}^{\mu}=-\left(\not p_{1}+\not p_{2}\right)\left(p_{1}+p_{2}\right)^{\mu} \\
& L_{3}^{\mu}=-\mathrm{i}\left(p_{1}+p_{2}\right)^{\mu} \\
& L_{4}^{\mu}=-\mathrm{i} \sigma^{\mu v}\left(p_{1}+p_{2}\right)_{v} \\
& T_{1}^{\mu}=\mathrm{i}\left(p_{1}^{\mu} p_{2} \cdot p_{3}-p_{2}^{\mu} p_{1} \cdot p_{3}\right) \\
& \left.T_{2}^{\mu}=\left(p_{1}^{\mu} p_{2} \cdot p_{3}-p_{2}^{\mu} p_{1} \cdot p_{3}\right)\left(\not p_{1}+\not p_{2}\right)\right) \\
& T_{3}^{\mu}=\not p_{3} p_{3}^{\mu}-p_{3}^{2} \gamma^{\mu} \\
& T_{4}^{\mu}=-\mathrm{i}\left(p_{3}^{2} \sigma^{\mu v}\left(p_{1}+p_{2}\right)_{v}+2 p_{3}^{\mu} \sigma_{\lambda v} p_{1}^{\lambda} p_{2}^{v}\right) \\
& T_{5}^{\mu}=\mathrm{i} \sigma^{\mu v}\left(p_{3}\right)_{v} \\
& T_{6}^{\mu}=\left(p_{1}^{2}-p_{2}^{2}\right) \gamma^{\mu}+\left(p_{1}+p_{2}\right)^{\mu} \not p_{3} \\
& T_{7}^{\mu}=\frac{\mathrm{i}}{2}\left(p_{1}^{2}-p_{2}^{2}\right)\left[\left(\not p_{1}+\not p_{2}\right) \gamma^{\mu}-\left(p_{1}+p_{2}\right)^{\mu}\right]-\mathrm{i}\left(p_{1}+p_{2}\right)^{\mu} \sigma_{\lambda v} p_{2}^{\lambda} p_{1}^{v} \\
& T_{8}^{\mu}=-\gamma^{\mu} \sigma_{\lambda v} p_{2}^{\lambda} p_{1}^{v}-\not p_{2} p_{1}^{\mu}+\not p_{1} p_{2}^{\mu}
\end{aligned}
$$

with $p_{3}=p_{2}-p_{1}$ and $\sigma_{\mu \nu}=\frac{1}{2}\left(\gamma_{\mu} \gamma_{\nu}-\gamma_{\nu} \gamma_{\mu}\right)$. This basis is similar to that of [11], differing only in the momentum labels. The structures $L_{1}, L_{2}, T_{2}, T_{3}$ and $T_{6}$ each involve one Dirac matrix and therefore form the Dirac-vector part of the vertex. The structures $L_{3}$ and $T_{1}$ form the scalar part of the vertex, whereas the remaining structures $L_{4}, T_{4}, T_{5}$ and $T_{8}$ have tensorial character. For the purpose of our powercounting analysis as given in Section 3.4 the tensors $L_{4}, T_{4}, T_{5}$ with an even number of Dirac matrices act similar to the scalar ones, whereas the tensor $T_{8}$ with an odd number of Dirac matrices acts similar to the vector part.

\section{A.2. On the kinematics of the quark-gluon vertex}

Since the quark-gluon vertex Dirac amplitudes $\lambda_{i}$ and $\tau_{i}$, defined in Eq. (2), are functions of three Euclidean scalars $p_{q}^{2}, p_{\bar{q}}^{2}, p_{g}^{2}$, it is customary to plot them in slices of this three-dimensional space, that we denote "kinematic sections". Given the various choices in the literature, we find it convenient to collect here definitions for the most used sections.

First, there are important kinematic sections that are "degenerate", in the sense that there is only one independent vector $p_{\mu}$ in four-dimensional Euclidean space instead of the usual two vectors $p_{q}^{\mu}, p_{\bar{q}}^{\mu}$ (the gluon momentum being in the plane spanned by them).

A popular degenerate section is the "soft gluon" section, characterized by a gluon of exactly zero momentum

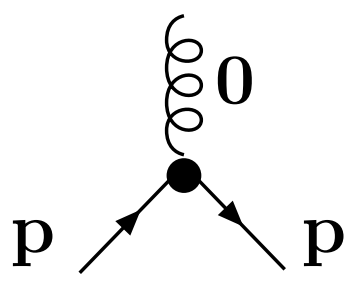

A similar section is the "soft quark" section where one quark momentum vanishes. This corresponds in the chiral limit to an on-shell quark in Minkowski space: 


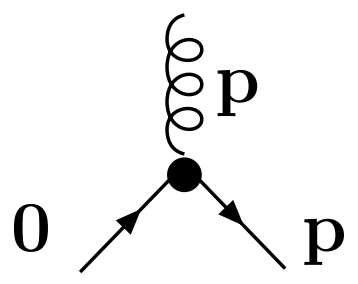

Next let us look at two interesting non-degenerate sections. We introduce the so called "1-2-3" or "totally asymmetric" section by

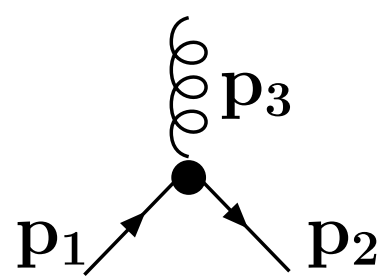

imposing the restrictions (in terms of $p_{1}^{2} \equiv p^{2}$ ), $p_{2}^{2}=2 p^{2}, p_{3}^{2}=3 p^{2}$ that fix the relative angle of $p_{1}, p_{2}$ and the quotient of their Euclidean moduli.

The other section is the so called "symmetric" section given by $p_{1}^{2}=p_{2}^{2}=p_{3}^{2} \equiv p^{2}$, in which all scales flowing into the vertex have equal value (but $p_{1}$ and $p_{2}$ are not parallel).

These four sections are in principle useful to study the infrared behavior of the vertex, that is, the infrared exponents. To expose the angular dependence of the vertex dressing functions, it is convenient to define a "fixed scale" section in which we vary the angle between $p_{1}$ and $p_{2}$ but fix $p_{1}^{2}=p_{2}^{2} \equiv p^{2} \neq 0$. Thus, we can plot $\lambda_{i}(x)$ in terms of the polar cosine

$$
x=\frac{p_{1} \cdot p_{2}}{\left|p_{1}\right|\left|p_{2}\right|}=1-\frac{p_{3}^{2}}{2 p^{2}} \text {. }
$$

\section{Appendix B. Second DSE formulation for the quark-gluon vertex}

In Section 3 in the main part of this work we gave a solution for the infrared behavior of the quarkgluon vertex in terms of power laws. There it turned out that the vertex has both on overall infrared singularity of degree $\left(p^{2}\right)^{-1 / 2-\kappa}$ when all external momenta are proportional to one scale $p$ which goes to zero and also a collinear singularity $\left(p_{g}^{2}\right)^{-1 / 2-\kappa}$ when only the gluon momentum $p_{g}$ is small compared to $\Lambda_{\mathrm{QCD}}$.

This solution has been derived from a version of the vertex-DSE, where the bare vertex is always the one attached to the external gluon leg. This gives the equation shown in Fig. 3 in the main body of this work, which includes all possible bare vertices from the Lagrangian of the theory. We will call this version of the DSE 's-channel version' in the following. This solution also straightforwardly solves the corresponding equation of motion for the vertex from an 3PI effective action, given in Fig. 5. However, things are slightly more complicated for the second or " $t$-channel" version of the vertex-DSE. This version has a bare vertex attached to an external quark line and is given in Fig. 4.

The problem here is that the simple triangle diagrams involving only two- and three-point functions do not reproduce the infrared singularity found from the other two equations. Let us carry through this exercise for example for the diagram involving the fully dressed three-gluon vertex. Simple power counting then results in an overall divergence of 


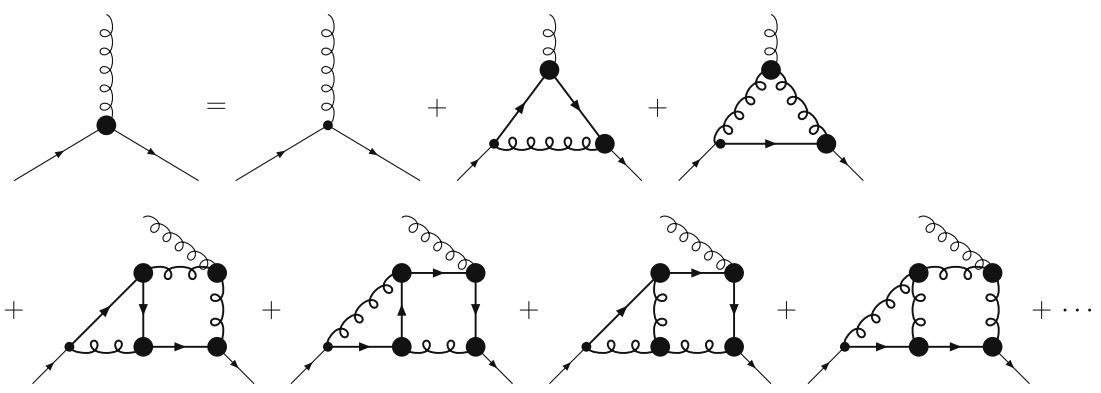

Fig. 24. Leading contributions in the skeleton expansion of the t-channel-version of the DSE for the quark-gluon vertex.

$$
\left(p^{2}\right)^{-3 \kappa+1 / 2} \times\left(p^{2}\right)^{2 \kappa-1} \times\left(p^{2}\right)^{2 \kappa-1} \times\left(p^{2}\right)^{-1 / 2-\kappa} \times\left(p^{2}\right)^{2} \sim\left(p^{2}\right)^{0},
$$

where the first term stems from the three-gluon vertex, the next two terms from the gluon propagators followed by the scalar part of the remaining quark-gluon vertex and the loop integration, whereas the quark propagator is constant. To yield the previous IR divergence of the vector part of the vertex on the left hand side there is clearly infrared strength missing.

The answer to this puzzle lies in the remaining diagram, that includes the 1PI gluon-quark fourpoint function. Since the ghost dynamics is the dominant dynamical contribution in the DSEs and there is no fundamental quark-ghost coupling, similarly to the case of the s-channel DSE discussed in detail in the main text, it is again necessary to perform a skeleton expansion to two-loop order as shown in Fig. 24.

The power-counting analysis for this equation is complicated since not only soft modes of the order of the external momenta can dominate the different loop integrals but also hard modes of the order of the dynamically-generated quark mass $M$ appearing in the quark propagators. ${ }^{13}$ In combination with the different possibilities to route any hard external momenta through the diagrams, the two-loop integrals can be decomposed into many different potentially IR sensitive regions [23]. The most singular contribution on the right hand side of the DSE dominates and will determine the scaling of the vertex on the left hand side. Naturally, Greens functions involving only hard momenta which appear in a given graph do not scale with the soft external momentum. Here, we will not give a complete power-counting analysis analogous to the one for the first DSE presented in Appendix E, but rather identify the contributions that provide the strong singularities found in the s-channel DSE discussed in the main text.

Let us start with the quark-gluon vertex in the soft-gluon limit. One particular contribution arising from a specific kinematic region of the first two-loop graph in Fig. 24 is given in Fig. 25(a). Here, the loop integral of the initial graph (left part) arising in the unexpanded DSE Fig. 4 extends over hard momenta whereas only the loop integral (right part) arising from the skeleton expansion involves soft scales of the order of the external gluon momentum. ${ }^{14}$ In the case that this contribution dominates it yields for the anomalous exponent of the quark-gluon vertex in the soft-gluon limit the self-consistent result (counting only IR exponents)

$$
\delta_{q g}^{g l}=2+\left(\frac{1}{2}+\delta_{3 g}^{u}\right)+2 \delta_{q g}^{g l}+2\left(\delta_{g l}-1\right)=2 \delta_{q g}^{g l}+\frac{1}{2}+\kappa \Rightarrow \delta_{q g}^{g l}=-\frac{1}{2}-\kappa
$$

Accordingly the ghost-enhanced three-gluon vertex induces a strong soft-gluon singularity in full analogy to the s-channel version discussed in the main text. However, this mechanism does not generate a uniform divergence in the absence of soft divergences. To see how the latter is generated selfconsistently consider graph (b) of Fig. 25 which presents one of the possible contributions. If it dominates it yields for the corresponding anomalous exponent

\footnotetext{
${ }^{13}$ We note that a very similar integral appears in the DSE for the quark propagator, which is very well explored. These integrals are known to result in finite answers in the infrared for a wide range of possible dressings in the loops (see e.g., [9]).

${ }^{14}$ Note that we are only interested in the scaling with respect to the soft external momentum $p \ll \Lambda_{\text {QCD }}$ and do not distinguish between any hard external momenta $q<\Lambda_{\mathrm{QCD}}$ and hard loop momenta $q \sim M$.
} 

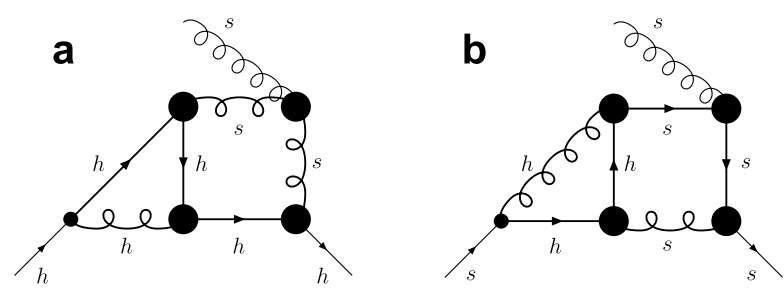

Fig. 25. Kinematic configurations of the leading loop graphs that generate the strong soft-gluon divergence (a) and the uniform divergence (b) of the quark-gluon vertex. The labels $s$ and $h$ denote whether the momentum running through a line is soft or hard, respectively. In contrast to the s-channel-version of the DSE, here the soft-gluon singularity in graph (b) is necessary to induce the uniform divergence.

$$
\delta_{q g}^{u}=2+2 \delta_{q g}^{u}+\delta_{q g}^{g l}+\delta_{q g}^{q}+\left(\delta_{g l}-1\right)+2 \delta_{q}=2 \delta_{q g}^{u}+\frac{1}{2}+\kappa \Rightarrow \delta_{q g}^{u}=-\frac{1}{2}-\kappa .
$$

We therefore conclude that the structure of the loop integrals in the last term of Fig. 4 together with the collinear singularity in the external gluon leg provides exactly such strength in the infrared to reproduce our results for the quark-gluon vertex in the s-channel version of the vertex-DSE also for the t-channel version.

It is interesting to note a few properties of this result: first, in contrast to the s-channel DSE, here regions of the loop integral with hard momenta of the order of the quark mass dominate even in the uniform limit. Further, the uniform divergence in the t-channel DSE is not directly induced by the three-gluon vertex but only indirectly once the strong soft-gluon divergence is present. Therefore, in the present case these two divergences are not totally independent but imply each other. Furthermore, in the t-channel version it is not necessary to distinguish between the different Dirac components of the vertex to recover the proper uniform scaling. The reason is that the corresponding graphs involve many fermionic Greens functions that involve hard momenta and do not scale with the soft external momentum, independent of their Dirac-structure. Correspondingly, in contrast to the case of the non-Abelian graph studied in the main text, there are no corresponding restrictions, here.

Finally, from a practical point of view the s-channel version of the DSE lends itself much better to a numerical analysis, since, as explained in the main text, the IR dominant two-loop graph can be approximately replaced by a 1-loop graph with a dressed three-gluon vertex. In the quenched approximation this three-gluon vertex can be computed beforehand or, as done in the present analysis, simply be implemented by some ansatz. In the t-channel version of the DSE this is not possible since the initial loop dresses the desired quark-gluon vertex itself and therefore the expensive two-loop integrals indeed would have to be analyzed numerically.

\section{Appendix C. Semi-perturbative analysis of the quark-gluon vertex}

\section{C.1. Non-Abelian diagram}

We start by evaluating the one-loop diagram involving the non-Abelian three gluon vertex

$$
\operatorname{igT} T_{i j}^{a} \Gamma_{\mathrm{NA}}^{\mu}\left(p 1^{2}, p_{2}^{2},\left(p_{2}-p_{1}\right)^{2}\right)=k_{1}=p_{1}-q
$$


The color structure for this diagram is

$$
\frac{\mathrm{i} N_{c}}{2} T_{i j}^{a}
$$

where $T_{i j}^{a}$ is the generator in the fundamental representation, and the factor $N_{c}$ results from the contraction of the color matrices. The full expression reads

$$
\begin{aligned}
\Gamma_{\mathrm{NA}}^{\mu}\left(p 1^{2}, p_{2}^{2},\left(p_{2}-p_{1}\right)^{2}\right)= & \left(\frac{-\mathrm{i} N_{c}}{2} g^{2} Z_{1 \mathrm{~F}}^{2} Z_{3}\right) \int \frac{\mathrm{d}^{4} q}{(2 \pi)^{4}}\left\{\gamma^{v}\left(\mathrm{i} \not q \sigma_{\mathrm{v}}\left(q^{2}\right)+\sigma_{\mathrm{s}}\left(q^{2}\right)\right) \gamma^{\rho} \frac{Z\left(k_{1}^{2}\right)}{k_{1}^{2}} \frac{Z\left(k_{2}^{2}\right)}{k_{2}^{2}}\right. \\
& \left.\times\left(\delta_{\sigma \theta}\left(k_{1}-k_{2}\right)_{\mu}+\delta_{\theta \mu}\left(k_{2}-k_{3}\right)_{\sigma}+\delta_{\sigma \mu}\left(k_{3}-k_{1}\right)_{\theta}\right)\left(\delta_{v \sigma}-\frac{k_{1 v} k_{1 \sigma}}{k_{1}^{2}}\right)\left(\delta_{\rho \theta}-\frac{k_{2 \rho} k_{2 \theta}}{k_{2}^{2}}\right)\right\},
\end{aligned}
$$

with the renormalized coupling $g^{2}$ and the renormalization constants $Z_{1 F}$ and $Z_{3}$ for the bare quarkgluon vertices and the three-gluon vertex. Dimensional analysis of Eq. (C2) immediately shows a logarithmic divergence which appears only in $\lambda_{1}$. All other pieces of the vertex, containing powers of either $p_{i}$, are free of ultraviolet divergences. Our renormalization prescription for $\lambda_{1}$ is given in Section 4.1.

\section{C.2. Abelian vertex diagram}

Next we turn our attention to the "QED-like" diagram

$$
\operatorname{ig} T_{i j}^{a} \Gamma_{\mathrm{A}}^{\mu}\left(p 1^{2}, p_{2}^{2},\left(p_{2}-p_{1}\right)^{2}\right)=k_{1}=p_{1}-q
$$

This has color structure

$$
\frac{-1}{2 N_{c}} T_{i j}^{a}
$$

and reads

$$
\begin{aligned}
\Gamma_{A}^{\mu}\left(p_{1}^{2}, p_{2}^{2},\left(p_{2}-p_{1}\right)^{2}\right)= & \left(\frac{1}{2 N_{c}} g^{2}\right) \int \frac{\mathrm{d}^{4} q}{(2 \pi)^{4}}\left\{\frac{Z\left(q^{2}\right)}{q^{2}} \gamma^{v}\left(\mathrm{i} k_{1} \sigma_{\mathrm{v}}\left(k_{1}^{2}\right)+\sigma_{\mathrm{s}}\left(k_{1}^{2}\right)\right) \gamma^{\mu}\left(-\mathrm{i} k_{2} \sigma_{\mathrm{v}}\left(k_{2}^{2}\right)+\sigma_{\mathrm{s}}\left(k_{2}^{2}\right)\right) \gamma^{\rho}\right. \\
& \left.\times\left(\delta_{v \rho}-\frac{q_{v} q_{\rho}}{q^{2}}\right)\right\} .
\end{aligned}
$$

Both the Abelian and the non-Abelian diagrams reproduce important properties of the vertex; as discussed in [10], charge conjugation invariance requires

$$
C \gamma^{\mu} C^{-1}=-\gamma^{\mu \mathrm{T}}, \quad C \mathrm{i} \Gamma^{\mu} C^{-1}=\mathrm{i} \Gamma^{\mu \mathrm{T}},
$$

where $C$ is the charge conjugation operator and the superscript "T" denotes transposition. Referring to the Ball-Chiu basis (A2) above, we see that the $\lambda_{4}, \tau_{4}$, and $\tau_{6}$ Dirac amplitudes should be odd under the interchange of $p_{1}$ and $p_{2}$, whereas the other nine should be even. This is indeed the case for both diagrams in our calculation. Also, the anti-unitarity of the time reversal operator means that all the Dirac amplitudes should be either real or imaginary. With the explicit factors of $i$ in Eq. (1) and the conventions of (A2) all the $\lambda$ and $\tau$ are strictly real. 


\section{C.3. Input for gluon and quark propagators}

The dressing functions for the gluon $Z$ and quark $\sigma_{\mathrm{v}}, \sigma_{\mathrm{s}}$ propagators are taken as input from reference [110]. We employ analytical fits to the numerical solution of the Schwinger-Dyson equations from this work corresponding to a choice $\alpha_{s}=\frac{g^{2}}{4 \pi}=0.2$. The gluon propagator is well represented by

$$
Z(x)=\left(\frac{\alpha(x)}{\alpha(\mu)}\right)^{1+2 \delta} R^{2}(x)
$$

and the ghost propagator by

$$
G(x)=\left(\frac{\alpha(x)}{\alpha(\mu)}\right)^{-\delta} R^{-1}(x),
$$

where the low momentum behavior is captured by the irrational function

$$
R(x)=\frac{c\left(\frac{x}{\Lambda^{2}}\right)^{\kappa}+d\left(\frac{x}{\Lambda^{2}}\right)^{2 \kappa}}{1+c\left(\frac{x}{\Lambda^{2}}\right)^{\kappa}+d\left(\frac{x}{\Lambda^{2}}\right)^{2 \kappa}} .
$$

The parameters appearing in $R(x)$, solution of the DSE are $c=1.269$ and $d=2.105$, and in the quenched approximation for the gluon and ghost equations $\delta\left(N_{f}=0\right)=-9 / 44$. The value for the exponent $\kappa \simeq 0.595$ in this fit is approximately known from the infrared analysis of the DSEs [7].

Finally, the running coupling is well reproduced by

$$
\alpha(x)=\frac{\alpha(0)}{\log \left(e^{1}+a_{1}\left(\frac{x}{\Lambda^{2}}\right)^{a_{2}}+b_{1}\left(\frac{x}{\Lambda^{2}}\right)^{b_{2}}\right)}
$$

with $a_{1}=1.106, a_{2}=2.324, b_{1}=0.004, b_{2}=3.169$. Using a $\widetilde{M O M}$ scheme and fitting $\Lambda$ to only the ultraviolet behavior, a value $\Lambda_{\mathrm{QCD}}=0.714 \mathrm{GeV}$ has been given in Ref. [9]. Note, that these fits are for faster computing and to offer a closed form: all results for the various two-point functions are solutions of their respective Schwinger-Dyson equations. These fits will be used for all numerical calculations presented in the course of this work.

In the same way we can parameterize the quark propagator functions, $\sigma_{\mathrm{s}, \mathrm{v}}\left(p^{2}\right)$, and fit these to the numerical DSE solutions. Again, we want to make explicit the leading logarithmic corrections to $Z^{f}\left(p^{2}\right)$ and $M\left(p^{2}\right)$, achieved with

$$
\begin{aligned}
\sigma_{\mathrm{s}}\left(p^{2}\right) & =\frac{B_{\text {chiral }}\left(p^{2}\right)}{p^{2}+m_{\text {sing }}^{2}}+\frac{C_{\text {cqm }} \alpha\left(p^{2}+m_{\text {sing }}^{2}\right)^{\gamma_{m}}}{p^{2}+m_{\text {sing }}^{2}}, \\
\sigma_{\mathrm{v}}\left(p^{2}\right) & =\frac{1}{p^{2}+m_{\text {sing }}^{2}}\left(1-\frac{\alpha\left(p^{2}+m_{\text {sing }}^{2}\right)}{2 \pi}+\widetilde{C} B_{\text {chiral }}\left(p^{2}\right)\right),
\end{aligned}
$$

with

$$
B_{\text {chiral }}\left(p^{2}\right)=C_{d c s b} \frac{\alpha\left(p^{2}+m_{\text {sing }}^{2}\right)^{1-\gamma_{m}}}{p^{2}+m_{\text {sing }}^{2}+\Lambda^{2}}+\frac{C_{4}}{\left(p^{2}+m_{\text {sing }}^{2}+\Lambda^{2}\right)^{2}} .
$$

This parameterization ensures the correct asymptotic behavior for $\sigma_{s, \mathrm{~V}}\left(p^{2}\right)$ and the quark functions $M\left(p^{2}\right)$ and $Z^{f}\left(p^{2}\right)[9,53]$ in the chiral limit. It is important to note that we use this fit for the quark propagator in this appendix only! In the main text, where we back-feed the vertex into the quark-DSE, given in Fig. 1, the corresponding quark propagator is solved self-consistently and dynamically from the set of coupled DSEs for the quark propagator and the quark-gluon vertex. With this input for the propagators the loop corrections Eq. (C1) and (C3) are straightforwardly evaluated and yield the result shown in Fig. 7 in the main text. 


\section{Appendix D. Power-counting and Dirac structure: an explicit example}

In this appendix we demonstrate the fact that the power-counting analysis depends only on the Diracscalar or vector nature of the individual tensors of the quark-gluon vertex explicitly in a special kinematic limit. Due to momentum conservation the vertex depends only on the two external quark momenta $\left(p_{1}\right)_{\mu}$, $\left(p_{2}\right)_{\mu}$ or three Lorentz invariants $p_{1}^{2}, p_{2}^{2}, p_{1} \cdot p_{2}$. To analyze the infrared limit of this vertex in the presence of only one external scale $p_{1}^{2} \equiv p^{2} \ll \Lambda_{\mathrm{QCD}}^{2}$, we can set $\left(p_{2}\right)_{\mu}=2\left(p_{1}\right)_{\mu}=: 2 p_{\mu}$ without loss of generality. (Note that the case $\left(p_{2}\right)_{\mu}=\left(p_{1}\right)_{\mu}$ corresponds to the collinear limit where the gluon momentum goes to zero, while the other momenta stay finite. We treat this limit separately in Section 3.5.)

The choice $\left(p_{2}\right)_{\mu}=2\left(p_{1}\right)_{\mu}$ leaves us with only four possible tensor structures which can be denoted by

$$
\Gamma_{\mu}(p)=\mathrm{ig} \sum_{i=1}^{4} \xi_{i}\left(p^{2}\right) G_{\mu}^{i}
$$

with $\xi_{i}$ being Lorentz and Dirac scalar functions and

$$
G_{\mu}^{1}=\gamma_{\mu}, G_{\mu}^{2}=\hat{p}_{\mu}, G_{\mu}^{3}=\hat{\not p} \hat{p}_{\mu}, G_{\mu}^{4}=\hat{\not p} \gamma_{\mu},
$$

where we have normalized the momentum, $\hat{p}_{\mu}=p_{\mu} / \sqrt{p^{2}}$, to ease power-counting. Note that $G_{\mu}^{1}$ and $G_{\mu}^{3}$ have an odd number of $\gamma$-matrices, whereas $G_{\mu}^{2}$ and $G_{\mu}^{4}$ have an even number. Therefore, $\xi_{2,4} \neq 0$ only if chiral symmetry is broken.

To project out the respective dressing functions $\xi_{1 \ldots 4}\left(p^{2}\right)$ from the vertex-DSE we multiply the equation with $\gamma_{\mu}, \hat{p}_{\mu}, \hat{p p} \hat{p}_{\mu}, \hat{p} \gamma_{\mu}$, respectively, and take the Dirac-trace. Linear combinations of these four basis elements then give the desired $\xi_{1 \ldots 4}\left(p^{2}\right)$.

For the internal quark-gluon vertices we can employ the expression (D1) with any internal momentum as argument: it contains all possible types of Dirac structures (vector, scalar and tensor). Any more complicated dependence on external and internal momenta will generate the same powers of external momenta in $\xi_{1 . .4}$ after integration (for dimensional reasons). To determine the infrared exponents of the quark-gluon vertex we employ the ansatz

$$
\xi_{1}\left(p^{2}\right)=\chi_{1} \cdot\left(p^{2}\right)^{\beta_{1}}, \quad \xi_{2}\left(p^{2}\right)=\chi_{2} \cdot\left(p^{2}\right)^{\beta_{2}}, \quad \xi_{3}\left(p^{2}\right)=\chi_{3} \cdot\left(p^{2}\right)^{\beta_{3}}, \quad \xi_{4}\left(p^{2}\right)=\chi_{4} \cdot\left(p^{2}\right)^{\beta_{4}}
$$

for the scaling of the dressing functions of the quark-gluon vertex with the momentum scale, where the pre-factors $\chi_{i}$ depend only on momentum ratios.

Since we contracted the right hand side of the DSE with appropriate tensor structures, see above, we eventually have to deal with scalar integrals only, which depend on powers of internal and external momenta. The evaluation of such diagrams has been described in [56]. Most important for our purpose is the fact that all powers of internal momenta transform to powers of external momenta after integration. This is also clear from simple dimensional consideration, provided there is only one external scale present. In our case this is ensured by the condition $p^{2} \ll \Lambda_{\text {QCD }}^{2} \leqslant M^{2}$, which leaves only one scale $p^{2}$ in the deep infrared. The following analysis rests on the assumption that there is no second small scale present. In principle this assumption may not be true for heavy quarks, since then one has a potential additional small scale $\Lambda_{\text {QCD }} / M^{2}$. We have investigated this possibility in our numerical calculations of the quark-gluon vertex and did NOT see any influence of such a quantity. The corresponding results of our quark mass study are presented in Section 4.3, where we discuss this issue further.

A similar analysis can be done using all four dressing functions $\xi_{1 \ldots 4}$. The explicit integral equations are quite lengthy and add nothing to the power-counting argument, so we omit them here. One then obtains:

$$
\begin{aligned}
\beta_{1}= & 1 / 2+\kappa+\min \left\{1 / 2+2 \beta_{1}, 1 / 2+2 \beta_{2}, 1 / 2+2 \beta_{3}, 1 / 2+2 \beta_{4}, 1 / 2+\beta_{1}+\beta_{3}, 1 / 2+\beta_{2}+\beta_{4}, \beta_{1}\right. \\
& \left.+\beta_{2}, \beta_{1}+\beta_{4}, \beta_{2}+\beta_{3}, \beta_{3}+\beta_{4}\right\} \\
\beta_{2}= & 1 / 2+\kappa+\min \left\{1 / 2+\beta_{1}+\beta_{2}, 1 / 2+\beta_{1}+\beta_{4}, 1 / 2+\beta_{2}+\beta_{3}, 1 / 2+\beta_{3}\right. \\
& \left.+\beta_{4}, 2 \beta_{1}, 2 \beta_{2}, 2 \beta_{3}, 2 \beta_{4}, \beta_{1}+\beta_{3}, \beta_{2}+\beta_{4}\right\} \\
\beta_{3}= & 1 / 2+\kappa+\min \left\{1 / 2+2 \beta_{1}, 1 / 2+2 \beta_{2}, 1 / 2+2 \beta_{3}, 1 / 2+2 \beta_{4}, 1 / 2+\beta_{1}+\beta_{3}, 1 / 2+\beta_{2}+\beta_{4}, \beta_{1}\right. \\
& \left.+\beta_{2}, \beta_{1}+\beta_{4}, \beta_{2}+\beta_{3}, \beta_{3}+\beta_{4}\right\}
\end{aligned}
$$




$$
\begin{aligned}
\beta_{4}= & 1 / 2+\kappa+\min \left\{1 / 2+\beta_{1}+\beta_{2}, 1 / 2+\beta_{1}+\beta_{4}, 1 / 2+\beta_{2}+\beta_{3}, 1 / 2+\beta_{3}+\beta_{4}, 2 \beta_{1}, 2 \beta_{3}, 2 \beta_{4}, \beta_{1}\right. \\
& \left.+\beta_{3}, \beta_{2}+\beta_{4}\right\}
\end{aligned}
$$

which is solved by

$$
\begin{aligned}
& \beta_{2}=-1 / 2-\kappa, \quad \beta_{1}, \beta_{3} \in[-1 / 2-\kappa,-\kappa], \\
& \beta_{4} \in[-1 / 2-\kappa, 1 / 2-\kappa] .
\end{aligned}
$$

This leaves a range of possibilities with the common trait that the most infrared singular piece diverges as $\left(p^{2}\right)^{-1 / 2-\kappa}$ in the infrared. We have analyzed this analytic solution in numerical calculations, presented in Section 4, and found that only the case $\beta_{2}=\beta_{1}=\beta_{3}=\beta_{4}=-1 / 2-\kappa$ is realized.

\section{Appendix E. Power counting with kinematic singularities}

In this appendix we present the full power-counting analysis for the quark sector of quenched QCD, given by the coupled system of the quark propagator and the quark-gluon vertex DSE. In particular we take into account the possibility of kinematic singularities of the vertex and do not make assumptions on the IR limit of the quark propagator. The analysis of kinematic singularities follows the corresponding analysis for the gauge sector of the theory presented in [23].

Whereas the propagator is generally given by a single IR exponent $\delta_{q}$, the quark-gluon vertex has in addition to the uniform limit that all momenta vanish $\delta_{q g}^{u}$ also the possibility that only the gluon $\delta_{q g}^{g l}$ or only the quark leg vanishes $\delta_{q g}^{q}$. As noted in Section 3.4 in the main text it is crucial to distinguish between the scalar and vector components in the analysis. We distinguish them by additional lower case letters $v$ for the vector and $s$ for the scalar part, so that the full set of possibly distinct IR exponents is given by $\delta_{q v}, \delta_{q s}, \delta_{q g v}^{u}, \delta_{q g s}^{u}, \delta_{q g v}^{g l}, \delta_{q g s}^{g l}, \delta_{q g v}^{q}$ and $\delta_{q g s}^{q}$. Where the exponents without a tensor specification are used, they stand for both Dirac structures so that terms with all possible combinations arise after decomposition. Finally, we define the canonical exponent of the quark propagator $\chi_{q}$ which is given by $\chi_{q s}=0$ and $\chi_{q v}=1 / 2$ for the two cases, respectively.

The distinction of the tensor components is already important for the quark propagator, where the different components feature a different scaling already at the tree level. In order to perform the power-counting analysis for the DSE of the individual components of the quark propagator we first have to consider the scaling behavior of the appearing inverse of the dressed propagator which is given for the parameterization Eq. (3) by

$$
\begin{aligned}
S^{-1}(p) & =\left(\mathrm{i} \sigma_{\mathrm{v}} \not p+\sigma_{\mathrm{s}}\right)^{-1}=\mathrm{i} \frac{\sigma_{\mathrm{v}}}{\sigma_{\mathrm{v}}^{2} p^{2}+\sigma_{\mathrm{s}}^{2}} \not p-\frac{\sigma_{\mathrm{s}}}{\sigma_{\mathrm{v}}^{2} p^{2}+\sigma_{\mathrm{s}}^{2}} \\
& \rightarrow \mathrm{i}\left(p^{2}\right)^{\delta_{q v}-\min \left(2 \delta_{q v}+1,2 \delta_{q s}\right)} \not p-\left(p^{2}\right)^{\delta_{q s}-\min \left(2 \delta_{q v}+1,2 \delta_{q s}\right)} .
\end{aligned}
$$

The DSE for the vector part of the quark propagator is given in Fig. 26, where the labels $V$ and $S$ next to the propagator and vertices denote vector and scalar components, respectively. This DSE leads to an equation for the corresponding IR exponent

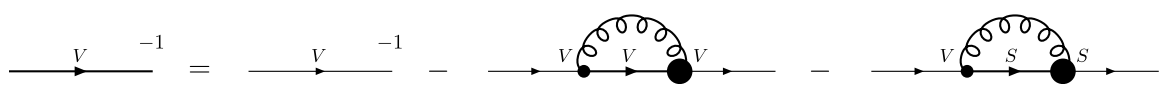

Fig. 26. Decomposed DSE for the vector part of the quark propagator. Vector and scalar components are denoted by $V$ and $S$, respectively.

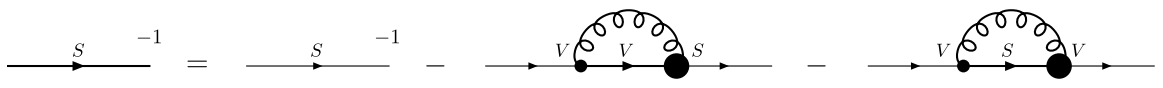

Fig. 27. Decomposed DSE for the scalar part of the quark propagator. 


$$
\delta_{q v}+\frac{1}{2}-\min \left(2 \delta_{q v}+1,2 \delta_{q s}\right)=\min \left(\frac{1}{2}, \delta_{q g v}^{u}+\delta_{q v}+\frac{3}{2}+2 \kappa, \delta_{q g s}^{u}+\delta_{q s}+1+2 \kappa, \delta_{q g v}^{q}+\frac{1}{2}, \delta_{q g s}^{q}+\frac{1}{2}\right) .
$$

Here it has been taken into account that due to a possible quark mass the loop integral can in addition to the IR regime also be dominated by hard modes reflected by the last two terms, where the $\delta_{q g}^{q}$ arises due to the possibility of a soft quark divergence of the vertex and the $1 / 2$ due to a further suppression arising from cancellations owing to gluon transversality. The corresponding DSE for the scalar part of the propagator is shown in Fig. 27 which leads to

$$
\delta_{q s}-\min \left(2 \delta_{q v}+1,2 \delta_{q s}\right)=\min \left(0, \delta_{q g s}^{u}+\delta_{q v}+\frac{3}{2}+2 \kappa, \delta_{q g v}^{u}+\delta_{q s}+1+2 \kappa, \delta_{q g s}^{q}, \delta_{q g v}^{q}\right) .
$$

The DSE for the quark-gluon vertex in a skeleton expansion was given in Fig. 8. When hard scales are present that do not scale to zero there are different regions of the loop integration that can be IR sensitive. In the uniform case there are two possibilities corresponding to a soft or hard loop momentum, respectively. In the soft-particle limits different propagators in the graphs can involve both soft and hard momenta and there is one additional IR sensitive region of the loop integral given by a narrow window of hard modes. This corresponds to a second, not equivalent way to route the large momentum through the loop. When assessing the counting of the vertex, each of these regions could dominate and determine the scaling of the corresponding Greens function on the left hand side. Employing the IR exponents from the Yang-Mills sector in Table 1 yields the equations for the IR-exponents of the quark-gluon vertex in the different limits

$$
\begin{aligned}
\delta_{q g}^{u}= & \min \left(0 ; 2 \delta_{q g}^{u}+\delta_{q}+\chi_{q}+\frac{1}{2}+\kappa, 2 \delta_{q g}^{q}+1-2 \kappa ; 2 \delta_{q g}^{u}+\delta_{q}+\chi_{q}+\frac{1}{2}+4 \kappa, 2 \delta_{q g}^{q} ; 2 \delta_{q g}^{u}+2 \delta_{q}+2 \chi_{q}\right. \\
& \left.+1+2 \kappa, 2 \delta_{q g}^{q}\right) \\
\delta_{q g}^{g l}= & \min \left(0 ; 2 \delta_{q g}^{g l}+\frac{1}{2}+\kappa, 2 \delta_{q g}^{q}+\delta_{q}+\chi_{q}+3-2 \kappa, 1-2 \kappa ; 2 \delta_{q g}^{g l}+\frac{1}{2}+4 \kappa\right. \\
& \left.2 \delta_{q g}^{q}+\delta_{q}+\chi_{q}+2,0 ; 2 \delta_{q g}^{g l}+1+2 \kappa, 2 \delta_{q g}^{q}+2 \delta_{q}+2 \chi_{q}+2,0\right) \\
\delta_{q g}^{q}= & \min \left(0 ; \delta_{q g}^{u}+\delta_{q g}^{q}+\delta_{q}+\chi_{q}+2, \delta_{q g}^{g l}+\delta_{q g}^{q}+2, \delta_{q g}^{q} ; \delta_{q g}^{u}+\delta_{q g}^{q}+\delta_{q}+\chi_{q}+1+2 \kappa\right. \\
& \left.\delta_{q g}^{g l}+\delta_{q g}^{q}+1+2 \kappa, \delta_{q g}^{q} ; 2 \delta_{q g}^{q}+\delta_{q}+\chi_{q}+2, \delta_{q g}^{u}+\delta_{q g}^{g l}+\delta_{q}+\chi_{q}+1+2 \kappa, \delta_{q g}^{q}\right)
\end{aligned}
$$

where semicolons separate the contributions from the first four graphs in Fig. 8 and commas separate those from different regions of the same loop integration. Since the ghost-loop contribution in the first diagram in Fig. 8 is IR divergent it is immediately clear, and reflected in the power-counting above, that the second (non-Abelian) graph is subleading.

Although the remaining system of equations for the quark sector looks extremely involved, it is largely simplified by powerful constraints arising from it. In particular, the nonlinear terms on the right hand sides of the vertex equations yield the constraints

$$
\delta_{q g}^{u}+\delta_{q}+\chi_{q}+\frac{1}{2}+\kappa \geqslant 0, \quad \delta_{q g}^{g l}+\frac{1}{2}+\kappa \geqslant 0, \quad \delta_{q g}^{q}+\delta_{q}+\chi_{q}+2 \geqslant 0 .
$$

Using these constraints in the above equations obtained from the DSEs as well as the known possible range for $\kappa$ [23] they precisely cancel the nonlinear contributions from the Abelian graph in the uniform and the soft-gluon limit as well as all non-trivial contributions in the soft-quark limit and the propagator equations. Dropping the distinction between contributions from the different graphs, the remaining system reads

$$
\begin{aligned}
& \delta_{q v}-\min \left(2 \delta_{q v}+1,2 \delta_{q s}\right)=\min \left(0, \delta_{q g v}^{q}, \delta_{q g s}^{q}\right), \\
& \delta_{q s}-\min \left(2 \delta_{q v}+1,2 \delta_{q s}\right)=\min \left(0, \delta_{q g s}^{q}, \delta_{q g v}^{q}\right), \\
& \delta_{q g}^{u}=\min \left(0,2 \delta_{q g}^{u}+\delta_{q}+\chi_{q}+\frac{1}{2}+\kappa, 2 \delta_{q g}^{q}+1-2 \kappa\right),
\end{aligned}
$$




$$
\begin{aligned}
& \delta_{q g}^{g l}=\min \left(2 \delta_{q g}^{g l}+\frac{1}{2}+\kappa, 2 \delta_{q g}^{q}+\delta_{q}+\chi_{q}+3-2 \kappa, 2 \delta_{q g}^{q}+2 \delta_{q}+2 \chi_{q}+2,1-2 \kappa\right), \\
& \delta_{q g}^{q}=\min \left(0, \delta_{q g}^{q}\right) .
\end{aligned}
$$

To solve the system let us start with the equation for the soft-quark limit of the quark-gluon vertex. From a purely mathematical point of view, the second element in the minimum function yields a condition which is trivially fulfilled for any $\delta_{q g}^{q}<0$ that is compatible with all constraints from the other equations. However, since the perturbative vertex does not contain a soft-quark divergence and there is no dynamical contribution that could induce a singularity we conclude that $\delta_{q g}^{q}=0$ is the only possible physical solution.

Next consider the equations for the propagators. Due to the trivial vertex in the soft-quark limit they reduce to

$$
\delta_{q v}=\min \left(2 \delta_{q v}+1,2 \delta_{q s}\right), \quad \delta_{q s}=\min \left(2 \delta_{q v}+1,2 \delta_{q s}\right) .
$$

It is easy to see that this system only has the trivial solution $\delta_{q}=\delta_{q v}=\delta_{q s}=0$. Correspondingly the self-consistent solution has the same structure as the bare propagator with a mass term that suppresses IR propagation. This mass is naturally expected to be enhanced by spontaneous symmetry breaking.

As can be seen from Eqs. (E10) and (E11) the vertex equation in the soft-gluon limit is totally decoupled from the equation in the uniform limit. Therefore, it is possible to solve the soft-gluon equation first which is considerably simplified by the trivial solution for the propagators and the vertex in the soft-quark limit. Due to the absence of propagators in the equation there are no restrictions from the Dirac structure in this case. Thereby the exponents for the scalar and vector part are equal and it is not necessary to decompose the equation into different tensor components. The remaining equation reads

$$
\delta_{q g}^{g l}=\min \left(2 \delta_{q g}^{g l}+\frac{1}{2}+\kappa, 1-2 \kappa\right)
$$

and has the two solutions

$$
\delta_{q g}^{g l}=-\frac{1}{2}-\kappa \quad \vee \quad \delta_{q g}^{g l}=1-2 \kappa .
$$

The first one is a strong IR divergence that is self-consistently enhanced by the quark dynamics and which precisely saturates the above bound, whereas the second one is the mild singularity fed in from the corresponding soft singularities in the gauge sector.

As has been noted before in the main text, due to the different scaling of the two components of the propagators, the remaining uniform vertex equation has to be decomposed into two equations for scalar and vector components. This decomposition is performed for the remaining IR leading diagram in Figs. 28 and 29.

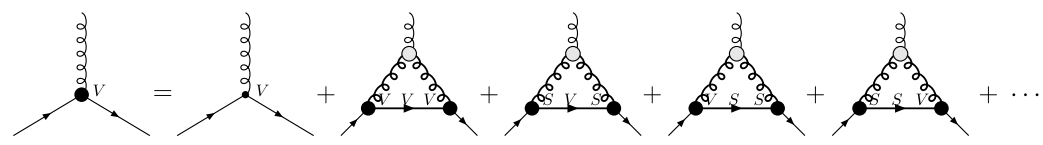

Fig. 28. The vector part in the decomposition of the IR-leading order of the skeleton expansion of the quark-gluon vertex. The open blob represents the ghost triangle in Fig. 8 that represents the IR-leading contribution to the 3-gluon vertex.

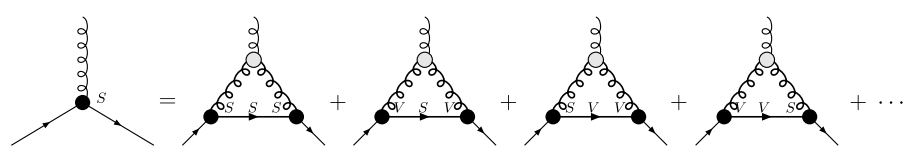

Fig. 29. The scalar part in the decomposition of the IR-leading order of the skeleton expansion of the quark-gluon vertex. 
Table 7

The IR-exponents for the leading Green's functions of the possible IR fixed points of the quark sector of quenched QCD

\begin{tabular}{llll}
\hline$\delta_{q}$ & $\delta_{q g}^{u}$ & $\delta_{q g}^{q}$ & $\delta_{q g}^{g l}$ \\
\hline 0 & $-\frac{1}{2}-\kappa \vee 1-2 \kappa$ & 0 & $-\frac{1}{2}-\kappa \vee 1-2 \kappa$ \\
\hline
\end{tabular}

The scalar Dirac parts are of leading order in the IR.

Correspondingly, the remaining system for the components of the vertex in the uniform limit is given by

$$
\begin{aligned}
& \delta_{q g v}^{u}=\min \left(2 \delta_{q g v}^{u}+\delta_{q v}+1+\kappa, 2 \delta_{q g s}^{u}+\delta_{q v}+1+\kappa, \delta_{q g v}^{u}+\delta_{q g s}^{u}+\delta_{q s}+\frac{1}{2}+\kappa, 1-2 \kappa\right), \\
& \delta_{q g s}^{u}=\min \left(2 \delta_{q g s}^{u}+\delta_{q s}+\frac{1}{2}+\kappa, 2 \delta_{q g v}^{u}+\delta_{q s}+\frac{1}{2}+\kappa, \delta_{q g v}^{u}+\delta_{q g s}^{u}+\delta_{q v}+1+\kappa, 1-2 \kappa\right) .
\end{aligned}
$$

Aside from the last terms in these equations arising from the mild soft gluon divergence of the 3gluon vertex, this is the system that has been discussed in detail in Section 3.4 and it is not affected by the soft singularities. As in the case of the soft-gluon singularity discussed above, these latter terms in principle also allow for the possibility of a weaker singularity $\delta_{q g}^{u}=-1 / 2-\kappa$ that is not self-consistently enhanced but only induced from the gauge sector. However, our numerical results presented in Section 4 clearly favor the strong divergences as a solution of the full DSE system.

So far we have distinguished between the scalar and vector components of quark correlation functions since this was crucial to obtain the correct IR exponents that properly take into account the Dirac structure. This distinction is surely also important to assess the chiral properties of the above solution and we recall once more that the scalar part is crucial. Aside from this, however, now that the solutions have been found these are just different parts of the same Green's function. Thus, analogously to the power-counting in the gauge sector where only the IR exponents of the leading tensor structure(s) are considered, the solutions for the IR-leading behavior of the quark sector of quenched QCD is given in Table 7.

\section{Appendix F. Dirac algebra}

Skipping for now the color index to concentrate on the Dirac space indices, the vertex in Eq. (C2) can be decomposed with the help of Lorentz invariance and the sign convention in Eq. (A2) as

$$
\begin{aligned}
\Gamma_{\mathrm{NA}}^{\mu}= & \Gamma_{1} p_{1}^{\mu}+\Gamma_{2} p_{2}^{\mu}+\Gamma_{3} \gamma^{\mu}+\Gamma_{4} \not p_{1} p_{1}^{\mu}+\Gamma_{5} \not p_{1} p_{2}^{\mu}+\Gamma_{6} \not p_{2} p_{1}^{\mu}+\Gamma_{7} \not p_{2} p_{2}^{\mu}+\Gamma_{8} \not p_{1} \gamma^{\mu}+\Gamma_{9} \not p_{2} \gamma^{\mu} \\
& +\Gamma_{10} \not p_{1} \not p_{2} p_{1}^{\mu}+\Gamma_{11} \not p_{1} \not p_{2} p_{2}^{\mu}+\Gamma_{12} \not p_{1} \not p_{2} \gamma_{\mu} .
\end{aligned}
$$

Our computer codes will calculate the vertex as a Dirac matrix each of whose elements is in turn an integral over momentum space. Once this matrix is obtained we need to project out the various structures in Eq. (F1). Now we briefly describe the algorithm that achieves this. First choose (without loss of generality) a coordinate frame in which $p_{1}=\left(p_{1}, 0,0,0\right)$ and $p_{2}=\left(p_{2} \cos \chi, 0,0, p_{2} \sin \chi\right)$. Then pick a vector $R$ of unit norm $R \cdot R=1$ along the axis $1,2,4$ alternatively and numerically compute $R \cdot \Gamma$. The possible structures appearing in this projected vertex are now:

$$
R \cdot \bar{\Gamma}_{\mathrm{NA}}=\bar{\Gamma}_{1} \mathbf{1}+\bar{\Gamma}_{2} R+\bar{\Gamma}_{3} \not p_{1}+\bar{\Gamma}_{4} \not p_{2}+\bar{\Gamma}_{5} \not p_{1} R+\bar{\Gamma}_{6} \not p_{2} \not R+\bar{\Gamma}_{7} \not p_{1} \not p_{2}+\bar{\Gamma}_{8} \not p_{1} \not p_{2} R,
$$

where $R \cdot \bar{\Gamma}_{\mathrm{NA}}$ takes different values after projection and subtraction in the following three-step algorithm, like in Eqs. (F6) and (F7) below, and the various $\bar{\Gamma}_{i}$ are functions of the (Euclidean) scalars $\left(p 1^{2}, p 2^{2}, p 1 \cdot p 2\right)$.

They can easily be obtained by tracing the vertex $R \cdot \bar{\Gamma}$ with various projectors: 


$$
\begin{aligned}
& {\left[\begin{array}{l}
\bar{\Gamma}_{1} \\
\bar{\Gamma}_{5} \\
\bar{\Gamma}_{6} \\
\bar{\Gamma}_{7}
\end{array}\right]=\frac{1}{4 \Delta}\left[\begin{array}{cccc}
p_{1}^{2} p_{2}^{2} & \left(2 p_{1} \cdot p_{2} R \cdot p_{2}-p_{2}^{2} R \cdot p_{1}\right) & -p_{1}^{2} R \cdot p_{2} & -p_{1} \cdot p_{2} \\
\left(2 p_{1} \cdot p_{2} R \cdot p_{2}-p_{2}^{2} R \cdot p_{1}\right) & p_{2}^{2} & -p_{1} \cdot p_{2} & -R \cdot p_{2} \\
-p_{1}^{2} R \cdot p_{2} & -p_{1} \cdot p_{2} & p_{1}^{2} & R \cdot p_{1} \\
-p_{1} \cdot p_{2} & -R \cdot p_{2} & R \cdot p_{1} & 1
\end{array}\right]\left[\begin{array}{c}
\operatorname{Tr}\left(R \cdot \bar{\Gamma}_{\mathrm{NA}}\right) \\
\operatorname{Tr}\left(R p_{1} R \cdot \bar{\Gamma}_{\mathrm{NA}}\right) \\
\operatorname{Tr}\left(R p_{2} R \cdot \bar{\Gamma}_{\mathrm{NA}}\right) \\
\operatorname{Tr}\left(\not p_{2} p_{1} R \cdot \bar{\Gamma}_{\mathrm{NA}}\right)
\end{array}\right]} \\
& {\left[\begin{array}{l}
\bar{\Gamma}_{2} \\
\bar{\Gamma}_{3} \\
\bar{\Gamma}_{4} \\
\bar{\Gamma}_{8}
\end{array}\right]=\frac{1}{4 \Delta}\left[\begin{array}{cccc}
p_{1}^{2} p_{2}^{2} & \left(2 p_{1} \cdot p_{2} R \cdot p_{2}-p_{2}^{2} R \cdot p_{1}\right) & -p_{1}^{2} R \cdot p_{2} & -p_{1} \cdot p_{2} \\
\left(2 p_{1} \cdot p_{2} R \cdot p_{2}-p_{2}^{2} R \cdot p_{1}\right) & p_{2}^{2} & -p_{1} \cdot p_{2} & -R \cdot p_{2} \\
-p_{1}^{2} R \cdot p_{2} & -p_{1} \cdot p_{2} & p_{1}^{2} & R \cdot p_{1} \\
-p_{1} \cdot p_{2} & -R \cdot p_{2} & R \cdot p_{1} & 1
\end{array}\right]\left[\begin{array}{c}
\operatorname{Tr}\left(R R \cdot \bar{\Gamma}_{\mathrm{NA}}\right) \\
\operatorname{Tr}\left(\not p_{1} R \cdot \bar{\Gamma}_{\mathrm{NA}}\right) \\
\operatorname{Tr}\left(\not p_{2} R \cdot \bar{\Gamma}_{\mathrm{NA}}\right) \\
\operatorname{Tr}\left(R \not p_{2} p_{1} R \cdot \bar{\Gamma}_{\mathrm{NA}}\right)
\end{array}\right]}
\end{aligned}
$$

with

$$
\Delta=p_{1}^{2} p_{2}^{2}-\left(p_{1} \cdot p_{2}\right)^{2}+2 p_{1} \cdot p_{2} R \cdot p_{1} R \cdot p_{2}-p_{1}^{2}\left(R \cdot p_{2}\right)^{2}-p_{2}^{2}\left(R \cdot p_{1}\right)^{2} .
$$

In case the vector $R$ is in the $p_{1}, p_{2}$ plane, the system degenerates. Then the expansion

$$
R \cdot \bar{\Gamma}=\bar{\Gamma}_{1} \mathbf{1}+\bar{\Gamma}_{3} \not p_{1}+\bar{\Gamma}_{4} \not p_{2}+\bar{\Gamma}_{7} \not p_{1} \not p_{2}
$$

suffices, and the components $\bar{\Gamma}_{i}$ can be read off

$$
\begin{aligned}
& {\left[\begin{array}{l}
\bar{\Gamma}_{1} \\
\bar{\Gamma}_{7}
\end{array}\right]=\frac{1}{4\left(p_{1}^{2} p_{2}^{2}-\left(p_{1} \cdot p_{2}\right)^{2}\right)}\left[\begin{array}{cc}
p_{1}^{2} p_{2}^{2} & -p_{1} \cdot p_{2} \\
-p_{1} \cdot p_{2} & 1
\end{array}\right]\left[\begin{array}{c}
\operatorname{Tr}\left(R \cdot \bar{\Gamma}_{\mathrm{NA}}\right) \\
\operatorname{Tr}\left(\not p_{2} \not p_{1} R \cdot \bar{\Gamma}_{\mathrm{NA}}\right)
\end{array}\right]} \\
& {\left[\begin{array}{l}
\bar{\Gamma}_{3} \\
\bar{\Gamma}_{4}
\end{array}\right]=\frac{1}{4\left(p_{1}^{2} p_{2}^{2}-\left(p_{1} \cdot p_{2}\right)^{2}\right)}\left[\begin{array}{cc}
p_{2}^{2} & -p_{1} \cdot p_{2} \\
-p_{1} \cdot p_{2} & p_{1}^{2}
\end{array}\right]\left[\begin{array}{c}
\operatorname{Tr}\left(\not p_{1} R \cdot \bar{\Gamma}_{\mathrm{NA}}\right) \\
\operatorname{Tr}\left(\not p_{2} R \cdot \bar{\Gamma}_{\mathrm{NA}}\right)
\end{array}\right]}
\end{aligned}
$$

If one chooses first a vector along the 2 (or 3 ) axis, and employs the full projection above, the combinations $\Gamma_{3}, \Gamma_{8}, \Gamma_{9}, \Gamma_{12}$ can be separated. Then choosing $R$ along the fourth axis picks up in addition the $p_{2}^{\mu}$ contributions, and projecting now

$$
R \cdot \bar{\Gamma}=\Gamma^{4}-\Gamma_{3} \gamma^{4}-\Gamma_{8} \not p_{1} \gamma^{4}-\Gamma_{9} \not p_{2} \gamma^{4}-\Gamma_{12} \not p_{1} \not p_{2} \gamma^{4}
$$

we can reconstruct $\Gamma_{2}, \Gamma_{5}, \Gamma_{7}, \Gamma_{11}$. Once these are known we can calculate along the first axis to pick up the $p_{1}^{\mu}$ contributions extracted from

$$
\begin{aligned}
R \cdot \bar{\Gamma}= & \Gamma^{1}-\Gamma_{2} p_{2}^{1}-\Gamma_{3} \gamma^{1}-\Gamma_{5} \not p_{1} p_{2}^{1}-\Gamma_{7} \not p_{2} p_{2}^{1}-\Gamma_{8} \not p_{1} \gamma^{1}-\Gamma_{9} \not p_{2} \gamma^{1}-\Gamma_{11} \not p_{1} \not p_{2} p_{2}^{1} \\
& -\Gamma_{12} \not p_{1} \not p_{2} \gamma^{1}
\end{aligned}
$$

which finally allows to calculate the remaining $\Gamma_{1}, \Gamma_{4}, \Gamma_{6}, \Gamma_{10}$.

\section{F.1. Degenerate points $p_{1}=p_{2}$}

The kinematical limit $p_{1}=p_{2}:=p$, which corresponds to the infrared limit of the soft gluon section, cf. A 2, requires special attention. Here $\Delta$ defined in Eq. (F3) vanishes and the linear system becomes ill-defined: this can be tracked to a reduction of the linear space spanned by the vertex, now

$$
\begin{aligned}
& \Gamma=\Gamma_{1}^{\mathrm{sym}} p^{\mu}+\Gamma_{3}^{\mathrm{sym}} \gamma_{\mu}+\Gamma_{4}^{\mathrm{sym}} \not p p^{\mu}+\Gamma_{8}^{\mathrm{sym}} \not p \gamma^{\mu} \\
& R \cdot \bar{\Gamma}=\bar{\Gamma}_{1}^{\mathrm{sym}} \mathbf{I}+\bar{\Gamma}_{2}^{\mathrm{sym}} R+\bar{\Gamma}_{3}^{\mathrm{sym}} \not p+\bar{\Gamma}_{5}^{\mathrm{sym}} \not p R .
\end{aligned}
$$

The general linear system now can be easily solved:

$$
\left[\begin{array}{l}
\bar{\Gamma}_{1}^{\text {sym }} \\
\bar{\Gamma}_{5}^{\text {sym }} \\
\bar{\Gamma}_{2}^{\text {sym }} \\
\bar{\Gamma}_{3}^{\text {sym }}
\end{array}\right]=\frac{1}{4\left(p^{2}-(R \cdot p)^{2}\right)}\left[\begin{array}{cccc}
p^{2} & -R \cdot p & 0 & 0 \\
-R \cdot p & 1 & 0 & 0 \\
0 & 0 & p^{2} & -R \cdot p \\
0 & 0 & -R \cdot p & 1
\end{array}\right]\left[\begin{array}{c}
\operatorname{Tr}(R \cdot \bar{\Gamma}) \\
\operatorname{Tr}(R \not p R \cdot \bar{\Gamma}) \\
\operatorname{Tr}(\not R R \cdot \bar{\Gamma}) \\
\operatorname{Tr}(\not p R \cdot \bar{\Gamma})
\end{array}\right]
$$


To obtain the various projected functions the algorithm is in all similar to the general case. With $p$ along the 1 axis, choose $\mathrm{R}$ along the 2 axis $\left(R \cdot \bar{\Gamma}=\Gamma^{2}\right)$ and solve for the barred $\bar{\Gamma}$ by means of Eq. (F10), then equating

$$
\Gamma_{3}^{\text {sym }}=\bar{\Gamma}_{2}^{\text {sym }} \quad \Gamma_{8}^{\text {sym }}=\bar{\Gamma}_{5}^{\text {sym }} .
$$

Next choose $R$ along the $p$ (first in our kinematics) axis and employ

$$
R \cdot \bar{\Gamma}=\Gamma^{1}-\Gamma_{3}^{\mathrm{sym}} \gamma^{1}-\Gamma_{8}^{\mathrm{sym}} \not p \gamma^{1}
$$

after solving

$$
\bar{\Gamma}_{1}^{\text {sym }}=\frac{1}{4} \operatorname{Tr}(R \cdot \bar{\Gamma}) \quad \bar{\Gamma}_{3}^{\text {sym }}=\frac{1}{4 p^{2}} \operatorname{Tr}(\not p R \cdot \bar{\Gamma})
$$

the last needed terms can be extracted

$$
\Gamma_{1}^{\text {sym }}=\bar{\Gamma}_{1}^{\text {sym }} / p, \quad \Gamma_{4}^{\text {sym }}=\bar{\Gamma}_{3}^{\text {sym }} / p
$$

\section{F.2. Transformation to the Ball-Chiu basis}

If we denote collectively the various $L_{i}$ and $T_{i}$ by $H_{i}$, and the tensors in Dirac space with one index $\mu$ that appear in Eq. (F1) above by $\bar{H}_{i}$ then, since the vertex accepts a decomposition in both basis, we have

$$
\Gamma=h_{i} H_{i}=\Gamma_{i} \bar{H}_{i} .
$$

We wish to obtain the new coefficient functions $h_{i}$ (split into the four WT functions $\lambda_{1} \ldots \lambda_{4}$ and the eight transverse functions $\left.\tau_{1} \ldots \tau_{8}\right)$ in terms of our calculated $\Gamma_{i} i \subset(1,12)$. This is given in terms of a linear transformation (not unitary since neither basis is orthonormal)

$$
h_{i}=A_{i j} \Gamma_{j} .
$$

Observing Eq. (F14) it is obvious that

$$
H_{i}=A_{i j}^{-1 \mathrm{~T}} \bar{H}_{j}
$$

but the matrix $A_{i j}^{-1 \mathrm{~T}}$ is simple to obtain by directly expanding Eq. (A2) and passing $\gamma_{\mu}$ to the right to compare with Eq. (F1). The resulting matrix has two separate blocks, one connecting the terms with an odd number of $\gamma$ matrices between themselves, another with the even terms. Inverting it we immediately find

$$
\begin{aligned}
& {\left[\begin{array}{l}
\lambda_{1} \\
\lambda_{2} \\
\tau_{2} \\
\tau_{3} \\
\tau_{6} \\
\tau_{8}
\end{array}\right]=\frac{1}{4}\left[\begin{array}{cccccc}
4 & -2 p_{1} \cdot p_{3} & -2 p_{2} \cdot p_{3} & 2 p_{1} \cdot p_{3} & 2 p_{2} \cdot p_{3} & 2\left(2 p_{1}^{2}-p_{3}^{2}\right) \\
0 & \frac{-2 p_{1} \cdot p_{3}}{\Delta} & \frac{-2 p_{2} \cdot p_{3}}{\Delta} & \frac{-2 p_{1} \cdot p_{3}}{\Delta} & \frac{-2 p_{2} \cdot p_{3}}{\Delta} & \frac{-2 p_{3}^{2}}{\Delta} \\
0 & \frac{2}{\Delta} & \frac{-2}{\Delta} & \frac{2}{\Delta} & \frac{-2}{\Delta} & \frac{-4}{\Delta} \\
0 & 1 & -1 & -1 & 1 & 0 \\
0 & -1 & -1 & 1 & 1 & -2 \\
0 & 0 & 0 & 0 & 0 & 4
\end{array}\right]\left[\begin{array}{c}
\Gamma_{3} \\
\Gamma_{4} \\
\Gamma_{5} \\
\Gamma_{6} \\
\Gamma_{7} \\
\Gamma_{12}
\end{array}\right]} \\
& {\left[\begin{array}{l}
\lambda_{3} \\
\lambda_{4} \\
\tau_{1} \\
\tau_{4} \\
\tau_{5} \\
\tau_{7}
\end{array}\right]=\frac{1}{4}\left[\begin{array}{cccccc}
\frac{4 p_{1} \cdot p_{3}}{\Delta} & \frac{4 p_{2} \cdot p_{3}}{\Delta} & \frac{4 p_{1} \cdot p_{3}}{\Delta} & \frac{4 p_{2} \cdot p_{3}}{\Delta} & \frac{4 p_{1} \cdot p_{2} p_{1} \cdot p_{3}}{\Delta} & \frac{4 p_{1} \cdot p_{2} p_{2} \cdot p_{3}}{\Delta} \\
0 & 0 & -2 & -2 & -2 p_{1} \cdot p_{3} & -2 p_{2} \cdot p_{3} \\
\frac{-4}{\Delta} & \frac{4}{\Delta} & \frac{-4}{\Delta} & \frac{4}{\Delta} & \frac{-4 p_{1} \cdot p_{2}}{\Delta} & \frac{4 p_{1} \cdot p_{2}}{\Delta} \\
0 & 0 & 0 & 0 & -1 & 1 \\
0 & 0 & -2 & 2 & 0 & 0 \\
0 & 0 & 0 & 0 & -2 & -2
\end{array}\right]\left[\begin{array}{c}
\Gamma_{1} \\
\Gamma_{2} \\
\Gamma_{8} \\
\Gamma_{9} \\
\Gamma_{10} \\
\Gamma_{11}
\end{array}\right]}
\end{aligned}
$$

with $\Delta=\left(p_{2}^{2}-p_{1}^{2}\right)$. We have to keep in mind that there is a singular kinematical limit. When $p_{1}=p_{2}:=p$ the vertex expansion in the naive basis Eq. (F1) degenerates to 


$$
\begin{aligned}
& \left(\Gamma_{1}+\Gamma_{2}+p^{2}\left(\Gamma_{10}+\Gamma_{11}\right)\right) p^{\mu}+\left(\Gamma_{3}+p^{2} \Gamma_{12}\right) \gamma^{\mu}+\left(\Gamma_{4}+\Gamma_{5}+\Gamma_{6}+\Gamma_{7}\right) \not p p^{\mu}+\left(\Gamma_{8}\right. \\
& \left.\quad+\Gamma_{9}\right) \not p \gamma^{\mu}
\end{aligned}
$$

and since all the transverse tensors vanish $T_{i}(p 1=p 2)=0$ the decomposition in the basis of Eq. (A2) referred to the naive basis in Eq. (F8) reduces to

$$
\left[\begin{array}{l}
\lambda_{1} \\
\lambda_{2} \\
\lambda_{3} \\
\lambda_{4}
\end{array}\right]=\left[\begin{array}{cccc}
1 & 0 & 0 & 0 \\
0 & -\frac{1}{4} & 0 & 0 \\
0 & 0 & \frac{\mathrm{i}}{2} & \frac{\mathrm{i}}{2} \\
0 & 0 & 0 & \frac{-\mathrm{i}}{2}
\end{array}\right]\left[\begin{array}{c}
\Gamma_{3}^{\mathrm{sym}} \\
\Gamma_{4}^{\mathrm{sym}} \\
\Gamma_{1}^{\mathrm{sym}} \\
\Gamma_{8}^{\mathrm{sym}}
\end{array}\right] .
$$

\section{Appendix G. Diagrammatic construction of the quark-quark scattering kernel}

While one does not need to be particularly careful when constructing a truncation of the heavyquark-heavy-quark scattering kernel, the chiral Ward identities need to be respected if one is to extend such model to light quarks. This implies an intimate relation between the scattering kernel and the gap equation and forbids the naive skeleton expansion used in the subsection 5.2.

A systematic recipe to construct a scattering kernel that is consistent with a given quark propagator, respecting the chiral Ward identities, has been given by Munczek [72]. The basic idea is to take functional derivatives of the quark self-energy with respect to the quark propagator. This procedure can be iterated for any type of semi-perturbative or diagrammatic construction of the quark-gluon vertex by cutting all additional quark lines in the contributing diagrams.

In the most general case, i.e., with an arbitrary vertex, Munczek's construction would entail cutting the self-energy diagrams

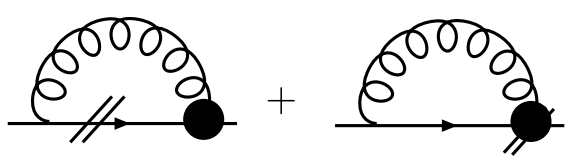

This would naturally lead to a Dyson-Schwinger equation for the connected quark-quark scattering kernel

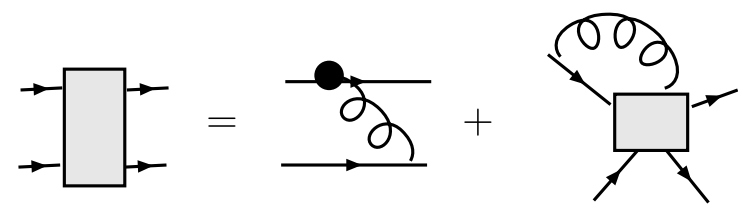

that couples the four-point quark scattering kernel to a higher five-point function. This has been recently and independently stressed by Matevosyan, Tandy and Thomas [41]. However, the confining properties of this kernel are not apparent. Indeed if we analyze the scaling of the first term on the right hand side as a function of the gluon momentum we obtain kernel $\left(k^{2}\right) \sim\left(k^{2}\right)^{-1 / 2-\kappa}\left(k^{2}\right)^{2 \kappa-1} \sim\left(k^{2}\right)^{\kappa-3 / 2}$ which is not singular enough to represent a confining potential. If, however, we would have two dressed quark-gluon vertices in this diagram we obtain kernel $\left(k^{2}\right) \sim\left(k^{2}\right)^{-1-2 \kappa}\left(k^{2}\right)^{2 \kappa-1} \sim\left(k^{2}\right)^{-2}$, leading to a linear rising potential as discussed in Section 5.2 .

In order to make this behavior of the kernel explicit we propose the following scheme: (1) we employ a skeleton expansion for the (partially) 2PI quark-gluon scattering kernel to 
obtain a self-consistent equation for the quark-gluon vertex which reproduces our results from the main part of this paper, (2) we employ a symmetric formulation of the quark self-energy to obtain Munczek's quark-quark scattering kernel with appropriate propagator cuts. This symmetric formulation will lead to disagreement with perturbation theory, but this can be systematically controlled. Then in step (3) the higher n-point-functions appearing in Munczek's construction can be obtained from a closed system of equations by cutting the system in step (1).

Let us give all these equations in diagrammatic form. Step (1) is materialized by giving the exact equation for the quark-gluon vertex in t-channel formulation, Fig. 4, the BS equation for the connected kernel appearing there, and a model for the 2PI scattering kernel in terms of the quark-gluon vertex:

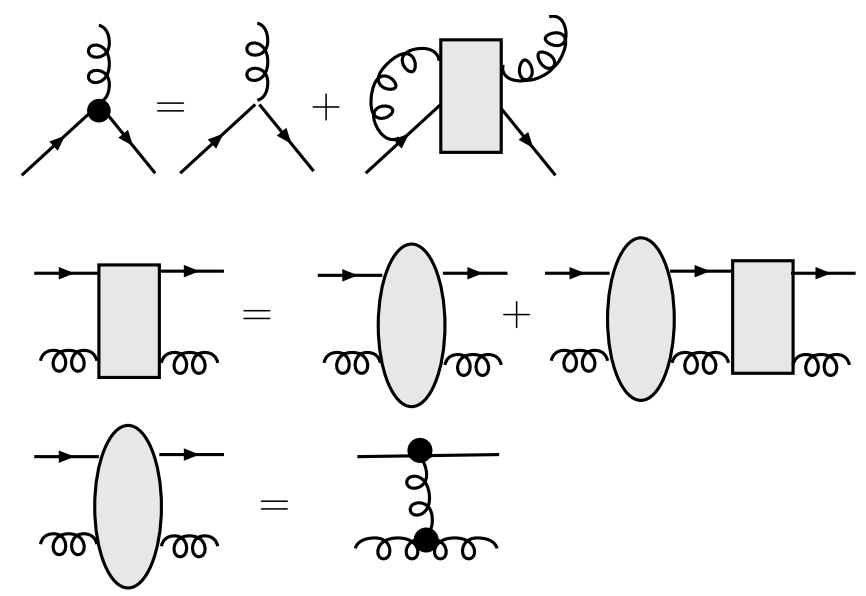

These three equations form a closed system whose input are the dressed quark propagator, which is addressed below, and the dressed gluon propagator and three-gluon vertex. In the quenched approximation considered in this work these functions do not depend on the quark propagator.

For step (2) let us express the self energy in a more symmetric form. By substituting the quarkgluon vertex DSE in t-channel form, Fig. 4, into the self energy one obtains

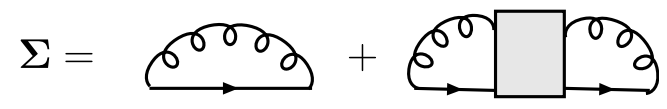

We now cast this self-energy in a form that is amenable to systematic improvement.

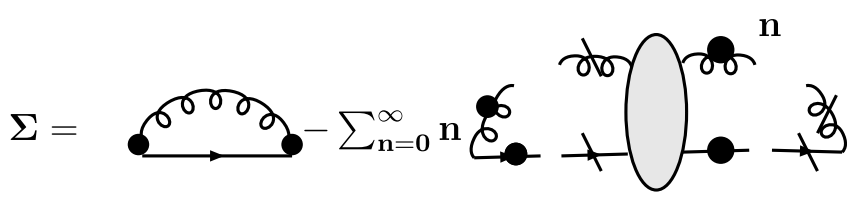

This equation is easily obtained by substituting the DSE for the quark-gluon four-point function, second of Eqs. (G2), into Eq. (G3) above.

Resumming the series in (G4) using $\sum_{n=0}^{\infty} n x^{n}=x\left(\frac{1}{1-x}\right)^{2}$. would bring us back to the asymmetric formulation of the self-energy. In the present form Eq. (G4) is useful since it explicitly contains the confining part of the interaction given by the diagram with two dressed quark-gluon vertices. A truncation of Eq. (G4) at a given $n$ serves as a practical model for a confining kernel which can be worked upon using Munczek's construction. The obvious approximation is to keep the first term with two dressed vertices and discard all terms in the sum. The resulting self-energy disagrees with perturbation theory at the two-loop level. But this is no worse than the often employed approximation of keeping the self-energy formally exact but approximating the quark-gluon vertex at one-loop in the skeleton expansion as in the third part of Eq. (G2). Moreover, the inclusion of terms with a higher 
$n$ iterations of the 2PI kernel systematically improves the perturbative properties of Eq. (G4). Note that also the 2PI kernel was approximated at first order in the skeleton expansion, so it also disagrees with perturbation theory at one loop (two-loops in the self-energy). This also needs to be systematically improved with the help of the skeleton expansion to finally match perturbation theory at any given order.

Cutting the self-energy equation then yields an equation for the quark-quark scattering kernel:

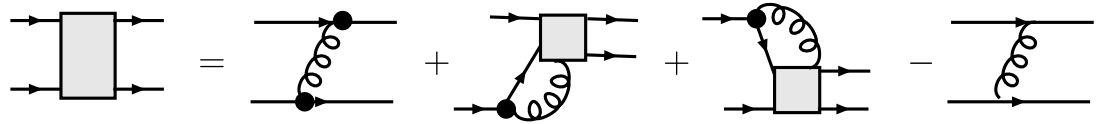

where we have used the derivative of the vertex with respect to the propagator yielding a five-point function. To obtain an equation for this five-point function we take a derivative of Eq. (G2) with respect to the quark propagator, yielding
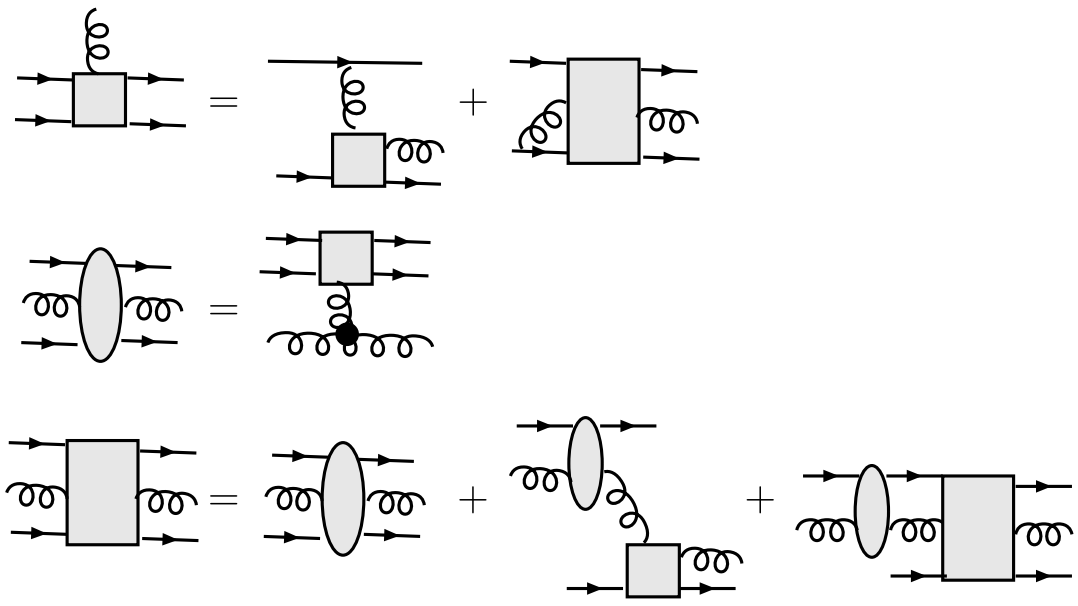

It is thus apparent that we have achieved a truncation that is both chirally symmetric and confining. The quark propagator can be obtained from the first term in Eq. (G4) with quark and gluon vertex given by simultaneously solving the two-equation system (G2). The matching quark scattering kernel is then obtained from Eq. (G5) with the attending five-point function given by the two equations in (G6). One has two systems of two equations that need to be solved sequentially.

It is also apparent how the truncation can be matched to perturbation theory to any given order in a systematic way by expanding a geometric series of 2PI quark-gluon scattering kernels, and expanding the 2PI kernel itself. The number of terms as usual grows very rapidly (but also in perturbation theory). This is a hard task, and it is far from our intention to develop this program in the near future, but at least we have found an algorithm for the wanted construction that may allow statements of principle.

Alternatively, and especially for heavy quark applications, one is not so interested in preserving chiral symmetry explicitly, since it is already badly broken by the large current quark mass. Then a simple skeleton truncation of the kernel may represent a suitable approximation.

\section{Appendix H. Coupling to photons}

In this appendix let us comment on the quark-photon vertex. Hard electromagnetic probes (Deep Inelastic Scattering off the nucleon, jets in electron-positron collisions) and final states (Drell-Yan processes) are the tell-tale experiments about the existence of quarks. Moreover, electromagnetic form factors also provide a glimpse of hadron structure at intermediate energies. It is therefore worth paying a moment's attention to the quark-photon coupling to see what effect if any does the infrared counting have on it. 
The Bethe-Salpeter equation for the quark-photon coupling to flavor $i$, that for $N_{f}$ active flavors, and in terms of the $j \rightarrow i$ (possibly flavor changing) scattering kernels reads

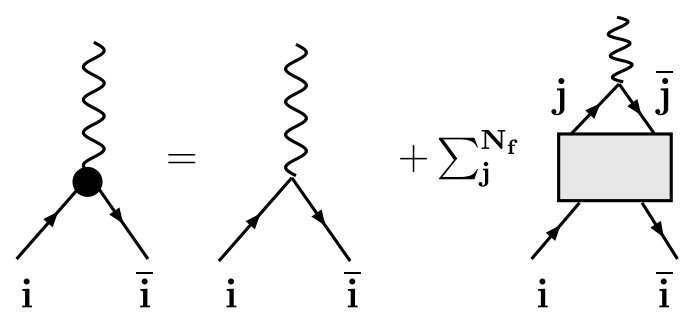

The first term is irrelevant for the purpose of infrared counting. The second displays the quarkquark scattering kernels, that have an infrared dimension of $\left(p^{2}\right)^{-2}$ This is compensated by the integration measure $d^{4} p$, so that the infrared counting becomes trivial. Thus, the equation is consistent with an infrared-finite solution. This is also what one would expect from the Abelian Ward identity and vector meson dominance. In addition, past and recent phenomenological studies of various form factors [111-114] seem to do well without the need for an infrared enhanced vertex.

A further question is whether any experiment so far is actually sensitive to the infrared divergences. We cannot think of an observable that really constrains this. For example, if one thinks of time-like pair production or jets at $e^{-} e^{+}$colliders, the diagram that would show our enhanced quark-gluon vertex is

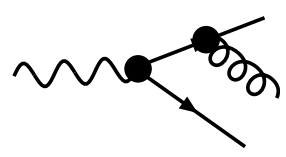

One sees immediately that, by the very definition of a jet, the soft-gluon kinematic point is cut out of the data.

It does appear that one cannot constrain the infrared counting easily from experiment. A first attempt in this direction is given in Ref. [115], but much more work is necessary. Similar considerations apply to the coupling of quarks to other external currents, such as axial currents necessary for the weak interactions of hadrons.

\section{Appendix I. The Gross-Yennie argument and its failure in QCD}

\section{I.1. Crossed ladders simplify $k_{0}$ pole analysis in $Q E D$}

In the following we explain why the familiar Gross-Yennie construction of a potential for photon exchange in QED cannot be applied to gluon exchange within QCD.

In an equal-time description, all physical particles are put on-shell and energy is not conserved in the interaction vertices. Therefore, we start by considering the first skeleton diagram of our (heavy) quarkquark scattering kernel in the center of momentum (CoM) frame, with total energy $W=P^{0}$ as customary,

$$
\begin{array}{lll}
\left(\frac{\mathbf{W}}{\mathbf{2}}+\mathbf{q}^{\mathbf{0}}, \mathbf{q}\right) & \left(\frac{\mathbf{W}}{\mathbf{2}}+\mathbf{q}^{\mathbf{0}^{\prime}}, \mathbf{q}^{\prime}\right) \\
\left(\frac{\mathbf{W}}{\mathbf{2}}-\mathbf{q}^{\mathbf{0}},-\mathbf{q}\right) \longrightarrow & \left(\frac{\mathbf{W}}{\mathbf{2}}-\mathbf{q}^{\mathbf{0}^{\prime}},-\mathbf{q}^{\prime}\right)
\end{array}
$$


and apply our IR counting. In Euclidean space, if all scales were simultaneously sent to zero one obtains $\left(P_{E}^{2}\right)^{2(-1 / 2-\kappa)-1+2 \kappa}=\frac{1}{P_{E}^{4}}$, i.e., a strong IR kernel in terms of the Euclidean momentum $P_{E}$. With $\left(\frac{W}{2}+q_{0}\right)$ (and all others) fixed at its on-shell value by a Dirac delta function (see also below), all other singularities in the $\left(\frac{W}{2}+q_{0}\right)$ plane cancel and one can perform a Wick rotation. The statement is then that if all particles were to go on shell simultaneously, the skeleton diagram would be proportional to the inverse fourth-power of the off-shell momentum $P$. However, to write down a Hamiltonian description we only put the four quarks on shell, the gluon rung giving rise to a potential interaction. Therefore, the condition that the gluon also goes on shell for our vertex becoming strongly enhanced,

$$
\left(q^{0}-q^{0^{\prime}}\right)^{2}-\left(\mathbf{q}-\mathbf{q}^{\prime}\right)^{2} \rightarrow 0
$$

becomes, taking into account the mass-shell condition for the four quark legs that has been imposed beforehand,

$$
\left(\frac{W}{2}-q_{0}\right)^{2}-\mathbf{q}^{2}=M^{2}
$$

(and three others),

$$
\left(E_{q}-E_{q^{\prime}}\right)^{2}-\left(\mathbf{q}-\mathbf{q}^{\prime}\right)^{2} \rightarrow 0
$$

which therefore leads to the statement

$$
\widehat{V}\left(\mathbf{q}-\mathbf{q}^{\prime}\right)=\frac{8 \pi \sigma_{1}}{\left(\mathbf{q}-\mathbf{q}^{\prime}\right)^{4}}
$$

that is, a strong confining potential with string tension named $\sigma_{1}$ (for the first term in the skeleton expansion) and Fourier transform to coordinate space linear in the relative coordinate

$$
V(r)=\sigma_{1} r .
$$

Here $\sigma_{1}$ includes the Casimir color factor $4 / 3$.

The string tension can be extracted from our kernel by contracting all its indices with appropriate spinors. One can of course not trust the Born approximation since the kernel is strong, and therefore an iteration mandatory.

Upon iteration of the kernel (following diagram on the left), the quarks in the intermediate state are now integrated over the off-mass shell region

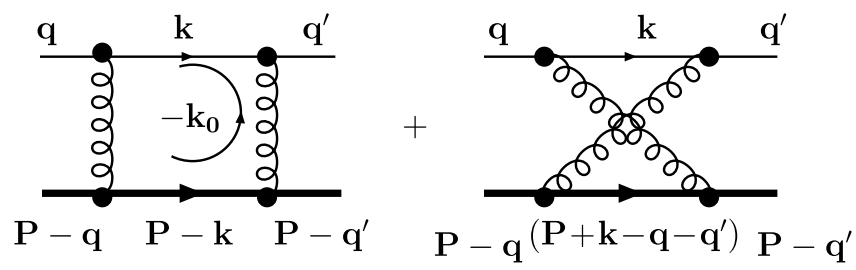

This equation is strictly valid only in QED, with fermions interacting via photon exchange. There, it has been shown by Gross and Yennie how the addition of the crossed-rung diagram leads to the correct one-body limit (the second particle moves in a static potential created by the first particle whose mass is large). To see this, assume that the thicker bottom line in the diagrams above corresponds to a heavy particle of mass $M \rightarrow \infty$. Then the two propagators of the heavy particle in both diagrams can be combined. In the static limit, the physical intuition is that the total energy becomes the energy of the lighter particle plus the mass of the heavy particle that can absorb recoil momentum without absorbing energy, $P_{0}=e+M$, with $e=q_{0}=q_{0}^{\prime}$. For the heavy particle we will also ignore the negative energy part of the propaga- 
tor, suppressed by a further $1 / \mathrm{M}$. With this notation, the difference of the two diagrams is given by a common factor times the difference of the internal fermion propagators in the heavy mass limit, i.e.,

$$
\begin{aligned}
& \frac{1}{M^{2}-(P-k)^{2}-\mathrm{i} \epsilon}+\frac{1}{M^{2}-\left(P+k-q-q^{\prime}\right)^{2}-\mathrm{i} \epsilon} \\
& \rightarrow \frac{1}{2 M}\left(\frac{1}{M-P_{0}+k_{0}-\mathrm{i} \epsilon}+\frac{1}{M-P_{0}-k_{0}+q_{0}+q_{0}^{\prime}-\mathrm{i} \epsilon}\right) \\
& =\frac{1}{2 M}\left(\frac{1}{k_{0}-e-\mathrm{i} \epsilon}-\frac{1}{k_{0}-e+\mathrm{i} \epsilon}\right)=\frac{2 \pi \mathrm{i} \delta\left(k_{0}-e\right)}{2 M} .
\end{aligned}
$$

Thus, if one of the particle's masses is sent to infinity, the intermediate $k_{0}$ variables are fixed to their on-shell value $e$ as intuitively expected. The Wick rotation is then possible, and one can take the large $M$ limit for the second particle if so wished. Gross and Yennie have given the induction step leading to the cancellation to all orders of perturbation theory [116], by summing all ladders and crossed ladders. The argument is valid in the presence of vertex and gluon-propagator dressing functions, if the naive vertex mass-counting of perturbation theory holds, and thus their proof is essentially complete for QED in the static (automatically quenched) limit.

In QCD, however, there appear extra color factors in each of the diagrams, such that the simple cancellation mechanism of Gross and Yennie cannot work. We are aware of no extension of the argument to include color factors as well as QCD gluon radiation. The need for including all crossed ladders makes difficult to show the cancellation explicitly when one rewrites the perturbation series as an integral equation,
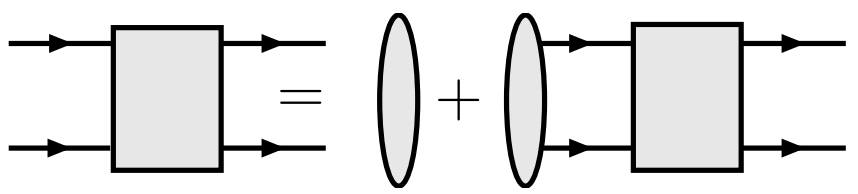

as the canceling poles are entangled between the explicit propagators and the 2PI kernel. Therefore, no truncation of the full Bethe-Salpeter equation is known that consistently takes the one-body limit correctly.

\section{I.2. The Gross equation}

Given that the Salpeter equation (see Section 7.2 in the main part of this paper) needs an inconsistent approximation of the energy $k_{0}$ thus introducing an uncontrollable systematic error, Gross devised an equation inspired by the Gross-Yennie cancellation mechanism. The idea is to replace the bona-fide propagators $S_{q} S_{\bar{q}}$ from the field theory by an ad-hoc Gross function, containing a Dirac delta that puts the quarks on their mass-shell, and subsequently evaluating the kernel at the pole thus forced. The Gross function in the center of mass frame for undressed fermions is simple and natural,

$$
G(k, P)=\frac{2 \pi \delta\left(\left(S_{q}^{-1}-S_{\bar{q}}^{-1}\right) / 2\right)}{S_{q}^{-1}+S_{\bar{q}}^{-1}}=\frac{2 \pi \delta\left(k_{0}\right)}{2 P^{0}\left(M^{2}+\mathbf{k}^{2}-P_{0}^{2} / 4\right)} .
$$

However, in the presence of momentum-dependent dressing functions, one obtains a quite tedious two-particle propagator, where we use the shorthands $A_{ \pm}=A\left(\left(P^{0} / 2 \pm k^{0}\right)^{2}-\mathbf{k}^{2}\right)$ (sim. for $\left.\mathrm{B}\right)$,

$$
\begin{aligned}
& \left(\left(k+\frac{P^{0}}{2} \gamma^{0}\right) A_{+}-B_{+}\right) \times\left(\left(-\not k+\frac{P^{0}}{2} \gamma^{0}\right) A_{-}-B_{-}\right) \delta\left(k^{0}-\bar{k}^{0}\right) \\
& {\left[\left(2\left(P_{0}+2 k_{0}\right)\left(B_{+} B_{+}^{\prime}+\left(\left(P^{0} / 2+k^{0}\right)^{2}-\mathbf{k}^{2}\right) A_{+} A_{+}^{\prime}\right)-2\left(P_{0}-2 k_{0}\right)\left(B_{-} B_{-}^{\prime}\right.\right.\right.} \\
& \left.\left.\left.+\left(\left(P^{0} / 2-k^{0}\right)^{2}-\mathbf{k}^{2}\right) A_{-} A_{-}^{\prime}\right)+\left(P_{0}+2 k_{0}\right) A_{+}^{2}-\left(P_{0}-2 k_{0}\right) A_{-}^{2}\right)\left(\left(\left(P^{0} / 2+k^{0}\right)^{2}-\mathbf{k}^{2}\right) A_{+}^{2}+B_{+}^{2}\right)\right]^{-1}
\end{aligned}
$$


where the on-shell pole $\bar{k}^{0}$ is now located by solving the implicit equation

$$
B_{+}^{2}-B_{-}^{2}+\left(\left(P^{0} / 2+\bar{k}^{0}\right)-\mathbf{k}^{2}\right) A_{+}^{2}-\left(\left(P^{0} / 2-\bar{k}^{0}\right)-\mathbf{k}^{2}\right) A_{-}^{2}=0
$$

(a numeric fixed-point method or Newton iteration is necessary since the $B_{+}$, etc. functions depend on $\bar{k}^{0}$ ). The resulting local potential that would go into Eq. (76) is again formally the same as with the Born or Salpeter constructions, except for the different pole position, possibly causing a shift in the gluon momentum and obscuring the confining soft-gluon divergence, and in addition the resulting non-local kinetic energy for the equation is markedly more difficult.

It is difficult to see how a confining potential arises here except in the limit $A \rightarrow 1, B \rightarrow M$ where $\bar{k}_{0} \rightarrow 0$ reproducing essentially the same result as the one of Section 7.2.

\section{References}

[1] S. Weinberg, Phys. Rev. Lett. 31 (1973) 494; D.J. Gross, F. Wilczek, Phys. Rev. D 8 (1973) 3633.

[2] W.J. Marciano, H. Pagels, Phys. Rep. 36 (1978) 137.

[3] R. Alkofer, C.S. Fischer, F.J. Llanes-Estrada, Phys. Lett. B 611 (2005) 279. Available from: <hep-th/0412330>.

[4] C.S. Fischer, J.M. Pawlowski, Phys. Rev. D 75 (2007) 025012. Available from: <hep-th/0609009>.

[5] L. von Smekal, R. Alkofer, A. Hauck, Phys. Rev. Lett. 79 (1997) 3591. Available from: <hep-ph/9705242>.

[6] L. von Smekal, A. Hauck, R. Alkofer, Ann. Phys. 267 (1998) 1 (Erratum-ibid. 269 (1998) 182). Available from: <arXiv:hep$\mathrm{ph} / 9707327>$.

[7] C. Lerche, L. von Smekal, Phys. Rev. D 65 (2002) 125006. Available from: <hep-ph/0202194>.

[8] J.M. Pawlowski, D.F. Litim, S. Nedelko, L. von Smekal, Phys. Rev. Lett. 93 (2004) 152002. Available from: <hep-th/ 0312324>.

[9] C.S. Fischer, R. Alkofer, Phys. Rev. D 67 (2003) 094020. Available from: <hep-ph/0301094>.

[10] A.I. Davydychev, P. Osland, L. Saks, Phys. Rev. D 63 (2001) 014022. Available from: <hep-ph/0008171>.

[11] J. Skullerud, A. Kizilersu, JHEP 0209 (2002) 013. Available from: <hep-ph/0205318>; J. Skullerud et al, JHEP 0304 (2003) 047. Available from: <hep-ph/0303176>;

A. Kizilersu, D.B. Leinweber, J.I. Skullerud, A.G. Williams, Eur. Phys. J. C 50 (2007) 871. Available from: <hep-lat/0610078>.

[12] H.W. Lin, Phys. Rev. D 73 (2006) 094511. Available from: <hep-lat/0510110>.

[13] F.J. Llanes-Estrada, C.S. Fischer, R. Alkofer, Nucl. Phys. Proc. Suppl. 152 (2006) 43. Available from: <hep-ph/0407332>.

[14] C.S. Fischer, F. Llanes-Estrada, R. Alkofer, Nucl. Phys. Proc. Suppl. 141 (2005) 128. Available from: <hep-ph/0407294>.

[15] M.S. Bhagwat, P.C. Tandy, Phys. Rev. D 70 (2004) 094039. Available from: <hep-ph/0407163>.

[16] M.S. Bhagwat, A. Holl, A. Krassnigg, C.D. Roberts, P.C. Tandy, Phys. Rev. C 70 (2004) 035205. Available from: <nucl-th/ 0403012>.

[17] P. Maris, P.C. Tandy, Nucl. Phys. Proc. Suppl. 161 (2006) 136. Available from: <nucl-th/0511017>.

[18] A.P. Szczepaniak, E.S. Swanson, Phys. Rev. D 55 (1997) 3987. Available from: <hep-ph/9611310>.

[19] P. Bicudo, G. Marques, Phys. Rev. D 70 (2004) 094047. Available from: <hep-ph/0305198>.

[20] P. Bicudo, G.M. Marques, Available from: <hep-ph/0309231> (unpublished).

[21] A.V. Nefediev, Yu.A. Simonov, JETP Lett. 82 (2005) 557 (Pisma Zh. Eksp. Teor. Fiz. 82 (2005) 633). Available from: <hep-ph/ 0510092>.

[22] A.V. Nefediev, Yu.A. Simonov, Available from: <hep-ph/0708.3603>.

[23] R. Alkofer, M.Q. Huber, K. Schwenzer, Available from: <hep-th/0801.2762>.

[24] J.S. Ball, T.W. Chiu, Phys. Rev. D 22 (1980) 2542.

[25] C.D. Roberts, A.G. Williams, Prog. Part. Nucl. Phys. 33 (1994) 477. Available from: <hep-ph/9403224>.

[26] R. Alkofer, L. von Smekal, Phys. Rep. 353 (2001) 281. Available from: <hep-ph/0007355>.

[27] P. Maris, C.D. Roberts, Int. J. Mod. Phys. E 12 (2003) 297. Available from: <nucl-th/0301049>.

[28] C.S. Fischer, J. Phys. G 32 (2006) R253. Available from: <hep-ph/0605173>.

[29] J.M. Pawlowski, Ann. Phys. 322 (2007) 2831. Available from: <hep-th/0512261>.

[30] J. Berges, Phys. Rev. D 70 (2004) 105010. Available from: <hep-ph/0401172>.

[31] J.M. Luttinger, J.C. Ward, Phys. Rev. 118 (1960) 1417.

[32] G. Baym, Phys. Rev. 127 (1962) 1391.

[33] J.M. Cornwall, R. Jackiw, E. Tomboulis, Phys. Rev. D 10 (1974) 2428.

[34] R.E. Norton, J.M. Cornwall, Ann. Phys. 91 (1975) 106.

[35] D.F. Litim, J.M. Pawlowski, Available from: <hep-th/9901063>.

[36] H. Gies, Available from: <hep-ph/0611146>.

[37] C.S. Fischer, H. Gies, JHEP 0410 (2004) 048. Available from: <hep-ph/0408089>.

[38] J. Braun, H. Gies, J.M. Pawlowski, Available from: <hep-th/0708.2413>.

[39] H.J. Lu, C.A.R. Sa de Melo, Phys. Lett. B 273 (1991) 260 (Erratum-ibid. B 285 (1992) 399).

[40] A. Bender, W. Detmold, C.D. Roberts, A.W. Thomas, Phys. Rev. C 65 (2002) 065203. Available from: <nucl-th/0202082>; W. Detmold, Doctoral dissertation submitted to the University of Adelaide.

[41] H.H. Matevosyan, A.W. Thomas, P.C. Tandy, Phys. Rev. C 75 (2007) 045201. Available from: <nucl-th/0605057>.

[42] H.J. Munczek, A.M. Nemirovsky, Phys. Rev. D 28 (1983) 181.

[43] P. Watson, R. Alkofer, Phys. Rev. Lett. 86 (2001) 5239. Available from: <hep-ph/0102332>.

[44] T. Appelquist, J. Carazzone, Phys. Rev. D 11 (1975) 2856.

[45] A. Cucchieri, T. Mendes, Available from: <hep-lat/0710.0412>. 
[46] A. Sternbeck, L. von Smekal, D.B. Leinweber, A.G. Williams, Available from: <hep-lat/0710.1982>.

[47] A.C. Aguilar, D. Binosi, J. Papavassiliou, Available from: <hep-ph/0802.1870>.

[48] Ph. Boucaud, J.P. Leroy, A.L. Yaouanc, J. Micheli, O. Pene, J. Rodriguez-Quintero, Available from: <hep-ph/0801.2721>.

[50] T. Kugo, Available from: <hep-th/9511033>.

[51] J.C. Taylor, Nucl. Phys. B 33 (1971) 436.

[52] D. Zwanziger, Phys. Rev. D 65 (2002) 094039. Available from: <hep-th/0109224>.

[53] R. Alkofer, W. Detmold, C.S. Fischer, P. Maris, Phys. Rev. D 70 (2004) 014014. Available from: <hep-ph/0309077>; R. Alkofer, W. Detmold, C.S. Fischer, P. Maris, Nucl. Phys. Proc. Suppl. 141 (2005) 122. Available from: <hep-ph/0309078>.

[54] R. Alkofer, M.Q. Huber, K. Schwenzer, in preparation.

[55] P.O. Bowman, U.M. Heller, D.B. Leinweber, M.B. Parappilly, A.G. Williams, J.B. Zhang, Phys. Rev. D 71 (2005) 054507. Available from: <hep-lat/0501019>.

[56] C. Anastasiou, E.W.N. Glover, C. Oleari, Nucl. Phys. B 572 (2000) 307. Available from: <hep-ph/9907494>.

[57] R. Alkofer, C.S. Fischer, F.J. Llanes-Estrada, Mod. Phys. Lett. A 23 (2008) 1105. Available from: <hep-ph/0607293>.

[58] W.M. Yao et al., [Particle Data Group], J. Phys. G 33 (2006) $1 .$.

[59] R. Alkofer, P. Bicudo, S.R. Cotanch, C.S. Fischer, F.J. Llanes-Estrada, Available from: <nucl-th/0601032>.

[60] T. Schafer, K. Schwenzer, Phys. Rev. Lett. 97 (2006) 092301. Available from: <hep-ph/0512309>; K. Schwenzer, Nucl. Phys. A 785 (2007) 241.

[61] C. Kellermann, C.S. Fischer, Available from: <hep-ph/0801.2697>.

[62] H. Pagels, Phys. Rev. D 15 (1977) 2991.

[63] L. von Smekal, P.A. Amundsen, R. Alkofer, Nucl. Phys. A 529 (1991) 633.

[64] E. Eichten, F. Feinberg, Phys. Rev. D 10 (1974) 3254.

[65] S.J. Brodsky, Eur. Phys. J. A 31 (2007) 638. Available from: <hep-ph/0610115>.

[66] G. Krein, C.D. Roberts, A.G. Williams, Int. J. Mod. Phys. A 7 (1992) 5607.

[67] R. Oehme, Int. J. Mod. Phys. A 10 (1995) 1995. Available from: <hep-th/9412040>.

[68] C.J. Burden, Phys. Rev. D 57 (1998) 276. Available from: <hep-ph/9702411>; C.J. Burden, Phys. Rev. D 59 (1999) 037502. Available from: <hep-ph/9807438>.

[69] K. Osterwalder, R. Schrader, Commun. Math. Phys. 31 (1973) 83-112; K. Osterwalder, R. Schrader, Commun. Math. Phys. 42 (1975) 281.

[70] U. Habel et al, Z. Phys. A 336 (1990) 423; U. Habel et al, Z. Phys. A 336 (1990) 435; M. Stingl, Z. Phys. A 353 (1996) 423-445. Available from: <hep-th/9502157>.

[71] C. Itzykson, J.B. Zuber, International Series in Pure and Applied Physics, McGraw-Hill, New York, USA, 1980. 705 p.

[72] H.J. Munczek, Phys. Rev. D 52 (1995) 4736. Available from: <hep-th/9411239>.

[73] A. Bender, C.D. Roberts, L. Von Smekal, Phys. Lett. B 380 (1996) 7. Available from: <nucl-th/9602012>.

[74] D. Gromes, Z. Phys. C 11 (1981) 147.

[75] F. Okiharu, H. Suganuma, T.T. Takahashi, Phys. Rev. D 72 (2005) 014505. Available from: <hep-lat/0412012>

[76] F.J. Llanes-Estrada, S.R. Cotanch, P.J.deA. Bicudo, J.E.F. Ribeiro, A.P. Szczepaniak, Nucl. Phys. A 710 (2002) 45. Available from: <hep-ph/0008212>.

[77] J.E.T. Ribeiro, Z. Phys. C 5 (1980) 27.

[78] M.B. Gavela, A. Le Yaouanc, L. Oliver, O. Pene, J.C. Raynal, S. Sood, Phys. Lett. B 82 (1979) 431.

[79] J. Greensite, Prog. Part. Nucl. Phys. 51 (2003) 1. Available from: <hep-lat/0301023>.

[80] R. Alkofer, J. Greensite, J. Phys. G 34 (2007) S3. Available from: <hep-ph/0610365>.

[81] G.S. Bali, Phys. Rev. D 62 (2000) 114503. Available from: <hep-lat/0006022>.

[82] N. Isgur, J.E. Paton, Phys. Rev. D 31 (1985) 2910.

[83] Katja Waidelich, Masters Thesis, North Carolina State University, 2000, Diplomarbeit Univ. Karlsruhe, 2001.

[84] F. Buisseret, C. Semay, Phys. Rev. D 76 (2007) 017501. Available from: <hep-ph/0704.1753>.

[85] G.S. Bali, K. Schilling, A. Wachter, Phys. Rev. D 56 (1997) 2566. Available from: <hep-lat/9703019>.

[86] H. Leutwyler, Phys. Lett. B 98 (1981) 447.

[87] M.B. Voloshin, Nucl. Phys. B 154 (1979) 365.

[88] W.E. Caswell, G.P. Lepage, Phys. Rev. A 18 (1978) 810.

[89] P.J.d. Bicudo, J.E.F. Ribeiro, Phys. Rev. D 42 (1990) 1625.

[90] A. Le Yaouanc, L. Oliver, S. Ono, O. Pene, J.C. Raynal, Phys. Rev. D 31 (1985) 137.

[91] R. Alkofer, P.A. Amundsen, Nucl. Phys. B 306 (1988) 305.

[92] F.J. Llanes-Estrada, S.R. Cotanch, Phys. Rev. Lett. 84 (2000) 1102. Available from: <hep-ph/9906359>.

[93] F.J. Llanes-Estrada, S.R. Cotanch, Nucl. Phys. A 697 (2002) 303. Available from: <hep-ph/0101078>.

[94] R. Alkofer, M. Kloker, A. Krassnigg, R.F. Wagenbrunn, Phys. Rev. Lett. 96 (2006) 022001. Available from: <hep-ph/ 0510028>.

[95] S. Godfrey, N. Isgur, Phys. Rev. D 32 (1985) 189.

[96] J.A.M. Vermaseren, Available from: <math-ph/0010025>.

[97] Y. Koma, M. Koma, H. Wittig, Available from: <hep-lat/0711.2322>.

[98] N. Brambilla, A. Pineda, J. Soto, A. Vairo, Rev. Mod. Phys. 77 (2005) 1423. Available from: <hep-ph/0410047>.

[99] C. Quigg, J.L. Rosner, Comments Nucl. Part. Phys. 8 (1978) 11.

[100] C. Quigg, Available from: <hep-ph/9707493>.

[101] B. Aubert et alBABAR Collaboration, Phys. Rev. Lett. 95 (2005) 142001. Available from: <hep-ex/0506081>.

[102] B. Aubert et alBABAR Collaboration, Phys. Rev. Lett. 98 (2007) 21200. Available from: <hep-ex/0610057>.

[103] Q. He et al., CLEO Collaboration, Phys. Rev. D 74 (2006) 091104. Available from: <hep-ex/0611021>.

[104] X.L. Wang et al., Belle Collaboration, Available from: <hep-ex/0707.3699>.

[105] J.L. Rosner, J. Phys. G 34 (2007) S127. Available from: <hep-ph/0609195>.

[106] A.P. Szczepaniak, E.S. Swanson, Phys. Rev. D 65 (2002) 025012. Available from: <hep-ph/0107078>.

[107] I.J. General, S.R. Cotanch, F.J. Llanes-Estrada, Eur. Phys. J. C 51 (2007) 347. Available from: <hep-ph/0609115>. 
[108] I.J. General, P. Wang, S.R. Cotanch, F.J. Llanes-Estrada, Available from: <hep-ph/0707.1286>.

[109] C.D. Roberts, Available from: <nucl-th/0712.0633>.

[110] C.S. Fischer, R. Alkofer, Phys. Lett. B 536 (2002) 177. Available from: <hep-ph/0202202>.

[111] P. Maris, P.C. Tandy, Phys. Rev. C 62 (2000) 055204. Available from: <nucl-th/0005015>.

[112] M. Oettel, R. Alkofer, L. von Smekal, Eur. Phys. J. A 8 (2000) 553. Available from: <nucl-th/0006082>.

[113] A. Holl, A. Krassnigg, P. Maris, C.D. Roberts, S.V. Wright, Phys. Rev. C 71 (2005) 065204. Available from: <nucl-th/ 0503043>.

[114] M.S. Bhagwat, P. Maris, Available from: <nucl-th/0612069>.

[115] A.C. Aguilar, A. Mihara, A.A. Natale, Int. J. Mod. Phys. A 19 (2004) 249.

[116] F. Gross, Phys. Rev. C 26 (1982) 2203. 